\title{
Tracing cosmic evolution with clusters of galaxies
}

\author{
G. Mark Voit \\ Department of Physics and Astronomy, Michigan State University, East Lansing, MI 48824
}

(Dated: September 10, 2004)

\begin{abstract}
The most successful cosmological models to date envision structure formation as a hierarchical process in which gravity is constantly drawing lumps of matter together to form increasingly larger structures. Clusters of galaxies currently sit atop this hierarchy as the largest objects that have had time to collapse under the influence of their own gravity. Thus, their appearance on the cosmic scene is also relatively recent. Two features of clusters make them uniquely useful tracers of cosmic evolution. First, clusters are the biggest things whose masses we can reliably measure because they are the largest objects to have undergone gravitational relaxation and entered into virial equilibrium. Mass measurements of nearby clusters can therefore be used to determine the amount of structure in the universe on scales of $10^{14}-10^{15} M_{\odot}$, and comparisons of the present-day cluster mass distribution with the mass distribution at earlier times can be used to measure the rate of structure formation, placing important constraints on cosmological models. Second, clusters are essentially "closed boxes" that retain all their gaseous matter, despite the enormous energy input associated with supernovae and active galactic nuclei, because the gravitational potential wells of clusters are so deep. The baryonic component of clusters therefore contains a wealth of information about the processes associated with galaxy formation, including the efficiency with which baryons are converted into stars and the effects of the resulting feedback processes on galaxy formation. This article reviews our theoretical understanding of both the dark-matter component and the baryonic component of clusters, providing a context for interpreting the flood of new cluster observations that are now arriving from the latest generation of X-ray observatories, large optical surveys, and measurements of cluster-induced distortions in the spectrum of the cosmic microwave background.
\end{abstract}

\section{Contents}

\section{Introduction \\ II. Observable Properties of Clusters \\ A. Clusters in Optical Light \\ 1. Optical Richness \\ 2. Galaxv Velocities \\ 3. Gravitational Lensing \\ B. Clusters in X-ravs \\ 1. X-rav Surface Brightness \\ 2. Plasma Temperature \\ 3. Measuring Abundances \\ C. Clusters in Microwaves \\ 1. The S-Z Effect \\ 2. Comparing $\mathrm{S}-\mathrm{Z}$ with $\mathrm{X}-\mathrm{rav}$}

III. Evolution of the Dark Component

A. A Recipe for the Universe

1. Global Dynamics

2. Global Geometry

3. Densitv Perturbations

4. Growth of Linear Perturbations

5. The CDM Power Spectrum

6. Power Spectrum Normalization

7. Summarv of Cosmological Parameters

B. Cluster Formation

1. Spherical Collapse

2. Cluster Mass Profiles

3. Defining Cluster Mass

4. Cluster Mass Function

5. Cluster Bias

C. Measuring the Cluster Mass Function

1. Linking Mass with Observables

2. Mass-Temperature Relation

3. Mass-Luminosity Relation

4. Mass-Richness Relation

5. Velocitv Dispersion and Mass
3

5

6

6

7

10

10

10

11

12

13

14

15

16

16

18

18

19

19

20

22

23

23

24

25

26

27
6. Weak Lensing and Mass

7. Baryons and Mass

D. Evolution of the Mass Function

1. Dependence on Cosmologv

2. Evolution of the Observables

3. Constraints on Dark Matten

4. Constraints on Dark Energy

IV. Evolution of the Baryonic Component

A. Structure Formation and Gravitational Heating

1. Intracluster Entropv

2. Entropy Generation by Smooth Accretion

3. Entropy Generation by Hierarchical Merging

4. Observed Entropv Profiles

B. Galaxv Formation and Feedback

1. Preheating

2. Radiative Cooling

3. Feedback from Supernovae

4. Feedback from Active Galactic Nuclei

5. Transport Processes

C. Galaxy Formation and Cluster Observables

1. Role of Cooling

2. Role of Feedback

3. Role of Smoothing

4. Predictions for Evolution

D. Constraints on Barvon Condensation

1. Mass and Light in Clusters

2. Intergalactic Stars

3. Global S-Z Effect

4. Cooling Flows in Clusters

Acknowledgments 


\section{INTRODUCTION}

Cosmology has recently reached an important milestone. A wide variety of cosmological observations now support a single model for the overall architecture of the observable universe and the development of galaxies and other structures within it. According to this so-called concordance model, the geometry of the observable universe is indistinguishable from a flat geometry, implying that its total energy density is very close to the critical density needed to close the universe. The two dominant components of the universe appear to be a non-baryonic form of dark matter, whose gravity is responsible for structure formation, and a mysterious form of dark energy, whose pressure is currently causing the expansion of the universe to accelerate. The mean density of baryonic matter is about $15 \%$ of the total amount of matter, and we can observe the baryonic matter only because the gravitational attraction of non-baryonic dark matter has drawn the baryonic gas into deep potential wells, where a small fraction of it condenses into stars and galaxies.

This model explains many different features of the observable universe, but it is not entirely satisfying because the nature of the dark matter and the provenance of the dark energy remain unknown. The implications of dark energy for fundamental physics are particularly serious, so we need to be sure that it is absolutely necessary to explain the astronomical observations. In addition, many aspects of galaxy formation remain poorly understood. Dark-matter models successfully account for the spatial distribution of mass in the universe, as traced by the galaxies, but they do not explain all the properties of the galaxies themselves. Dark matter initiates the process of galaxy formation, but once stars begin to form, supernova explosions and disturbances wrought by supermassive black holes can inhibit further star formation by pumping thermal energy into the universe's baryonic gas.

Clusters of galaxies are a particularly rich source of information about the underlying cosmological model, making possible a number of critical tests. According to the concordance model, clusters are the largest and most recent gravitationally-relaxed objects to form because structure grows hierarchically. The universe begins in a state of rapid expansion whose current manifestation is Hubble's Law relating a galaxy's recessional velocity $v_{r}$ to its distance $d$ through Hubble's constant $H_{0}$ : $v_{r}=H_{0} d$. Generalizing this feature of the local universe to all of observable space links an object's cosmological redshift $z=\left(\lambda_{\text {observed }} / \lambda_{\text {rest }}\right)-1$ with a unique time $t(z)$ since the Big Bang, enabling us to probe the evolution of the universe with observations of distant objects. ${ }^{1}$ Grav-

\footnotetext{
1 In this definition, $\lambda_{\text {rest }}$ is the wavelength of a photon emitted by a distant object and $\lambda_{\text {observed }}$ is the wavelength it is observed to have when it reaches Earth.
}

ity drives structure formation in this expanding realm because the matter density is nearly equal to the critical density during much of cosmic history. Regions whose density slightly exceeds the mean density are therefore gravitationally bound and eventually decouple from the expansion, collapse upon themselves, and enter a state of virial equilibrium in which the mean speeds of the component particles are approximately half the escape velocity. Because density perturbations in the concordance model have greater amplitudes on smaller length scales, small sub-galactic objects are the first to decouple, collapse, and virialize. These small objects then collect into galaxies, and galaxies later collect into clusters of galaxies, whose masses now top out at roughly $10^{15}$ times that of the Sun's $\left(10^{15} M_{\odot}\right)$. Thus, the growth and development of clusters directly traces the process of structure formation in the universe.

Section II outlines the observable properties of galaxy clusters that enable us to measure their masses. Observables in the optical band include the overall luminosity of a cluster's galaxies, which scales with the overall mass, the velocity dispersion of a cluster's member galaxies, which responds to the depth of the cluster's potential well, and gravitational lensing of background galaxies by the cluster's potential. Observables in the X-ray band include the overall X-ray luminosity of a cluster, coming from the hot gas trapped in the cluster's gravitational potential, the temperature inferred from the X-ray spectrum of that gas, and the abundances of various elements inferred from the emission lines in that spectrum. This hot gas also leaves an imprint on the microwave sky because its electrons Compton scatter the photons of the cosmic microwave background radiation. Microwave observations are therefore an alternative source of information about the hot gas and its temperature.

Once we have measured the masses of a sample of clusters, we can use that sample to study cosmology. Section III explains how the characteristics of the cluster population relate to cosmological models. It begins by summarizing the elements of the concordance model and provides a number of useful analytical approximations to the results of numerical simulations of cluster formation based on this model. Then it covers the dicey middle ground linking those simulations with observations, currently the main source of uncertainty in deriving cosmological parameters from cluster observations. The section concludes with a look at the evolution observed in the cluster population and the constraints that cluster evolution places on cosmological models.

Section IV takes up the subject of the baryonic component of clusters, with two purposes in mind. First, in order to improve the precision of cosmological measurements with clusters, we need to know how the process of galaxy formation affects the relations used to derive cluster masses from observations of a cluster's hot gas and galaxies. Current numerical simulations accurately reproduce the behavior of the dark component, whose interactions are purely gravitational, but fail to reproduce 
with similar accuracy the observed behavior of the baryonic component, whose interactions are also hydrodynamical and thermodynamical. These discrepancies between simulations and observations indicate that galaxy formation alters the state of a cluster's hot gas in a way that preserves information about the poorly understood feedback processes that regulated galaxy formation long before the cluster reached its present state. Our second purpose is therefore to try to decipher what the state of the hot gas is saying about the process of galaxy formation, so as to gain insight into those feedback processes. Section $\nabla$ concludes the review with some brief remarks about ongoing and future cluster surveys.

Despite this article's length, it falls somewhat short of being a comprehensive review of cluster physics, which would require more pages than this journal is inclined to provide. Instead, I have tried to assemble a readable introduction to cluster evolution for non-experts, concentrating on the middle ground connecting theory to observations and distilling the key theoretical results into a set of simple analytical tools useful to observers. For more on the subject of clusters and their evolution, readers should consult Sarazin (1988), Borgani et al. (2002b), Rosati et al. (2002), and Mulchaev et al. (2004).

\section{OBSERVABLE PROPERTIES OF CLUSTERS}

Clusters of galaxies might have been called something different if they had first been discovered in a waveband other than visible light, because all of the stars in all of a cluster's galaxies represent only a small fraction of a cluster's overall mass. Clusters contain substantially more mass in the form of hot gas, observable with Xray and microwave instruments. This section outlines how clusters are observed in all three of these wavebands and how those observations reveal a cluster's total mass, which turns out to be about seven times the combined baryonic mass in stars and hot gas (Allen et al., 2002; David et al., 1995; Evrard, 1997; White et al., 1993).

\section{A. Clusters in Optical Light}

Optical identification of galaxy clusters has been going on for quite a long time. By the end of the eighteenth century Charles Messier (1784) and William Herschel (1785) had already recognized concentrations of galaxies in the constellations Virgo and Coma Berenices. Today these clusters of galaxies are known as the Virgo cluster and the Coma cluster. Optical discoveries of clusters continued to accumulate as observing power grew over the next two centuries (see Biviano, 2000, for a review of the history), culminating with the definitive cluster catalogs of George Abell and collaborators Abell, 1958; Abell et al., 1989). Abell's catalogs contain most of the known nearby galaxy clusters and are the foundation for much of our modern understanding of clusters.
Abell recognized that projection effects can complicate the identification of clusters in optical galaxy surveys and therefore was careful in defining his clusters. Working from the Palomar Sky Survey plates he estimated the distance of each cluster candidate from the apparent brightness of its tenth brightest member galaxy. He then counted all the galaxies lying within a fixed projected radius and brighter than a magnitude limit two magnitudes fainter than the third brightest member. The bounding radius, which he determined from the distance estimate, is now known to be $\sim 2 \mathrm{Mpc}$ and was the same for all clusters. $^{2}$ In order to compensate for projection effects, he subtracted from his galaxy counts a background level equivalent to the mean number of galaxies brighter than the magnitude limit for the cluster in similarly-sized, cluster-free regions of the plate, and retained all cluster candidates with a net excess of 50 galaxies brighter than the limiting magnitude.

Most of the optical cluster identification techniques used today extend and refine Abell's basic approach (e.g., Dalton et al., 1997; Lumsden et al., 1992; Postman et al., 1996), often augmenting it with information about galaxy colors (e.g., Bahcall et al., 2003b; Gladders and Yee, 2000; Nichol, 2004). These improvements are necessary because the contrast of clusters against the background galaxy counts decreases with cluster distance. Galaxy colors can help identify distant clusters because many cluster galaxies are significantly redder than other galaxies at a similar redshift, owing to their lack of ongoing star formation. The colors of their aging stellar populations therefore place these cluster members on a narrow and distinctive locus known as the "red sequence" in a plot of galaxy color versus magnitude (e.g. Gladders and Yee, 2000).

Once suitable cluster candidates are found, their status as true mass concentrations can be checked by measuring the underlying mass. Optical observations offer two complementary ways to perform such measurements, through the orbital velocities of the member galaxies and through the degree to which galaxies lying behind the cluster are lensed by the cluster's gravitational potential. We will discuss both of these methods after a few more words about how galaxy counts relate to the overall optical luminosities of clusters.

\section{Optical Richness}

To the extent that light traces mass in the universe, the total optical luminosity of a cluster is itself an indicator of a cluster's mass. Measuring the luminosity of every galaxy in a cluster is impractical, especially for distant clusters in which only the brightest galaxies can be

\footnotetext{
2 The Megaparsec is astronomers' favored unit of distance on cluster scales: $1 \mathrm{Mpc}=3.09 \times 10^{24} \mathrm{~cm}=3.26 \times 10^{6}$ light years.
} 
observed. However, because the luminosity distribution function of cluster galaxies is nearly the same from cluster to cluster, observing the high-luminosity tip of that distribution allows one to normalize the overall galaxy luminosity function for the cluster, yielding estimates for both the cluster's total optical luminosity and its mass.

Abell's catalogs encode this information by placing clusters in categories of "richness" corresponding to the net excess of galaxies brighter than the magnitude limit used to define each cluster. The richest clusters (class 5) contain over 300 galaxies brighter than the magnitude limit, while the poorest (class 1 ) contain only 50-79 such galaxies. Clusters not quite making Abell's cut (3049 galaxies above the magnitude limit) were assigned to richness class zero. Within this system, the Coma cluster originally ranked as richness class 2 .

Invoking assumptions about the shape of the luminosity distribution function helps to link richness more directly to a cluster's total luminosity. Cluster galaxies generally adhere to a luminosity distribution function following the form proposed by Schechter (1976), with the number of galaxies in luminosity range $d L$ about $L$ proportional to $L^{-\alpha} \exp \left(-L / L_{*}\right)$, with $\alpha \approx 1$ (e.g., Balogh et al., 2001a). Assuming this distribution function, Postman et al. (1996) define a richness parameter $\Lambda_{\mathrm{cl}}$ equivalent to the number of cluster galaxies brighter than the characteristic luminosity $L_{*}$. They find that $\Lambda_{\mathrm{cl}}$ is highly correlated with Abell's richness measure, but the scatter between richness and $\Lambda_{\mathrm{cl}}$ is large.

Another richness parameter in current use is $B_{\mathrm{cg}}$, the amplitude of the correlation function between the cluster center and the member galaxies (Longair and Seldner, 1979; Yee and López-Cruz, 1999). It is derived from the angular correlation function of galaxies measured down to a given magnitude limit, after removing the background counts, and is normalized by dividing out the expected luminosity distribution function of galaxies integrated down to that magnitude limit. This richness parameter also correlates with Abell's richness, but again the scatter is broad. Yee and Ellingson (2003) show that $B_{\text {cg }}$ correlates well with other global properties of clusters, suggesting that richness observations may become an inexpensive way to measure cluster masses, but first the mass-richness relation must be calibrated and the scatter in that relation must be quantified.

\section{Galaxy Velocities}

Once a cluster has been optically identified, obtaining the radial velocities $v_{r}$ of the cluster galaxies from their redshifts helps in mitigating projection effects and in measuring the cluster's mass. Because the velocity distribution of a relaxed cluster's galaxies is expected to be gaussian in velocity space, galaxies with velocities falling well outside the best-fitting gaussian envelope are unlikely to be cluster members and are generally discarded. Fitting the velocity distribution $\exp \left[-\left(v_{r}-\left\langle v_{r}\right\rangle\right)^{2} / 2 \sigma_{1 \mathrm{D}}^{2}\right]$ to the remaining galaxies then yields a one-dimensional velocity dispersion $\sigma_{1 \mathrm{D}}$ for the cluster. If the velocity distribution of a cluster candidate is far from gaussian, then it is probably not a real cluster but rather a chance superposition of smaller structures. Obviously, the accuracy of $\sigma_{1 \mathrm{D}}$ depends critically on the number of galaxies with measured velocities and the method for identifying and eliminating non-members.

Zwickv (1933, 1937) was the first to measure a cluster's velocity dispersion, finding $\sigma_{1 \mathrm{D}} \sim 700 \mathrm{~km} \mathrm{~s}^{-1}$ for the Coma cluster. He correctly concluded from this fact and his estimate of the Coma cluster's overall radius that this cluster's mass must be far greater than the observed mass in stars - the first evidence for dark matter in the universe. Shortly thereafter, Smith (1936) showed that the same was true of the Virgo cluster. Zwicky's reasoning involved the virial theorem of classical mechanics, which applies to steady, gravitationally bound systems. Differentiating the system's moment of inertia $I=\sum_{i} m_{i} \mathbf{r}_{i}^{2}$ twice with respect to time and setting the result to zero produces the virial relation

$$
\sum_{i} m_{i} \dot{\mathbf{r}}_{i}^{2}=-\sum_{i} m_{i} \ddot{\mathbf{r}}_{i} \cdot \mathbf{r}_{i}
$$

The left-hand side is twice the total kinetic energy of the cluster's particles, and in a spherically symmetric system of mass $M$ with a gaussian velocity distribution, that kinetic energy is $3 M \sigma_{1 \mathrm{D}}^{2} / 2$. If the system is isolated, then the right-hand side is equal to the absolute value of the gravitational potential energy, which can be expressed as $G M^{2} / r_{G}$, where

$$
\begin{aligned}
r_{G} & \equiv M^{2}\left(\sum_{i} \sum_{i<j} \frac{m_{i} m_{j}}{r_{i j}}\right)^{-1} \\
& \approx \frac{\pi}{2} M^{2}\left(\sum_{i} \sum_{i<j} \frac{m_{i} m_{j}}{r_{\perp, i j}}\right)^{-1}
\end{aligned}
$$

and $r_{i j}$ is the separation between particles $i$ and $j$. The approximation gives $r_{G}$ for a spherically symmetric system in terms of the projected particle separations $r_{\perp . i j}$ in the plane of the sky (Limber and Mathews, 1960). According to the virial theorem, the mass of a spherical, isolated cluster should therefore be $M=3 \sigma_{1 \mathrm{D}}^{2} r_{G} / G$.

Applying this virial analysis to real clusters is not quite so simple because clusters are not isolated systemsthere is no clean boundary separating a cluster from the rest of the universe. Segregating the cluster from the outlying regions with an arbitrary bounding surface alters the interpretation of the right-hand side of equation (11). In a steady state, the momentum flux of particles exiting the boundary is equal to that entering, so the bounding surface is formally equivalent to a reflecting wall that adds a pressure correction term, offsetting some of the gravitational potential energy Carlberg et al., 1997a: The and White, 1986). One must also account for 
objects seen in projection, such as infalling galaxies that have not yet entered into virial equilibrium and interlopers that are not true cluster members, problems that have led to the invention of various kinds of projected mass estimators (Bahcall and Tremaine, 1981; Heisler et al., 1985).

Extensive redshift measurements now allow observers to measure much more than just a cluster's velocity dispersion, enabling detailed studies of a cluster's mass profile and dynamical state. Generally a cluster's velocity dispersion declines with projected radius, implying that the relationship between projected radius and the mass enclosed within that radius is somewhat shallower than linear in the cluster's outskirts (Carlberg et al., 1997a; Kent and Gunn, 1982; Rood et al., 1972). Beyond the approximate virial radius of a cluster, the enclosed mass continues to increase and the galaxies move primarily on infalling radial trajectories (Biviano and Girardi, 2003; Diaferio and Geller, 1997; Kaiser, 1987; Regos and Geller, 1989). Even farther out is a thin region where galaxies are nearly stationary with respect to the cluster because there the cluster's gravity has just succeeded in reducing the outward Hubble flow to a standstill (Kaiser, 1987; Rines et al., 2003). Eventually these galaxies will fall back toward the cluster and become cluster members.

Because clusters are dynamical systems that have not quite finished forming and equilibrating, the velocity dispersion and virial theorem by themselves do not yield an exact cluster mass measurement. Detailed information on the spatial distribution of galaxy velocities is of great help in measuring the masses of large, nearby clusters but similar information is very difficult to obtain for the distant clusters so interesting to cosmologists. In lieu of detailed observations, one can use simulations of cluster formation to calibrate the approximate virial relationship between velocity dispersion and cluster mass, but we will postpone discussion of that procedure to the discussion of dark-matter dynamics in Sec. III]

\section{Gravitational Lensing}

In his remarkable 1937 paper on the Coma cluster, Zwicky also proposed that cluster masses could be measured through gravitational lensing of background galaxies. That technique did not become practical for six more decades but is now one of the primary methods for measuring cluster mass. Lensing is sensitive to the cluster's mass within a given projected radius $r_{\perp}$ because the mass within this radius deflects photons toward our line of sight through the cluster's center. When the deflection angle is small compared to a background galaxy's angular distance from the cluster center, weak lensing shifts each point in the galaxy's image to a slightly larger angular distance from the cluster's center, thereby distorting the image by stretching it tangentially to $r_{\perp}$. Measuring the weak-lensing distortion of any single galaxy is nearly im- possible because the exact shape of the unlensed galaxy is generally unknown. Instead, observers must measure the shear distortion of an entire field of background galaxies, under the assumption that any intrinsic deviations of galaxy images from circular symmetry are uncorrelated.

Many excellent articles explain this weak-lensing technique in more detail (Bartelmann and Schneider, 2001; Hoekstra et al., 1998; Kaiser and Squires, 1993; Mellier, 1999; (Tvson et al., 1990). Here we wish only to give a flavor of how a cluster's mass can be measured from the lensing it induces. The deflection angle itself depends on the gradient of the gravitational potential in the lensing system, meaning that a mass sheet of constant surface density produces no shear and goes undetected. Additional mass that is distributed symmetrically about the line of sight through a cluster's center bends photon paths by an angle twice that expected from Newtonian physics, $4 G M\left(<r_{\perp}\right) / c^{2} r_{\perp}$, which can be measured from the shear distortion and redshift distribution of the background galaxies. Obtaining a cluster mass from the mass $M\left(<r_{\perp}\right)$ along the column bounded by $r_{\perp}$ requires additional assumptions about how mass is distributed within this column. A particularly simple mass configuration would be a singular isothermal sphere, in which $\sigma_{1 \mathrm{D}}$ remains constant with radius and $M(r)=2 \sigma_{1 \mathrm{D}}^{2} r / G$ (Sec. III.B.2); notice that the boundary pressure term required in this configuration alters the usual virial relation. The deflection angle for this mass distribution is $4 \pi \sigma_{1 \mathrm{D}}^{2} / c^{2}$, independent of radius. In general, however, the cluster potential will not be precisely isothermal, nor will the cluster be perfectly spherical.

Simulations of large-scale structure formation suggest that superpositions of other mass concentrations limit the accuracy of weak-lensing masses, at least for clusters defined to be within spherical volumes. Projected mass fluctuations along the line of sight to a distant cluster can be on the order of $\sim 10^{14} M_{\odot}$ Hoekstra, 2001; Metzler et al., 2001, 1999). On the other hand, weaklensing masses are expected to correlate quite well with cluster richness, another measure of the mass within a cylindrical region, raising the possibility that at least some of the projected mass can be accounted for by using galaxy colors to separate these mass concentrations from the cluster in redshift space.

\section{B. Clusters in X-rays}

Clusters of galaxies are X-ray sources because galaxy formation is inefficient. Only about a tenth of the universe's baryons reside with stars in galaxies, leaving the vast majority adrift in intergalactic space. Most of these intergalactic baryons are extremely difficult to observe, but the deep potential wells of galaxy clusters compress the associated baryonic gas and heat it to X-ray emitting temperatures. The gas temperature inferred from a cluster's X-ray spectrum therefore indicates the depth of a cluster's potential well, and the emission-line strengths 
in that spectrum indicate the abundances of elements like iron, oxygen, and silicon in the intracluster medium (ICM). Here we outline the primary characteristics of that X-ray emission. For a more detailed discussion of the physics, see Sarazin (1988).

\section{X-ray Surface Brightness}

Extended X-ray emission from clusters of galaxies was first observed in the early 1970's (Forman et al., 1972; Gurskv et al., 1971; Kellogg et al., 1972), but was correctly attributed to thermal bremsstrahlung several years earlier by Felten et al. (1966), who were inspired by a spurious X-ray detection of the Coma cluster. For typical cluster temperatures $(k T \gtrsim 2 \mathrm{keV})$ the emissivity of thermal bremsstrahlung dominates that from emission lines, but below $\sim 2 \mathrm{keV}$ that situation reverses, given the typical heavy-element abundances relative to hydrogen which are $\sim 0.3$ times those found in the Sun. The rate at which the ICM radiates energy can be expressed in terms of a cooling function $\Lambda_{c}(T)$ computed assuming that collisional ionization equilibrium determines the relative abundance of each ion. Many collisional ionization codes have been developed to compute the emissivity and X-ray spectrum of such gas (e.g., Ravmond and Smith, 1977). Because these cooling processes all involve electrons colliding with ions, the resulting cooling function is usually defined so that either $n_{e} n_{\mathrm{H}} \Lambda_{c}(T)$ or $n_{e} n_{\text {ion }} \Lambda_{c}(T)$ is the luminosity per unit volume. Tozzi and Norman (2001) give a useful fit to the computations of Sutherland and Dopita (1993) for abundances equal to 0.3 times their solar values. For typical ICM temperatures, $\Lambda_{c} \sim 10^{-23} \mathrm{erg} \mathrm{cm}^{3} \mathrm{~s}^{-1}$.

In most clusters, the intracluster gas appears to be in approximate hydrostatic equilibrium. Assuming spherical symmetry, the equation of hydrostatic equilibrium can be written

$$
\frac{d \ln \rho_{g}}{d \ln r}+\frac{d \ln T}{d \ln r}=-2 \frac{T_{\phi}(r)}{T},
$$

where $\rho_{g}$ is the gas density and $k_{\mathrm{B}} T_{\phi}(r)=$ $G M(r) \mu m_{p} / 2 r$ is the characteristic temperature of a singular isothermal sphere with the same value of $M(r) / r$. Making the additional assumption that the gas is isothermal leads to a classic model for the $\mathrm{X}$-ray surface brightness of clusters known as the beta model (Cavaliere and Fusco-Femiand, 1976). If the velocity distribution of the particles responsible for $M(r)$ is also isothermal with a constant velocity dispersion $\sigma_{1 \mathrm{D}}$, then Poisson's equation implies

$$
\frac{d \ln \rho_{g}}{d r}=-\frac{\mu m_{p}}{k T} \frac{d \phi}{d r}=\beta \frac{d \ln \rho}{d r},
$$

where the eponymous $\beta \equiv \mu m_{p} \sigma_{1 \mathrm{D}}^{2} / k T$ (e.g., Sarazin, 1988). Given the approximate isothermal potential of King (1962), $\rho(r) \propto\left[1+\left(r / r_{c}\right)^{2}\right]^{-3 / 2}$, in which $r_{c}$ is a core radius that keeps the profile from becoming singular at the origin, the gas density profile becomes $\rho_{g}(r) \propto\left[1+\left(r / r_{c}\right)^{2}\right]^{-3 \beta / 2}$. The expected X-ray surface brightness profile for an isothermal gas is then $\propto\left[1+\left(r / r_{c}\right)^{2}\right]^{-3 \beta+1 / 2}$, and fitting this model to the observations gives the best-fit parameters $r_{c}, \beta_{\text {fit }}$, and the normalization of the gas-density distribution.

Beta models generally describe the observed surfacebrightness profiles of clusters quite well in the radial range from $\sim r_{c}$ to $\sim 3 r_{c}$, with $\beta_{\text {fit }} \approx 2 / 3$ and $r_{c} \sim 0.1 r_{G}$ giving the best fits for rich clusters (Jones and Forman, 1984) and a possible trend toward lower $\beta$ values in poorer clusters (Finoguenov et al. 2001b; Helsdon and Ponman, 2000; Horner et al., 1999; Sanderson et al., 2003). The X-ray luminosity integrated over radius converges for $\beta>0.5$, meaning that most of the observed X-rays come from a relatively small proportion of the ICM. However, beta models often underestimate the central surface brightness (Jones and Forman, 1984) and tend to overestimate the brightness at $r \gg r_{c}$ (Vikhlinin et al., 1999). These discrepancies arise in part because the intracluster medium is not strictly isothermal (Sec. III.C.2) and because real cluster potentials differ from the King model (Sec. III.B.2).

The centrally concentrated surface-brightness profiles of clusters make X-ray surveys very effective at finding cluster candidates. Because X-ray emission depends on density squared, clusters of galaxies strongly stand out against regions of lesser density, minimizing the complications of projection effects (see Rosati et al., 2002, for a recent review). Surveys of X-ray selected clusters currently extend to $z \approx 1.3$ (e.g. Rosati et al., 2004; Stanford et al., 2001), a limit owing to the decline of surface brightness with redshift (Sec. III.A.2). Unfortunately, X-ray luminosity correlates less well than one would like with the optical properties of clusters. Early studies showed that X-ray luminosity correlates with optical richness but with a large scatter (Bahcall, 1977; Mushotzkv, 1984), and that situation has not improved much in the intervening decades (Donahue et al., 2002; Gilbank et al., 2003; Kochanek et al., 2003). The optical properties of very luminous X-ray clusters are well behaved (Lewis et al., 1999), but deep optical surveys have found distant cluster candidates that appear to have velocity dispersions much larger than one would guess from their X-ray luminosity (Lubin et al., 2004). These objects may be may be superpositions of smaller clusters whose joint velocity distribution seems like that of a larger relaxed cluster.

\section{Plasma Temperature}

Clusters in hydrostatic equilibrium have a plasma temperature that is closely related to the overall mass. Measuring that temperature requires higher quality data than a simple luminosity measurement, because the photons must be divided among multiple energy bins. Ideally, 
one would like enough data to measure both $T(r)$ and $\rho_{g}(r)$, in which case equation (3) can be solved directly for $M(r)$. Even with the highest-quality data, the derived mass is still slightly model dependent because $T(r)$ and $\rho(r)$ must be determined by deprojecting the surfacebrightness information (Fabian et al., 1981; Kriss et al., 1983; Pizzolato et al., 2003; White et al., 1997).

In practice, the quality of the mass measurement depends on what the total number of observed X-ray photons allows. With limited information about the temperature gradient, one can fit a polytropic $\operatorname{law}^{3} T \propto \rho_{g}^{\gamma_{\text {eff }}-1}$, giving the radial dependence of temperature in terms of an effective adiabatic index $\gamma_{\text {eff }}$ with density as the radial coordinate. However, data on distant clusters often do not allow a temperature gradient to be measured and sometimes are even insufficient to give an accurate temperature. In those cases, one must rely on scaling laws that connect X-ray luminosity with temperature and temperature with mass, calibrated with either high-quality observations or numerical simulations of cluster formation that include all the relevant physics (Sec. III.C).

Limitations in the measurement of cluster temperature systematically affect the mass one infers for the cluster. If only a single temperature can be measured, then the isothermal beta model implies

$$
\frac{M(r)}{r}=\frac{3 \beta k_{\mathrm{B}} T}{G \mu m_{p}} \frac{\left(r / r_{c}\right)^{2}}{1+\left(r / r_{c}\right)^{2}} .
$$

Note that at large radii this relation approaches the one for isothermal gas in a singular isothermal potential, $M(r) / r=2 k_{\mathrm{B}} T / G \mu m_{p}$, as long as $\beta=2 / 3$. However, single temperatures gleaned from a cluster's overall spectrum need to be treated with caution. Global cluster temperatures quoted in the literature are generally spectral-fit temperatures $\left(T_{\mathrm{sp}}\right)$ obtained by fitting a single-temperature emission model to an overall cluster spectrum containing multiple temperature components. These spectral-fit temperatures are similar to, but not identical to, the cluster's luminosity-weighted temperature $T_{\text {lum }}$ in which each temperature component is weighted by $\rho_{g}^{2}$. Numerical simulations indicate that both $T_{\mathrm{sp}}$ and $T_{\text {lum }}$ can differ from the mass-weighted gas temperature $T_{a}$ and from one another by $\sim 10-20 \%$ (Mathiesen and Evrard, 2001; Mazzotta et al., 2004).

A modest amount of spatially resolved temperature information improves the mass measurement. Allowing for a temperature gradient corresponding to $T \propto \rho_{g}^{\gamma_{\text {eff }}-1}$ changes the estimated mass to

$$
\frac{M(r)}{r}=\frac{3 \beta \gamma_{\mathrm{eff}} k_{\mathrm{B}} T(r)}{G \mu m_{p}} \frac{\left(r / r_{c}\right)^{2}}{1+\left(r / r_{c}\right)^{2}} .
$$

\footnotetext{
${ }^{3}$ Note that this is not an actual equation of state for the gas but only a fitting formula for $T(r)$ as a function of $\rho_{g}(r)$.
}

Observers are still working toward a consensus on the temperature gradients of clusters (De Grandi and Molendi, 2002: Irwin and Breoman, 2000; Markevitch et al., 1998; Mushotzkv, 2004; Pratt and Arnaud, 2002), but measured values of $\gamma_{\mathrm{eff}}$ often range as high as 1.2 (Finoguenov et al., 2001b). Cluster temperatures are extremely difficult to observe in the neighborhood of the virial radius, but extrapolating a $\gamma_{\text {eff }}=1.2$ gradient to $10 r_{c}$ leads to a gas temperature less than half the core temperature. Including temperature-gradient information can therefore lower the estimated mass for a cluster of temperature $T_{\text {lum }}$ by up to $\sim 50 \%$.

Despite the potential for systematic uncertainties, the luminosity-weighted temperatures of clusters correlate well with their velocity dispersions. Most of the recent comparisons for low-redshift clusters find that $\sigma_{1 \mathrm{D}} \propto T_{\mathrm{sp}}^{\sim 0.6}$, slightly steeper than expected if both quantities track cluster mass (Lubin and Bahcall, 1993; Xue and Wu, 2000). Those same comparisons find normalizations of this relation for rich clusters in the range $\beta_{\mathrm{sp}}=\mu m_{p} \sigma_{1 \mathrm{D}}^{2} / k T_{\mathrm{sp}}=0.9-1.0$ (Figure 1). The discrepancy between $\beta_{\mathrm{sp}}$ and $\beta_{\mathrm{fit}}$ is no cause for concern. It arises because the true mass profile is not a King model and because clusters are not in perfect hydrostatic equilibrium (Bahcall and Lubin, 1994; Evrard, 1990). More worrisome are recent observations suggesting that the $\mathrm{X}$-ray temperatures of distant optically-selected clusters with unusually small X-ray luminosities are also considerably cooler than their velocity dispersions would indicate (Lubin et al., 2004). However, more extensive redshift measurements have shown that at least one of these systems is composed of several smaller systems that have not yet merged to form a single large cluster Gal and Lubin, 2004).

\section{Measuring Abundances}

Abundances of elements like iron, oxygen, and silicon in the intracluster medium are relatively easy to measure from their emission line fluxes, as long as the temperature of the line-emitting gas is well defined. Because of the low density of intracluster gas, collisional deexcitation is negligible, so every collisional excitation produces a photon that leaves the cluster. Thus, one can fit the optically-thin spectrum of a collisionally-ionized, singletemperature plasma to the observed spectrum, adjusting the abundances in the model to produce the best fit. The high spectral resolution of today's X-ray observatories, Chandra and XMM-Newton, allows abundance determinations for individual elements if enough photons can be gathered. Otherwise, the solar pattern of abundance ratios is assumed for elements other than $\mathrm{H}$ and $\mathrm{He}$ and the normalization of the overall pattern is fit to the observations. Because the most abundant elements are almost completely ionized in the hottest clusters, these abundance determinations depend heavily on the strength of 


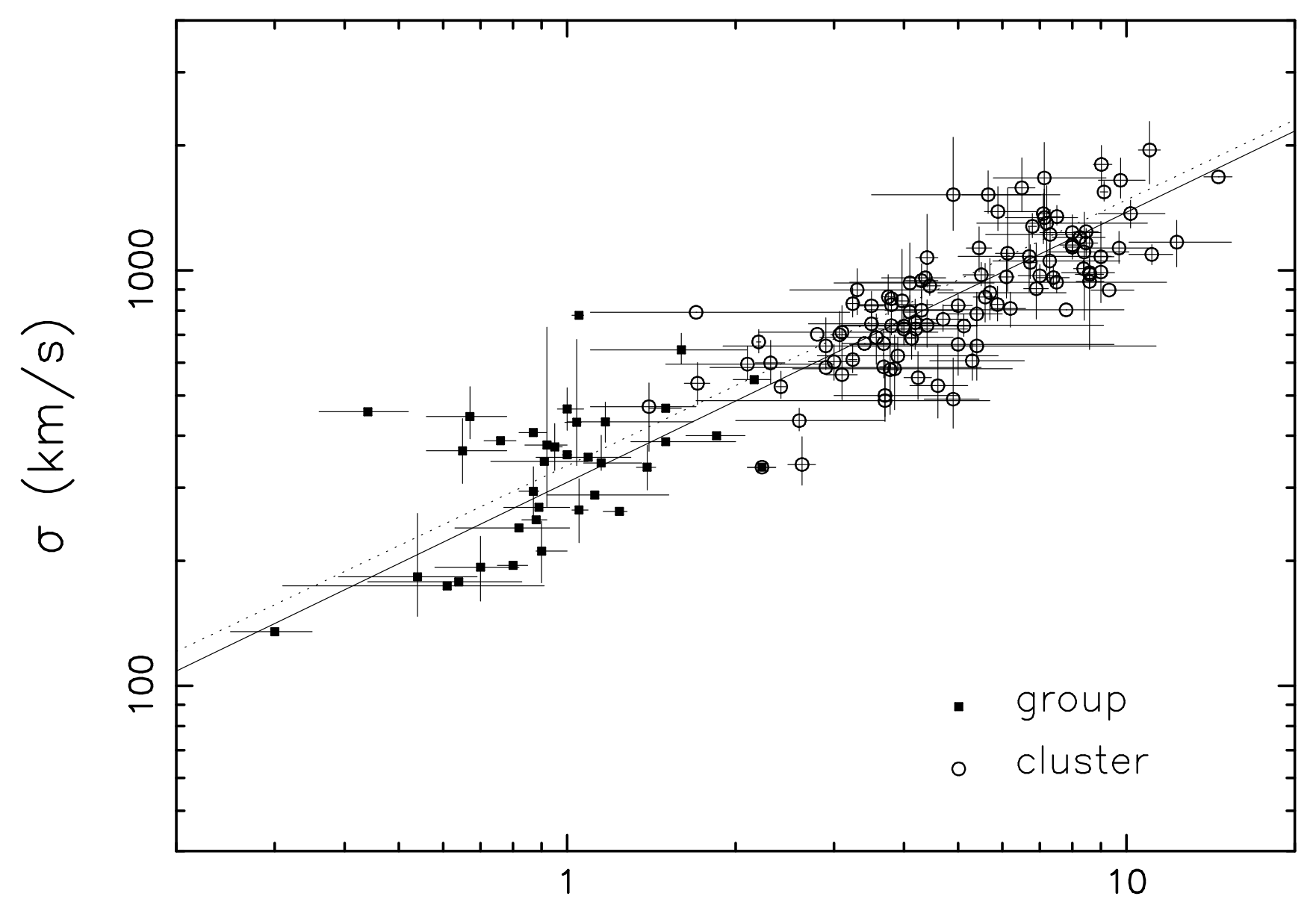

$\mathrm{T}(\mathrm{keV})$

FIG. 1 Relation between velocity dispersion and temperature for a heterogeneous sample drawn from the literature. Solid squares illustrate data on galaxy groups and open circles give the cluster data. The dotted and solid lines show the best power-law fits for groups and clusters, respectively. The best-fitting relation to the combined sample is $\sigma_{1 \mathrm{D}}=$ $10^{2.51 \pm 0.01} \mathrm{~km} \mathrm{~s}^{-1}(T / 1 \mathrm{keV})^{0.61 \pm 0.01}$, corresponding to $\beta_{\mathrm{sp}}=0.97$ at $6 \mathrm{keV}$. (Figure from Xue and Wu, 2000)

the K-shell emission lines of iron, sometimes the only lines that are measurable.

On average, the overall abundances of heavy elements with respect to hydrogen in clusters are about 0.3 times the solar ratios. Just as with temperature, this determination is weighted toward the cluster core because of the $\rho_{g}^{2}$ emissivity. Spatially resolved observations of Fe $K$ line emission show that iron abundances, at least, can be higher at the cluster's center, particularly when a giant, central-dominant galaxy is there. This iron excess is consistent with being supernova debris from the giant galaxy's stars (De Grandi et al., 2003). Farther out in clusters, these Fe gradients appear to flatten at $\sim 0.3$ times the solar level, extending to about $\sim 5 r_{c}$, beyond which point the X-ray surface brightness is too low for accurate abundance and temperature measurements. This abundance level does not seem to have sub- stantially changed from redshift $z \sim 1$ to the present (Donahue et al., 1998, 1999; Tozzi et al., 2003).

The total amount of iron implied by extrapolating this ratio over an entire cluster is quite impressive, exceeding the total amount of iron contained within all the stars in the cluster's galaxies (Renzini, 1997). Explaining how all that iron got into the intracluster medium is challenging. It is comparable to the total amount of iron produced by all the supernovae thought to have exploded during the history of the cluster, and according to some estimates, it requires a disproportionately large number of massive stars to have formed in order to produce enough supernovae (David et al., 1991; Gibson and Matteucci, 1997; Loewenstein, 2001; Loewenstein and Mushotzkv, 1996; Matteucci and Gibson, 1995; Portinari et al., 2003).

Presumably all these supernovae could have driven strong gaseous outflows known as galactic winds that ex- 
pelled the heavy elements into the intracluster medium (Heckman et al., 1990; Larson and Dinerstein, 1975). However, such powerful galactic winds are hard to produce in numerical simulations of galaxies because much of the energy released by massive-star (Type II) supernovae is transferred to cool gas within the galaxy, where it is radiated away before it manages to drive a powerful wind (Mac Low and Ferrara, 1999). Alternatively, some of this iron may come from exploding white dwarfs (Type Ia supernovae), whose iron yields are higher than those of Type II supernovae. In either case, the total amount of kinetic energy released by the supernovae that created these elements is enormous, corresponding to $\sim 0.3-1 \mathrm{keV}$ per particle in the intracluster medium (Finoguenov et al., 2001a; Pipino et al., 2002). Yet, the efficiency of energy transfer from supernovae to the ICM remains an open question (Kravtsov and Yepes, 2000).

In principle, one can probe the origins of elements in the ICM and assess whether massive stars were disproportionately common earlier in time by comparing the abundances of massive-star products like oxygen to that of iron, which may come largely from Type Ia supernovae. No clear answer has yet emerged from such studies, which depend heavily on a proper understanding of the gas temperature distribution to get the correct elemental abundances (e.g., Buote et al., 2003). Some studies have concluded that the relative abundance patterns in the intracluster medium are near solar, implying that the stellar populations producing those supernovae were similar to those in our own galaxy (Renzini, 2004). Other studies find an excess of oxygen and other elements of similar atomic number, suggesting that the cluster's galaxies produced an unusually large number of massive stars early in the cluster's history (e.g., Finoguenov et al., 2003).

\section{Clusters in Microwaves}

Hot gas in clusters can also be observed through its effects on the cosmic microwave background. The background itself has a virtually perfect blackbody spectrum (Mather et al., 1990). Soon after the discovery of this background radiation, Wevmann (1965, 1966) computed how Compton scattering would distort its spectrum, slightly shifting some of the microwave photons to higher energies as they passed through hot intergalactic gas. Sunvaev and Zeldovich $(1970,1972)$ then predicted that hot gas in clusters of galaxies would indeed produce such a distortion, now known as the Sunyaev-Zeldovich (S-Z) effect.

\section{The S-Z Effect}

Two decades after this prediction there were only a few marginal detections (Birkinshaw, 1991), but many clusters were detected at high significance in the ensuing decade (Birkinshaw, 1999; Carlstrom et al., 2000). With multiple new and highly capable S-Z instruments coming on line in the next few years, another quantum leap in this area is poised to happen, enabling wide-field cosmological studies of clusters to extend through much of the observable universe (Carlstrom et al., 2002). A number of recent reviews elucidate the details of the S-Z effect (e.g., Birkinshaw, 1999; Carlstrom et al., 2002). Here we summarize only a few fundamentals.

To lowest order, the shape of the distorted spectrum depends on a single parameter proportional to the product of the probability that a photon passing through the cluster will Compton scatter and the typical amount of energy a scattered photon gains:

$$
y=\int \frac{k_{\mathrm{B}} T}{m_{e} c^{2}} n_{e} \sigma_{\mathrm{T}} d l,
$$

where $\sigma_{\mathrm{T}}$ is the Thomson cross-section and the integral is over a line of sight through the cluster. Because the optical depth of the cluster is small, the change in microwave intensity at any frequency is linearly proportional to $y \ll 1$, with reduced intensity at long wavelengths and enhanced intensity at short wavelengths. Relativistic corrections in hot clusters add a slight frequency dependence to the magnitude of the effect, making cluster temperatures measurable with precise observations of the microwave distortion at several frequencies (see Carlstrom et al., 2002, for a discussion). A cluster's motion with respect to the microwave background produces additional distortion, known as the kinetic S-Z effect, but here we will concern ourselves only with the thermal S-Z effect.

Cosmological applications of the thermal S-Z effect in clusters benefit greatly from the fact that the effect is independent of distance, unlike optical and X-ray surface brightness. Thus, a dedicated S-Z cluster survey efficiently finds clusters out to arbitrarily high redshifts. Because not all these clusters will be well resolved, the surveys will be measuring an integrated version of the distortion parameter:

$$
Y=\int y d A \propto \int n_{e} T d V
$$

where the first integral is over a cluster's projected surface area and the second is over its volume. The $Y$ parameter therefore tells us the total thermal energy of the electrons, from which one easily derives the total gas mass times its mass-weighted temperature within a given region of space. If these regions can be chosen so that the gas mass is always proportional to the cluster's total mass, then the observable $Y$ can be used a measure of cluster mass, once the relationship between $Y$ and mass has been calibrated.

The impressive power of the S-Z effect for finding distant clusters also has a significant drawback, namely sky confusion owing to projection effects. Along any line of sight through the entire observable universe, the probability of passing within the virial radius of a cluster or 
group of galaxies is of order unity (e.g., Voit et al., 2001). Because a cluster's S-Z distortion does not diminish with distance, many of the objects in a highly sensitive S-Z survey will therefore significantly overlap. Information on galaxy colors will help to separate nearby objects from more distant ones, but the implications of sky confusion for making accurate mass measurements are still a matter to be reckoned with (e.g., White et al., 2002). One way to avoid the problem of sky confusion will be to measure the statistical S-Z properties of clusters in the angular power spectrum of the microwave sky instead of analyzing the clusters themselves (da Silva et al., 2001; Holder and Carlstrom, 2001; Seliak et al., 2001). In fact, this statistical signal may already have been detected (Kuo et al., 2004; Pearson et al., 2003)

\section{Comparing $\mathrm{S}-\mathrm{Z}$ with $\mathrm{X}$-ray}

Comparisons between a cluster's X-ray properties and $\mathrm{S}-\mathrm{Z}$ properties are useful in several different ways. Xray observations are nicely complementary to $\mathrm{S}-\mathrm{Z}$ observations of clusters because they give the integral of $\rho_{g}^{2}$ along lines of sight through a cluster in addition to a gas temperature. Assuming that clusters are spherical objects with smooth gas distributions, one can divide the product of temperature and the line-of-sight integral of $\rho_{g}^{2}$ by the observed $y$ value to obtain a cluster's gas density profile. Combining the data in this way can be particularly useful in studying the outskirts of clusters, where the X-ray surface brightness is difficult to observe but the S-Z signal remains substantial. With this density profile in hand, one can then derive the line-of-sight thickness of the cluster from either the X-ray or S-Z observations. This type of information could help to solve the $\mathrm{S}-\mathrm{Z}$ projection problem in fields where there are highquality X-ray and S-Z data.

If a cluster is indeed spherical, then a comparison of its cluster's physical thickness with its apparent angular size directly gives the cluster's distance, which can be used to determine the scale and geometry of the universe (Birkinshaw et al., 1991). Deriving the scale of the universe in this way is subject to numerous systematic effects. For example, clusters are not all perfectly spherical. Many appear slightly ellipsoidal in X-ray images, calling for a sample of clusters with random orientations to beat down this systematic effect, although three-dimensional reconstructions are possible with the addition of gravitational-lensing data (e.g. Zaroubi et al., 2001). Note also that comparisons of X-ray images to S-Z images would produce nonsensical distances if the intracluster medium were highly clumpy, owing to the $\rho_{g}^{2}$ $\mathrm{X}$-ray emissivity. The fact that cluster distances found in this way are consistent with the standard calibrations of Hubble's Law indicates that the X-ray emitting gas is well-behaved and that most clusters are in approximate hydrostatic equilibrium.

\section{EVOLUTION OF THE DARK COMPONENT}

Cluster masses measured with the techniques outlined in the previous section range from around $10^{14} M_{\odot}$ to more than $10^{15} M_{\odot}$, the vast majority of which appears to be dark matter that emits no detectable radiation. Even using alternative theories of gravity, it is difficult to explain the cluster observations without dark matter dominating the overall mass (Sanders, 2003). In contrast, explaining the characteristics of clusters and their evolution with redshift is much easier with models in which non-baryonic cold dark matter dominates the mass density of the universe.

This section explains how the evolution of the dark component of the universe, including both dark matter and dark energy, is thought to be reflected in the evolution of cluster properties. It begins with a summary of the concordance model for cosmology and some closely related alternatives, all of which are predicated on the existence of non-baryonic cold dark matter. It then explains how dark matter drives cluster formation in such models, providing some simple analytical approximations to the extensive numerical work that has been done on the subject. These models do a good job of accounting for the basic properties of observed clusters, allowing astronomers to measure several of the parameters in the concordance model using cluster observations, most notably the overall mass density of the universe and the amplitude of the initial spectrum of density perturbations that eventually produces all the structure we observe.

The accuracy of those parameter measurements is currently limited by uncertainties in the relationships between cluster masses and the observable properties that trace those masses. Numerical simulations of cluster formation do not yet provide precise calibrations of these relations because they do not yet account for all of the thermodynamical processes associated with galaxy formation. The third part of this section surveys the mass-observable relations and how the uncertainties in those relations affect cosmological parameters derived from them. The fourth part of this section examines how the properties of clusters evolve and how fitting that evolution with cosmological models improves the accuracy of the derived cosmological parameters. Even though current surveys of distant clusters contain relatively few objects, they already place strong constraints on the overall matter density. Larger cluster surveys in both the microwave and X-ray bands have the potential to place much stronger constraints on the overall cosmological model, measuring both dark matter and dark energy parameters to $5 \%$ statistical accuracy, independently of other cosmological observations.

\section{A. A Recipe for the Universe}

Our current understanding of cluster evolution is an outgrowth of the overall cosmological model, whose pri- 
mary features depend on just a handful of parameters. One set of parameters specifies the global cosmological model, which describes the overall geometry of the universe, the mean density of its contents, and how its scale changes with time. The other important set of parameters specifies the initial spectrum of density perturbations that grew into the galaxies and clusters of galaxies we see today. Here we define both sets of parameters and their roles in the context of the overall model. More extensive and detailed discussions of this recipe for the universe can be found in some of the excellent books on cosmology (e.g., Peacock, 1999; Peebles, 1993).

\section{Global Dynamics}

The expansion of the universe can be characterized by a time-dependent scale factor $a(t)$ proportional to the mean distance between the universe's galaxies. Hubble's Law relating the distance $d$ between two galaxies and the speed $v$ at which they appear to move apart can then be written as $v=H(t) d$, where $H(t)=\dot{a} / a$ is the Hubble parameter. Many independent measurements indicate that the value of this parameter at the current time $t_{0}$ is $H\left(t_{0}\right)=H_{0}=71 \pm 7 \mathrm{~km} \mathrm{~s}^{-1} \mathrm{Mpc}^{-1}$ (Freedman et al., 2001). The value of $H_{0}$, known as Hubble's constant, is often further distilled in the literature into the dimensionless quantity $h=H_{0} /\left(100 \mathrm{~km} \mathrm{~s}^{-1} \mathrm{Mpc}^{-1}\right)$. Sometimes this review will use the more suitable alternative $h_{70}=H_{0} /\left(70 \mathrm{~km} \mathrm{~s}^{-1} \mathrm{Mpc}^{-1}\right)$ when characterizing observable cluster properties.

On very large scales, the universe appears homogenous and isotropic. Astronomers therefore assume that the time-dependent behavior of $H(t)$ obeys the FriedmannLemaitre model of the universe, in which

$$
\frac{\ddot{a}}{a}=-\frac{4}{3} \pi G\left(\rho+\frac{3 p}{c^{2}}\right)
$$

where $\rho(t) c^{2}$ is the mean density of mass-energy and $p(t)$ is the pressure owing to that energy density. Local energy conservation requires that

$$
\dot{\rho} c^{2}=-3 \frac{\dot{a}}{a}\left(\rho c^{2}+p\right),
$$

and we can use this expression to integrate the dynamical equation as long as we know the equation of state linking $\rho$ and $p$. If the equation of state has the form $p=w \rho c^{2}$, then density changes with the expansion as $\rho \propto a^{-3(1+w)}$. For a single mass-energy component with a constant value of $w$ we therefore obtain

$$
\dot{a}^{2}=\frac{8 \pi G}{3} \rho_{0} a^{-(1+3 w)}+\text { const. , }
$$

where $\rho_{0}$ is the value of the energy density when $a=1$ and the constant of integration is related to the global curvature of the universe.
It is most convenient to normalize the scale factor so that it equals unity at the current time. Then the cosmological redshift $z$ of radiation from distant objects is simply related to the scale factor of the universe when that radiation was emitted: $a=(1+z)^{-1}$. This definition allows us to link the constant of integration to more familiar parameters, obtaining

$$
\left(\frac{\dot{a}}{a}\right)^{2}=H_{0}^{2}\left[\Omega_{0}(1+z)^{3(1+w)}+\left(1-\Omega_{0}\right)(1+z)^{2}\right],
$$

where $\Omega_{0}$ is the current energy density $\rho_{0}$ in units of the current critical density $\rho_{\text {cr } 0}=3 H_{0}^{2} / 8 \pi G$.

Several different components of the universe, each with a different equation of state, can influence the overall expansion history. Non-relativistic particles with a mass density $\rho_{\mathrm{M}}$ contribute negligible pressure, corresponding to $w=0$. The energy density $\rho_{\mathrm{R}} c^{2}$ in photons and other relativistic particles exerts a pressure with $w=1 / 3$. Einstein's cosmological constant acts like an energy density $\rho_{\Lambda} c^{2}$ that remains constant while the universe expands and therefore exerts a pressure corresponding to $w=-1$. Including each of these components yields the dynamical equation

$$
\begin{array}{r}
H^{2}(z)=\left(\frac{\dot{a}}{a}\right)^{2}=H_{0}^{2}\left[\Omega_{\mathrm{M}}(1+z)^{3}+\Omega_{\mathrm{R}}(1+z)^{4}\right. \\
\left.+\Omega_{\Lambda}+\left(1-\Omega_{0}\right)(1+z)^{2}\right]
\end{array}
$$

where $\Omega_{x}$ is the current mass-energy density in component $x$ in units of $\rho_{\text {cro }}$ and $\Omega_{0}=\Omega_{\mathrm{M}}+\Omega_{\mathrm{R}}+\Omega_{\Lambda}$. The value of $\Omega_{x}$ at an arbitrary redshift is given by $\Omega_{x}(z)=\Omega_{x}(1+z)^{3(1+w)}\left[H(z) / H_{0}\right]^{-2}$.

Each of these energy-density parameters can be further articulated. The matter density parameter $\Omega_{\mathrm{M}}$ consists of a contribution $\Omega_{\mathrm{b}}$ from baryons and a contribution $\Omega_{\mathrm{CDM}}$ from non-baryonic cold dark matter. The radiation density parameter includes contributions from the photons of the microwave background, $\Omega_{\mathrm{CMB}}$, and from relict neutrinos produced in the Big Bang, $\Omega_{\nu}$, as long as they remain relativistic particles. Finally, because the physical origin of the $\Omega_{\Lambda}$ term remains mysterious, it may not be correct to assume that the energy density responsible for it stays constant with time. In order to check this possibility observationally, one can replace the $\Omega_{\Lambda}$ term with a generalized dark-energy term $\Omega_{\Lambda}(1+z)^{3(1+w)}$ and attempt to measure the value of $w$ (Turner and White, 1997; Wang and Steinhardt, 1998).

Recent observations, including the cluster studies we will discuss later, have provided approximate values for many of these energy-density parameters, allowing us to estimate when each of the various energy components dominated the dynamics (Figure 2). Dark energy with $\Omega_{\Lambda} \approx 0.7$ seems to be most important at the current epoch, and because of the scaling of other terms with redshift, it will grow increasingly dominant as time progresses. Non-relativistic matter appears to have a density corresponding to $\Omega_{\mathrm{M}} \approx 0.3$, implying that matter dominated the dynamics at $z \gtrsim 1$. The radiation term was 


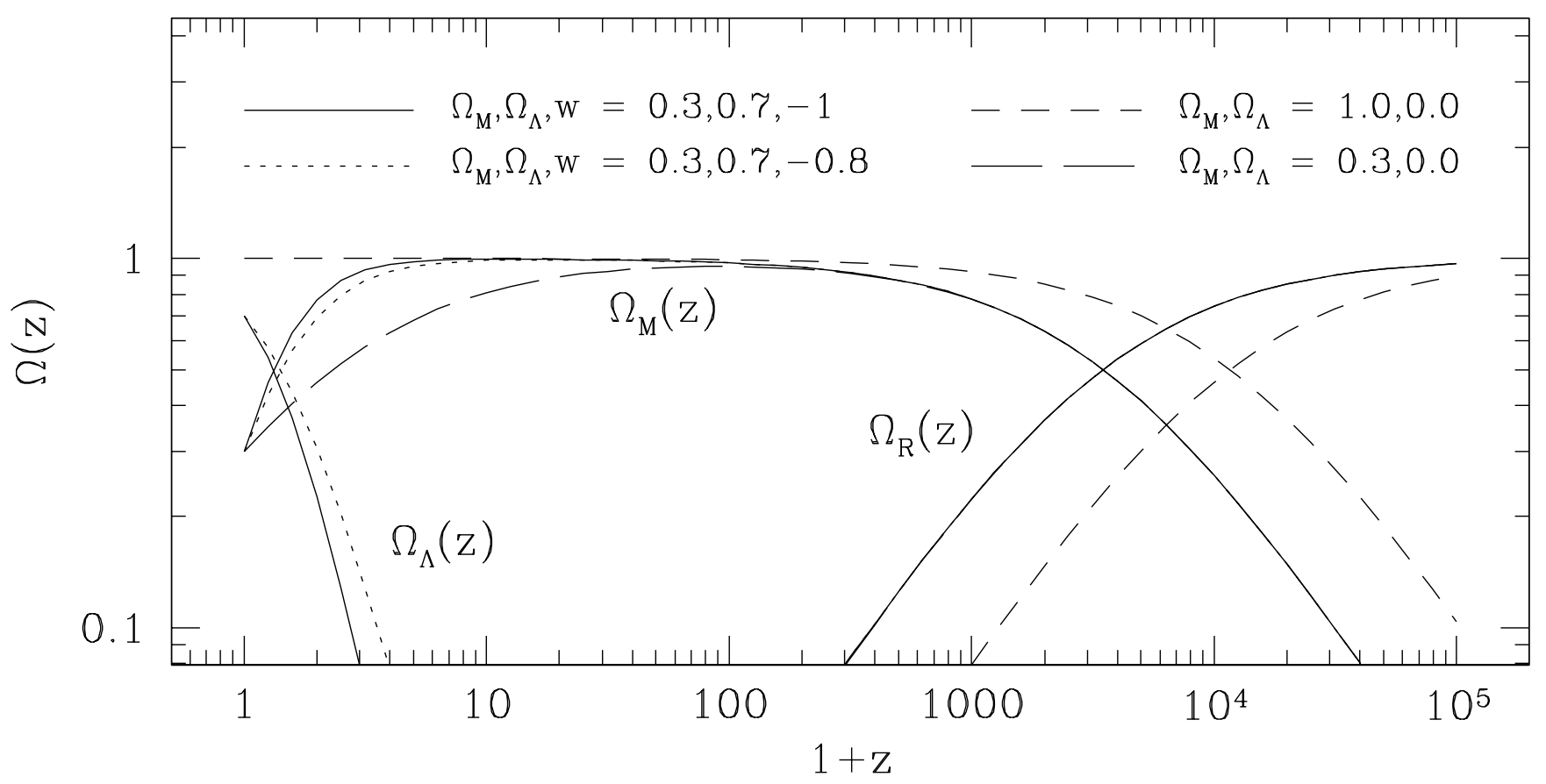

FIG. 2 Evolution of energy densities with redshift. The various lines show the dependence of $\Omega_{\mathrm{M}}(z), \Omega_{\Lambda}(z)$, and $\Omega_{\mathrm{R}}(z)$ on redshift for various sets of present-day cosmological parameters. Structure in the universe grows most rapidly while $\Omega_{\mathrm{M}}(z) \approx 1$, because positive density perturbations then exceed the critical density. This period of time occurs between the redshift $z_{\text {eq }}$ when $\Omega_{\mathrm{M}}\left(z_{\mathrm{eq}}\right)=\Omega_{\mathrm{R}}\left(z_{\mathrm{eq}}\right)$ and the redshift at which $\Omega_{\mathrm{M}}$ begins to drop. Notice that the redshift $z_{\text {eq }}$ is earlier for larger present-day values of $\Omega_{\mathrm{M}}$ and that the redshift at which $\Omega_{\mathrm{M}}(z)$ begins to decline depends on the characteristics of dark energy. Observations of clusters and their evolution provide opportunities to constrain the values of $\Omega_{\mathrm{M}}, \Omega_{\Lambda}$, and $w$ because the timing of both of these epochs influences the properties of the cluster population.

most important in the distant past, prior to the redshift $z_{\mathrm{eq}}=\Omega_{\mathrm{M}} / \Omega_{\mathrm{R}}-1$ of matter-radiation equality. Neutrinos with masses less than a few eV will be relativistic particles at this epoch, leading to

$$
z_{\text {eq }}=2.37 \times 10^{4} \Omega_{\mathrm{M}} h^{2}
$$

for $T_{\mathrm{CMB}}=2.73 \mathrm{~K}$ at $z=0$ and three families of neutrinos.

\section{Global Geometry}

Geometry in a universe that is homogenous and isotropic has the same radius of curvature everywhere, but its overall architecture can be either positively curved, flat, or negatively curved, depending on the value of $\Omega_{0}$. Because the scale of the universe is changing with time, the most sensible coordinate system to use when describing its geometry is one that expands along with the universe. In such a comoving coordinate system, a radial interval in spherical coordinates has length $a(t) d r$, and the interval corresponding to a small transverse angle $d \psi=\sqrt{d \theta^{2}+\sin ^{2} \theta \cdot d \phi^{2}}$ depends on the radius of curvature $a(t) R_{\kappa}$. For positive curvature, analogous to the surface of a sphere, the transverse interval is $a(t) R_{\kappa} \sin \left(r / R_{\kappa}\right) d \psi$, and for negative curvature it is $a(t) R_{\kappa} \sinh \left(r / R_{\kappa}\right) d \psi$.

We can therefore write the Robertson-Walker metric that describes such a universe as

$$
c^{2} d \tau^{2}=c^{2} d t^{2}-a^{2}(t)\left[d r^{2}+R_{\kappa}^{2} S_{\kappa}^{2}\left(r / R_{\kappa}\right) d \psi^{2}\right],
$$

where $S_{\kappa}(x)=\sin x$ for positive curvature $(\kappa=1)$, $S_{\kappa}(x)=\sinh x$ for negative curvature $(\kappa=-1)$, and a flat universe $(\kappa=0)$ corresponds to $R_{\kappa} \rightarrow \infty$. The metric can be written in the more familiar form

$$
c^{2} d \tau^{2}=c^{2} d t^{2}-a^{2}(t)\left[\frac{d r_{\kappa}^{2}}{1-\kappa r_{\kappa}^{2} / R_{\kappa}^{2}}+r_{\kappa}^{2} d \psi^{2}\right]
$$

with the definition $r_{\kappa} \equiv R_{\kappa} S_{\kappa}\left(r / R_{\kappa}\right)$. Plugging this metric into Einstein's field equations leads to

$$
H^{2}(z)=\left(\frac{\dot{a}}{a}\right)^{2}=\frac{8 \pi G \rho}{3}-\frac{\kappa c^{2}}{a^{2} R_{\kappa}^{2}},
$$

which relates the radius of curvature to other cosmological parameters:

$$
R_{\kappa}=\frac{c}{H_{0}} \sqrt{\frac{\kappa}{\Omega_{0}-1}} .
$$

Notice that the universe at early times is effectively flat as long as $\Omega_{\mathrm{M}}+\Omega_{\mathrm{R}}>0$ because the horizon size of 
the observable patch is $\sim c / H(z) \ll(1+z)^{-1} R_{\kappa}$ for observers at times corresponding to large values of the redshift $z$.

The low-redshift universe may also be effectively flat, but that is not guaranteed. Consequently, both the expansion of the universe and its curvature need to be taken into account when we observe highly redshifted objects like distant clusters of galaxies. Because the metric relates the comoving radial coordinate $r$ to redshift through $d r / d z=-c / H(z)$, the coordinate distance to an object with an observed redshift $z$ is

$$
r(z)=c \int_{0}^{z} \frac{d z}{H(z)} .
$$

Relations involving the divergence of light paths can then be compactly written in terms of $r_{\kappa}(z)=R_{\kappa} S_{\kappa}\left[r(z) / R_{\kappa}\right]$, which reduces to $r(z)$ in a flat universe. For example, the angle subtended at coordinate distance $r(z)$ by the transverse length $l$ becomes

$$
\psi=\frac{(1+z) l}{r_{\kappa}(z)} .
$$

In a flat, static universe, an object of physical size $l$ would subtend this same angle if it were at the distance $d_{\mathrm{A}}(z)=$ $r_{\kappa}(z) /(1+z)$, sometimes called the angular-size distance. Likewise, the comoving volume within a solid angle $d \Omega$ and a redshift interval $d z$ is given by

$$
\frac{d^{2} V_{\mathrm{co}}}{d \Omega d z}=\frac{c r_{\kappa}^{2}(z)}{H(z)} .
$$

These formulae are useful to cluster cosmology because they allow us to constrain $H(z)$ and the cosmological parameters that go into it if we know either the transverse sizes of high-redshift clusters or their number density within a given comoving volume. Figure 3 shows how the comoving volume of the universe depends on redshift for several different sets of cosmological parameters.

When surveying the universe for clusters, we also need to know how the geometry and expansion of the universe affect the apparent brightness of a cluster and the galaxies within it. The expansion alone reduces the energy flux received from a distant object by two factors of $1+z$, with one factor coming from the time dilation of the photon flux owing to expansion and the other from the redshift of the photons themselves. The observed energy flux from an object of luminosity $L$ is therefore

$$
F=\frac{L}{4 \pi(1+z)^{2} r_{\kappa}^{2}(z)} .
$$

We would measure the same flux from an equivalent object in a flat, static universe if it were at a distance $d_{L}(z)=(1+z) r_{\kappa}(z)$, sometimes called the luminosity distance. The consequences for surface brightness, equal to flux per unit solid angle, are even more dramatic. In a flat, static universe, an object's surface brightness remains constant, but its surface brightness in an expanding universe is reduced by a factor $d_{A}^{2} / d_{L}^{2} \propto(1+z)^{-4}$,

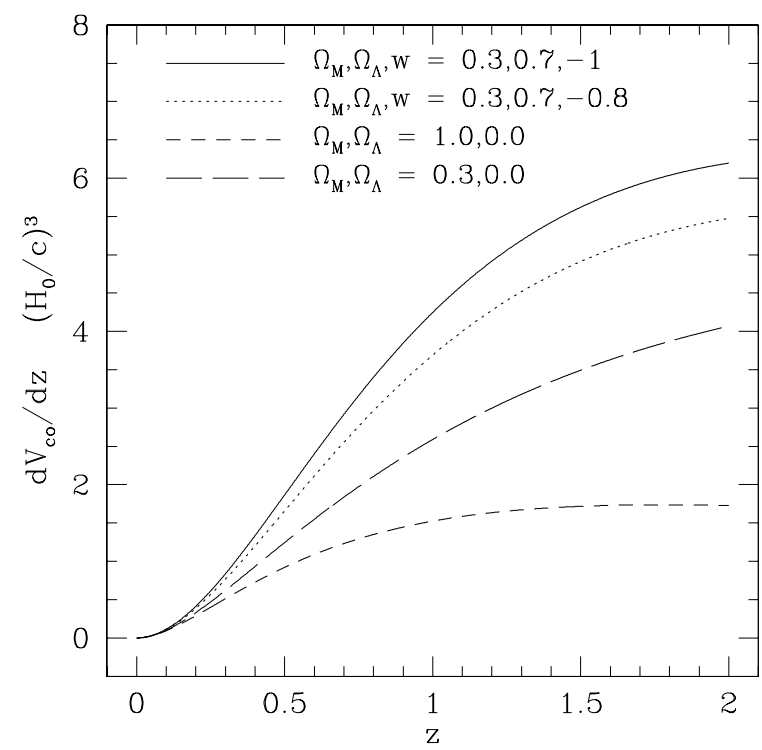

FIG. 3 Redshift dependence of comoving volume in various cosmologies. The quantity $d V_{\text {co }} / d z$ is the comoving volume of the entire sky between redshift $z$ and $z+d z$, divided by the redshift interval $d z$. If clusters were a non-evolving population of objects, one could distinguish between these cosmologies simply by counting the number of clusters on the sky in each redshift interval.

meaning that extended objects like clusters are far less bright at high redshifts.

It would be nice if $r_{\kappa}(z)$ could be expressed analytically for a general cosmology, but in most cases it cannot. However, a useful analytical expression for the divergence factor does exist for the case in which $\Omega_{\mathrm{R}}$ and $\Omega_{\Lambda}$ are negligible (Mattig 1958):

$$
r_{\kappa}(z)=\frac{2 c}{H_{0}} \frac{\Omega_{\mathrm{M}} z+\left(\Omega_{\mathrm{M}}-2\right)\left(\sqrt{1+\Omega_{\mathrm{M}} z}-1\right)}{\Omega_{\mathrm{M}}^{2}(1+z)} .
$$

Usually one needs to integrate equation (19) numerically and then insert the results into the $S_{\kappa}$ function to obtain the rest of the relations.

\section{Density Perturbations}

The very existence of galaxy clusters and the human beings who observe them demonstrates that the universe is not perfectly homogeneous. Therefore, the matter density in the early universe must have been slightly lumpy. At some early time these perturbations away from the mean density $\left\langle\rho_{M}\right\rangle$ correspond to an overdensity field

$$
\delta(\mathbf{x})=\frac{\rho_{M}(\mathbf{x})-\left\langle\rho_{M}\right\rangle}{\left\langle\rho_{M}\right\rangle}
$$

with Fourier components

$$
\delta_{\mathbf{k}}(k)=\int \delta(\mathbf{x}) e^{i \mathbf{k} \cdot \mathbf{x}} d^{3} x .
$$


In the plausible case that $\delta(\mathbf{x})$ is isotropic, it can be characterized by an isotropic power spectrum

$$
P(k) \equiv\left\langle\left|\delta_{k}\right|^{2}\right\rangle
$$

If $\delta(\mathbf{x})$ is also a Gaussian random field, then $P(k)$ is a complete statistical description of the initial perturbation spectrum.

The physical meaning of $P(k)$ becomes clearer if we assume it has a power-law form, with $P(k) \propto k^{n}$, and consider the variance in mass within identical volume elements corresponding to the length scale $k^{-1}$. For example, let $W(r)$ be a spherical window function that goes quickly to zero outside some characteristic radius $r_{W}$ and whose integral over all of space is unity. The mass perturbation smoothed over the window is

$$
\frac{\delta M}{M}(\mathbf{r})=\int \delta(\mathbf{x}) W(|\mathbf{x}-\mathbf{r}|) d^{3} x
$$

Using the convolution theorem, we can then write down the variance $\sigma^{2} \equiv\left\langle|\delta M / M|^{2}\right\rangle$ on this mass scale in terms of $W_{k}$, the Fourier transform of $W(r)$ :

$$
\sigma^{2}=\frac{1}{(2 \pi)^{3}} \int P(k)\left|W_{k}\right|^{2} d^{3} k .
$$

The variance in mass on scale $k$ for a power-law perturbation spectrum is therefore $\sigma^{2} \propto k^{n+3}$, because the windowing averages out modes with $k \gg r_{W}^{-1}$. Thus, the typical mass fluctuation on mass scale $M \propto k^{-3}$ is

$$
\frac{\delta M}{M} \propto M^{-\frac{n+3}{6}}
$$

Notice that large-scale homogeneity of the universe requires $n>-3$.

It is also illuminating to consider how $P(k)$ relates to fluctuations in the gravitational potential, $\delta \Phi \propto k \delta M$. The potential fluctuations owing to a power-law perturbation spectrum scale as $\delta \Phi \propto k^{(n-1) / 2}$. The magnitude of these fluctuations therefore diverges on either the high-mass end or the low-mass end, except in the case of $n=1$. This special property of the $P(k) \propto k$ power spectrum was noted independently by Harrison (1970), Peebles and Yu (1970), and Zeldovich (1972). Not only is this the most natural power-law spectrum, it also appears to be a good approximation to the true power spectrum of density fluctuations in the early universe. Inflationary models for the seeding of structure in the universe produce a Gaussian density field with a power-law index close to $n=1$ (Guth and Pi, 1982), which is consistent with the observed fluctuations in the cosmic microwave background (e.g., Spergel et al., 2003).

\section{Growth of Linear Perturbations}

Once the universe has been seeded with density perturbations they begin to grow because the gravity of slightly overdense regions attracts matter away from neighboring, slightly underdense regions. A complete treatment of perturbation growth is beyond the scope of this review, but some key features can be clarified with a simple toy model consisting of a uniform-density sphere that is slightly denser than its surroundings. The equation of motion for the radius $R$ of an expanding homogeneous sphere is analogous to the one governing the universe as whole. Integrating equation (9) with $a=R / R_{0}$, where $R_{0}$ is an arbitrary fiducial radius at which $\rho=\rho_{0}$, gives

$$
\frac{\dot{R}^{2}}{2}-\frac{4 \pi G \rho_{0} R_{0}^{3+3 w}}{3} R^{-(1+3 w)}=\epsilon .
$$

The constant of integration $\epsilon$ in this equation is again related to spatial curvature but can also be interpreted as the net specific energy of the sphere.

Now consider the behavior of two nearly identical spheres that both begin expanding from $R=0$ at $t=0$ but have specific energies that differ by a small amount $\delta \epsilon \ll \dot{R}^{2} / 2$. As these two spheres evolve, their radii will become slightly different by an amount $R_{2}-R_{1}=\delta R$, which satisfies the equation

$$
\int_{0}^{R} \frac{d R_{1}}{\dot{R}_{1}}=\int_{0}^{R+\delta R} \frac{d R_{2}}{\dot{R}_{2}} .
$$

In the linear regime, we can make the substitution $\dot{R}_{2}^{-1}=$ $\left(1-\dot{R}_{1}^{-2} \delta \epsilon\right) \dot{R}_{1}^{-1}$. If we then take the sphere of radius $R_{1}$ to be representative of the universe at large, we obtain

$$
\frac{\delta R}{R}=\frac{\delta \epsilon}{R_{0}^{2}} \frac{\dot{a}}{a} \int_{0}^{a} \frac{d a}{\dot{a}^{3}} .
$$

Because $\delta \rho / \rho=-3(1+w) \delta R / R$, this model leads to the following growth function for linear perturbations:

$$
D(a) \propto \frac{\delta \rho}{\rho} \propto \frac{\dot{a}}{a} \int_{0}^{a} \frac{d a}{\dot{a}^{3}},
$$

which is conventionally normalized so that $D(a)=1$ at $z=0$. Notice that the rate of perturbation growth implied by $D(a)$ does not depend on the scale of the perturbation, implying that density perturbations on all scales grow in unison.

This expression for the growth function is identical to those obtained through more rigorous arguments (e.g., Heath, 1977; Peebles, 1993). In a matter-dominated universe, perturbation amplitudes grow in proportion to the scale factor $a$. In a radiation-dominated universe, they grow $\propto a^{2}$. Handy numerical algorithms for computing $D(a)$ can be found in Hamilton (2001). A good approximation for the general case with a constant dark-energy density is

$$
\begin{aligned}
D(z)= & \frac{5 \Omega_{\mathrm{M}}(z)}{2(1+z)}\left\{\Omega_{\mathrm{M}}(z)^{4 / 7}-\Omega_{\Lambda}(z)\right. \\
& \left.+\left[1+\frac{\Omega_{\mathrm{M}}(z)}{2}\right]\left[1+\frac{\Omega_{\Lambda}(z)}{70}\right]\right\}^{-1}
\end{aligned}
$$


(see Carroll et al., 1992; Lahav et al., 1991).

If the dark-energy density is homogeneous but not constant in time, then the dark-energy density in the perturbed sphere of radius $R_{2}$ does not depend on its radius. In that case, one must solve a differential equation to determine the evolution of $\delta \equiv \delta \rho / \rho$ in the linear regime. Differentiating $R_{2}=R_{1}(1-\delta / 3)$ twice with respect to time and keeping only the lowest order terms leads to

$$
\ddot{\delta}+2 \frac{\dot{a}}{a} \dot{\delta}=4 \pi G \rho_{\mathrm{M}}(z) \delta
$$

in a universe with negligible radiation density. Wang and Steinhardt (1998) derive a useful approximation to the growth function by defining $\alpha_{w}$ such that

$$
\frac{d \ln \delta}{d \ln a}=\left[\Omega_{\mathrm{M}}(z)\right]^{\alpha_{w}} .
$$

For a slowly varying equation of state $\left(\left|d w / d \Omega_{\mathrm{M}}(z)\right| \ll\right.$ $\left.\left[1-\Omega_{\mathrm{M}}(z)\right]^{-1}\right)$, they find that

$\alpha_{w}=\frac{3}{5-w /(1-w)}+\frac{3}{125} \frac{(1-w)(1-3 w / 2)}{(1-6 w / 5)^{3}}\left[1-\Omega_{\mathrm{M}}(z)\right]$

to lowest order in $1-\Omega_{\mathrm{M}}(z)$. Using this expression for $\alpha_{w}$ in the integral

$$
D(a) \approx a \exp \left(\int_{a}^{1}\left\{1-\left[\Omega_{\mathrm{M}}(z)\right]^{\alpha_{w}}\right\} \frac{d a}{a}\right)
$$

reproduces the growth function obtained from numerical integration of equation (33) to better than $1 \%$ for $\Omega_{\mathrm{M}}(z)>0.2$.

These growth functions are valid only as long as pressure gradients do not alter the dynamics of the perturbation. Pressure effects are not an issue when the scale of a perturbation is larger than the Hubble length $c H^{-1}$. In that regime the growth functions found by solving equations (33) and (35) remain valid. Yet, as the universe ages, it encompasses perturbations of increasingly larger scale and additional physical effects enter the picture.

The bad news is that a variety of processes alter the scale-free nature of the original perturbation spectrum. The good news is that the imprint of these processes on $P(k)$ can tell us a great deal about the contents and dynamics of the universe. During the radiation-dominated era of the universe $\left(z>z_{\mathrm{eq}}\right)$, pressure effects begin to alter the growth of a given mode when its wavelength is finally contained within the horizon length $\sim c H^{-1}$. Then radiation pressure can effectively resist gravitational compression, inhibiting further growth of modes at that wavelength. Instead, these modes in the coupled photon-baryon fluid begin to oscillate as acoustic waves, and eventually damp owing to photon diffusion out of higher-density, higher-temperature regions. Perturbation growth in the dark-matter component therefore stalls near the amplitude at which the perturbations were first contained within the horizon because the gravitationally dominant photon component no longer spurs mode growth. These perturbations then resume growing at $z_{\text {eq }}$, when matter begins to dominate the dynamics. The transition from radiation domination to matter domination therefore imprints a bend in $P(k)$ on a length scale corresponding to the horizon scale at $z_{\text {eq }}$.

Perturbation growth is scale-independent during the matter-dominated era only insofar as the matter can be considered cold on the scale of the perturbation. If the characteristic velocities of the matter particles are not small compared to the escape velocity from the perturbation, then both pressure forces and particles streaming out of denser regions can damp small-scale perturbations. Each effect of this type imprints its own characteristic feature on $P(k)$.

All of these scale-imprinting effects that alter $P(k)$ from the time the primordial power spectrum is created until the present day are typically subsumed into a single quantity known as the transfer function, defined to be

$$
T(k) \equiv \frac{\delta_{k}(z=0)}{\delta_{k}(z) D(z)},
$$

where the symbol $k$ refers to comoving modes with wavenumber $(1+z) k$ in physical space, a convention implicit throughout this review. The redshift $z$ in this definition is assumed to be large enough that $\delta_{k}(z)$ reflects the original power spectrum imprinted by inflation or some other process. The transfer function therefore represents all the alterations of the original power spectrum that subsequently occur, except for those involving mode growth in the non-linear regime. If the primordial spectrum is a power law of index $n_{p} \approx 1$, then the power spectrum of linear perturbations at $z=0$ is $P(k) \propto k^{n_{p}} T^{2}(k)$.

\section{The CDM Power Spectrum}

The most successful models for the formation of largescale structures like clusters of galaxies assume that cold dark matter $(\mathrm{CDM})$ is responsible. Particles that interact only through gravity exert negligible pressure, and if their random velocities are small then they will not be able to escape from incipient potential wells on the scales of interest. That is, they will be too "cold" to damp the relevant perturbations by freely streaming out of them. Thus, the transfer function for a universe containing only radiation and cold dark matter has just one feature, corresponding to the wavenumber of the mode that enters the horizon at the matter-radiation equality redshift $z_{\text {eq }}$, with a comoving size $l_{\text {eq }} \sim c H_{0}^{-1}\left(\Omega_{\mathrm{M}} z_{\text {eq }}\right)^{-1 / 2} \sim$ $20\left(\Omega_{\mathrm{M}} h^{2}\right)^{-1} \mathrm{Mpc}$.

Growth of modes with smaller comoving wavelengths temporarily stalls from the redshift at which they enter the horizon until $z_{\text {eq }}$. Because radiation dominates the universe during this time interval, the comoving size of the horizon scales as $a$ while the growth function scales as $a^{2}$. Short-wavelength perturbations therefore miss out on a growth factor $\sim\left(k l_{\mathrm{eq}}\right)^{2}$, corresponding to the square 
of the change in scale factor from the time a perturbation enters the horizon to the time of matter-radiation equality. Growth of long-wavelength modes, on the other hand, does not stall at all. The behavior of the CDM transfer function in the two extremes is $T(k) \approx 1$ for $k \ll l_{\text {eq }}^{-1}$ and $T(k) \approx\left(k l_{\text {eq }}\right)^{-2}$ for $k \gg l_{\text {eq }}^{-1}$. For $n_{p}=1$, these scalings translate to $\delta M / M \sim M^{-2 / 3}$ on large scales and $\delta M / M \sim$ const. on small scales, meaning that structure formation in a CDM universe is hierarchical, with small-scale perturbations reaching the non-linear regime before larger-scale ones.

Numerical computations are needed to derive the exact CDM transfer function, but many authors have provided useful analytical fits to those numerical results. One such expression is

$$
\begin{array}{r}
T(k)=\frac{\ln (1+2.34 q)}{2.34 q}\left[1+3.89 q+(16.1 q)^{2}\right. \\
\left.+(5.46 q)^{3}+(6.71 q)^{4}\right]^{-1 / 4}
\end{array}
$$

with $q=k\left(\Omega_{\mathrm{M}} h^{2}\right)^{-1} \mathrm{Mpc}$ (Bardeen et al., 1986). Allowing for trace populations of baryons and massive neutrinos alters the CDM power spectrum in minor but interesting ways. For example, a small proportion of baryons lowers the apparent dark-matter density parameter, causing a shape-preserving shift in the CDM transfer function (Peacock and Dodds, 1994). This shift can be reproduced by setting $q=k(\Gamma h)^{-1} \mathrm{Mpc}$, so that it includes a shape parameter $\Gamma=\Omega_{\mathrm{M}} h \exp \left[-\Omega_{\mathrm{b}}(1+\right.$ $\left.\sqrt{2 h} / \Omega_{\mathrm{M}}\right)$ ] (Sugivama, 1995). Fitting formulae accomodating additional modifications owing to baryons and massive neutrinos can be found in Eisenstein and $\mathrm{Hu}$ (1998, 1999).

\section{Power Spectrum Normalization}

The preceding sections give the theoretical expectations for the shape and growth rate of the density perturbation spectrum but do not specify its normalization. Because inflationary theories do not make firm predictions about the amplitude of the primordial power spectrum, the normalization of $P(k)$ must be determined observationally. For example, measurements of the present-day mass distribution of the universe indicate that $\delta M / M \approx 1$ within comoving spheres of radius $8 h^{-1} \mathrm{Mpc}$ (Sec. III.C), as suggested by early galaxy surveys showing that the variance in galaxy counts was of order unity on this length scale (Davis and Peebles, 1983).

This feature of the universe is the motivation for expressing the power-spectrum normalization in terms of the quantity $\sigma_{8}$, where

$$
\sigma_{8}^{2}=\frac{1}{(2 \pi)^{3}} \int P(k)\left|W_{k}\right|^{2} d^{3} k
$$

is the variance defined with respect to a top-hat window function $W(r)$ having a constant value inside a comoving radius of $8 h^{-1} \mathrm{Mpc}$ and vanishing outside this radius. When using this formula, one must keep in mind that $P(k)$ refers to the power spectrum of linear perturbations evolved to $z=0$ according to the growth function $D(z)$, which is valid only for small perturbations. There are other ways of characterizing the power-spectrum normalization, but $\sigma_{8}$ is the most widely-used parameter.

\section{Summary of Cosmological Parameters}

At the beginning of this recipe, we promised to encapsulate the overall cosmological model in two small sets of parameters. The set governing the global behavior of the universe consists of $H_{0}, \Omega_{\mathrm{M}}, \Omega_{\mathrm{b}}, \Omega_{\mathrm{R}}, \Omega_{\Lambda}$, and $w$. The set governing the initial density perturbation spectrum consists of $\sigma_{8}$ and $n_{p}$. The shape parameter $\Gamma$ is not a free parameter in standard cold dark matter models but is sometimes treated as a free parameter in order to test variants of the standard model.

In the concordance model, also known as the $\Lambda \mathrm{CDM}$ model, to denote cold dark matter with a cosmological constant, these parameters are all assigned values close to the most likely values implied by observations:

- Hubble's constant. The consensus value of this parameter, measured primarily from the expansion rate of the local universe is $H_{0}=71 \pm$ $7 \mathrm{~km} \mathrm{~s}^{-1} \mathrm{Mpc}^{-1}$ (Freedman et al., 2001).

- Matter density. Several different methods involving clusters indicate that $\Omega_{\mathrm{M}} \approx 0.3$ (Sec. III.C Sec. III.D.3). Combining the results of distant supernova observations and observations of temperature patterns in the microwave background gives a similar value for this parameter. Figure 4 shows one example of these mutual constraints in the $\Omega_{\mathrm{M}^{-}}$ $\Omega_{\Lambda}$ plane.

- Baryon density. The abundances of light elements formed during primordial nucleosynthesis indicate that $\Omega_{\mathrm{b}}=0.02 h^{-2}$, equal to $\Omega_{\mathrm{b}}=0.04$ for the value of Hubble's constant given above (e.g., Burles et al., 2001). This value is consistent with the baryon density inferred from the fluctuations in the cosmic microwave background (e.g., Spergel et al., 2003).

- Radiation density. The energy density $\Omega_{\mathrm{R}}$ in electromagnetic radiation is simply calculated from the microwave background temperature $T_{\mathrm{CMB}}=$ $2.728 \pm 0.004$ (Fixsen et al., 1996) and Hubble's constant. Neutrinos may also contribute to the energy density in relativistic matter, if their masses are sufficiently small, but this contribution is currently too small to affect the global dynamics.

- Dark energy density. Observations of distant supernovae imply that the expansion of the universe is accelerating at a rate consistent with a constant 
dark-energy density corresponding to $\Omega_{\Lambda} \approx 0.7$ (Perlmutter et al., 1999; Riess et al., 1998, 2004). Combining the matter density inferred from clusters with the flat geometry inferred from temperature patterns in the microwave background corroborates this result (e.g., Bahcall et al., 1999, see also Figure 4).

- Dark energy equation of state. Observations of microwave background patterns, when combined with observations of large-scale structure are consistent with Einstein's cosmological constant $(w=-1.0)$ but not with $w \gtrsim-0.8$ (Spergel et al., 2003). Alternatively, combining cluster surveys with observations of distant supernovae leads to similar constraints, $w=-0.95_{-0.35}^{+0.30}$ (Schuecker et al., 2003). However, theoretical arguments suggest that the parameter $w$ may be redshift dependent (Peebles and Ratra, 2003).

- Normalization of density perturbations. The cluster observations discussed in Sec.III.Cland Sec.III.D.3 indicate that the power-spectrum normalization falls into the range $\sigma_{8} \approx 0.7-1.0$. This range is consistent with both structures in the cosmic microwave background and other observations of large-scale structure.

- Slope of primordial perturbation spectrum. All available information indicates that $n_{p} \approx 1$. Constraints from observations of the microwave background, when combined with optical observations of large-scale structure, give $n_{p}=0.97 \pm 0.03$ (Spergel et al., 2003).

- Shape parameter of perturbation spectrum. The concordance values of $\Omega_{\mathrm{M}}, \Omega_{\mathrm{b}}$, and $H_{0}$ given above imply $\Gamma \approx 0.2$, which agrees with the value of $\Gamma$ derived from observations of large-scale structure (e.g., Peacock and Dodds, 1994; Schuecker et al., 2001; Szalav et al., 2003), an important element of self-consistency in the concordance model.

Several other closely related models have been pursued during the last two decades, but none of them have proven as successful in explaining such a large number of observations. Here are a few variants some of which will be discussed later in connection with the parameter constraints derived from cluster surveys:

- Standard cold dark matter (SCDM). In this model, the universe is assumed to be flat, with no dark energy, so $\Omega_{\mathrm{M}}=1$ and $\Omega_{\Lambda}=0$. For this value of the matter density, measurements of large-scale structure, including clusters, imply that $\sigma_{8}=0.4-0.5$ (Sec. III.C). However, the shape parameter implied for this model, given the observed Hubble constant, is $\Gamma \approx 0.7$, which conflicts with observations of large-scale structure (e.g., Szalav et al.,

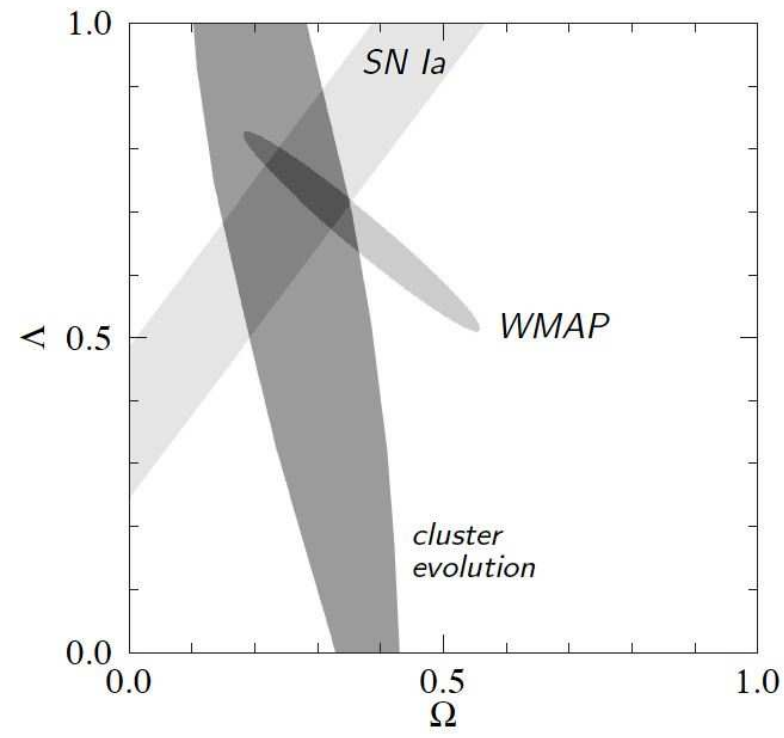

FIG. 4 Cosmological constraints from cluster evolution (Vikhlinin et al., 2003), supernovae (Perlmutter et al., 1999; Riess et al., 1998), and WMAP observations of the cosmic microwave background (Spergel et al., 2003). The horizontal axis labeled $\Omega$ gives the value of $\Omega_{\mathrm{M}}$; the vertical axis labeled $\Lambda$ gives the value of $\Omega_{\Lambda}$. These particular contraints from cluster evolution are based on the baryonic mass function of clusters (Sec.III.D.3), but other measures of cluster evolution give similar results. The complementarity of these constraints is evident from the figure, and the common region of overlap near $\Omega_{\mathrm{M}}=0.3$ and $\Omega_{\Lambda}=0.7$ is reassuring evidence of consistency in the overall picture. (Figure courtesy of Alexey Vikhlinin.)

2003). Also, this value of $\Omega_{\mathrm{M}}$ leads to a baryon-todark-matter ratio $f_{b}=\Omega_{\mathrm{b}} / \Omega_{\mathrm{M}}$ that is inconsistent with cluster observations (Sec. III.C.7).

- Tilted cold dark matter. One way to make models with $\Omega_{\mathrm{M}}=1$ more consistent with large-scale structure observations is to assume that the primordial perturbation spectrum is "tilted" so that it is significantly shallower than $n_{p}=1$ (e.g., Cen et al., 1992). However, such models conflict with the strong constraints on $n_{p}$ inferred from microwave background observations.

- Ad hoc power spectrum ( $\tau \mathrm{CDM})$. Another option for making $\Omega_{\mathrm{M}}=1$ models more consistent with observations is to arbitrarily adjust the shape of the perturbation spectrum to fit the observations. One such realization is the $\tau \mathrm{CDM}$ model (Jenkins et al., 1998), which sets $\Omega_{\mathrm{M}}=1, \sigma_{8}=0.5$ and $\Gamma=0.2$, even though there is little physical justification for having such a low value of $\Gamma$ in a cosmology with such a large matter density (but see White et al., 1995).

- Open cold dark matter (OCDM). There is also the option of accepting the evidence that $\Omega_{\mathrm{M}} \approx 0.3 \mathrm{but}$ 
dispensing with dark energy $\left(\Omega_{\Lambda}=0\right)$ so that the universe has an open geometry. In this case, the perturbation spectrum can be identical to the one in the $\Lambda \mathrm{CDM}$ concordance model, but the growth rate of those perturbations differs because of the changed expansion rate at late times.

This review does not consider models involving forms of dark matter other than cold dark matter, but it does consider models with generalized forms of dark energy having $w \neq-1$ ( $w \mathrm{CDM})$. Current cluster surveys are not yet large enough to place strong constraints on this equation-of-state parameter but they might provide much stronger constraints in the coming decade (Sec. III.D.4).

\section{B. Cluster Formation}

Cluster formation from perturbations in the density distribution of cold dark matter is a hierarchical process. Small subclumps of matter are the first pieces of the cluster to deviate from the Hubble flow and undergo gravitational relaxation because the density perturbations have larger amplitudes on smaller mass scales, These small pieces then merge and coalesce to form progressively larger structures as perturbations on larger mass scales reach the non-linear regime. A full understanding of the details of this hierarchical merging process requires numerical simulations, but simplified, spherically symmetric models of cluster formation illustrate many of the important concepts. This part of the review shows how a cluster would grow from a spherically symmetric mass perturbation and then refines the details of that simplified approach, based on what we have learned from numerical simulations.

\section{Spherical Collapse}

The most basic features of cluster formation can be understood in terms of a spherically symmetric collapse model (e.g., Bertschinger, 1985; Fillmore and Goldreich, 1984; Gunn and Gott, 1972). In such a model, the matter that goes on to form a cluster begins as a low-amplitude density perturbation that initially expands along with the rest of the universe. The perturbation's gravitational pull gradually slows the expansion of that matter, eventually halting and reversing the expansion. A cluster of matter then forms at the center of the perturbation, and the rate at which additional matter accretes onto the cluster depends on the distribution of density with radius in the initial perturbation.

In a geometry that is perfectly spherically symmetric, the behavior of an individual mass shell in the presence of a homogeneous generalized dark-energy field follows the equation of motion

$$
\ddot{r}_{\mathrm{sh}}=-\frac{G M_{\mathrm{sh}}}{r_{\mathrm{sh}}^{2}}-\frac{1+3 w}{2} \Omega_{\Lambda} H_{0}^{2}(1+z)^{3(1+w)} r_{\mathrm{sh}},
$$

where $r_{\mathrm{sh}}$ is the shell radius and $M_{\mathrm{sh}}$ is the mass enclosed within $r_{\mathrm{sh}}$. Throughout the early evolution of a spherical perturbation, the value of $M_{\mathrm{sh}}$ within a given mass shell remains constant. Thus, if the dark-energy term is negligible, the radius of a mass shell obeys the parametric solution $r_{\mathrm{sh}}=r_{\mathrm{ta}}\left[\left(1-\cos \theta_{M}\right) / 2\right], t=t_{\mathrm{c}}\left[\left(\theta_{M}-\sin \theta_{M}\right) / 2 \pi\right]$, with a turnaround radius $r_{\mathrm{ta}}=\left[\left(2 G M_{\mathrm{sh}} t_{\mathrm{c}}^{2}\right) / \pi^{2}\right]^{1 / 3}$ for a shell that collapses to the origin at time $t_{\mathrm{c}}$. The solution for $\Omega_{\Lambda} \approx 0.7$ and $w \approx-1$ is not much different because the dark-energy term remains $\lesssim 15 \%$ of the matter term during the trajectory of all shells that collapse to the origin by the present time. If greater accuracy is needed, a shell's trajectory can be computed numerically from equation (42). ${ }^{4}$

Once a shell collapses, the mass within it no longer remains constant. Because the dark matter within a collapsing shell is collisionless, shells on different trajectories can easily interpenetrate. The radii of collapsed shells in this idealized geometry therefore oscillate symmetrically about the origin, and the amplitudes of these oscillations modestly decrease with time as mass associated with other collapsed shells accumulates within the oscillations' turning points (Gunn, 1977).

The accretion process in real clusters is not so symmetric. Instead, gravitational forces between infalling clumps of matter produce a time-varying gravitational potential that randomizes the velocities of the infalling particles, yielding a Maxwellian velocity distribution in which temperature is proportional to the particle mass. This process, known as "violent relaxation" (Lvnden-Bell, 1967), leads to a state of virial equilibrium in which the total kinetic energy $E_{K}$ is related to the total gravitational potential energy $E_{G}$ through the equation

$$
E_{G}+2 E_{K}=4 \pi P_{\mathrm{b}} r_{\mathrm{b}}^{3}
$$

where $P_{\mathrm{b}}$ is the effective pressure owing to infalling matter at the boundary $r_{\mathrm{b}}$ of the collapsed system (Sec. II.A.2). Setting $P_{\mathrm{b}}$ to zero yields the usual form of the virial theorem for gravitationally bound systems.

A common toy model for estimating the location of a cluster's outer boundary is the spherical top-hat model, which assumes that the perturbation leading to a cluster is a spherical region of constant density. All of the mass shells in such a perturbation move in unison and collapse to the origin simultaneously. The virial theorem therefore suggests that the bounding radius of the cluster after it collapses and relaxes should be in the neighborhood of half the turnaround radius. Numerical simulations indeed show that particle velocities within this radius are

\footnotetext{
${ }^{4}$ Here we are making the standard assumption that the collapsing dark matter has no effect on the local dark-energy density (e.g. Wang and Steinhardt, 1998; Weinberg and Kamionkowski, 2003). If in fact the dark-matter collapse alters the local properties of dark energy, the dynamics could be somewhat altered (Mota and van de Bruck, 2004).
} 
generally isotropic and those outside this radius are generally infalling, but the boundary between the isotropic and infalling regions is not particularly distinct Evrard, 1990; Navarro and White, 1993).

The spherical top-hat model has actually led to several different definitions for the virial radius of a cluster. If one assumes that all the mass in the original top-hat perturbation ends up within $r_{\mathrm{ta}} / 2$, then the mass density in that region is $6 M / \pi r_{\mathrm{ta}}^{3}$. In a matterdominated universe with zero dark energy, this density is equal to $\Delta_{\mathrm{v}}=8 \pi^{2} /(H t)^{2}$ times the critical density $\rho_{\text {cr }} \equiv 3 H^{2} / 8 \pi G$. Thus, for a flat, matter-dominated universe in which $H t=2 / 3$, the mean density of a perturbation that has just collapsed is taken to be $18 \pi^{2} \approx 178$ times the critical density. A useful approximation for $\Delta_{\mathrm{V}}$ in a flat universe with a non-zero cosmological constant $(w=-1)$ is

$$
\Delta_{\mathrm{v}}=18 \pi^{2}+82\left[\Omega_{\mathrm{M}}(z)-1\right]-39\left[\Omega_{\mathrm{M}}(z)-1\right]^{2}
$$

(Brvan and Norman, 1998). Because the outer radius of a real cluster is not distinct, one pragmatic definition of the virial radius is then the radius $r_{\mathrm{V}}$ within which the mean matter density is $\Delta_{\mathrm{v}} \rho_{\mathrm{cr}}$ (Eke et al., 1996). However, the numerical value of $\Delta_{\mathrm{v}}$ in a flat, matterdominated universe has inspired other definitions. A common alternative is the scale radius $r_{200}$, within which the mean matter density is $200 \rho_{\mathrm{cr}}$. Another frequently used scale radius is $r_{180 \mathrm{~m}}$, within which the mean matter density is 180 times the mean background density $\Omega_{\mathrm{M}}(z) \rho_{\mathrm{cr}}$. As long as $\Omega_{\mathrm{M}}(z) \approx 1$, both of these scale radii are nearly identical to $r_{\mathrm{v}}$, but because $\Omega_{\mathrm{M}} \approx 0.3$ at the present time, these radii are now somewhat different, with $r_{200}<r_{\mathrm{v}}<r_{180 \mathrm{~m}}$. This multiplicity of definitions for the radius of a cluster is a potential source of confusion, but as we will see below, each of these scale radii can be particularly well suited to certain applications.

\section{Cluster Mass Profiles}

Observations of galaxy clusters have long indicated that the velocity dispersion of a cluster's galaxies remains relatively constant with distance from the cluster's center, implying an underlying mass-density profile $\rho_{\mathrm{M}}(r) \propto r^{-2}$. The simplest analytical cluster model consistent with such a density profile is the singular isothermal sphere, in which the velocity dispersion $\sigma_{v}$ is constant and isotropic at every point and $\rho_{\mathrm{M}}(r)=\sigma_{v}^{2} / 2 \pi G r^{2}$ (e.g., Binnev and Tremaine, 1987). This model is useful for making analytical estimates of cluster properties, but it is incomplete because the total mass diverges linearly with radius.

Numerical simulations of cluster formation produce dark-matter halos whose density profiles are shallower than isothermal at small radii and steeper than isothermal at large radii. A generic form for representing these profiles is

$$
\rho_{\mathrm{M}}(r) \propto r^{-p}\left(r+r_{s}\right)^{p-q},
$$

where the parameters $p$ and $q$ describe the inner and outer power-law slopes and the radius $r_{s}$ specifies where the profile steepens. Groups that have fit such profiles to simulated clusters disagree about the best values of $p$ and $q$ but typically find $1 \lesssim p \lesssim 1.5$ and $2.5 \lesssim q \lesssim 3$. Specific examples include the NFW profile, with $p=1$ and $q=3$ (Navarro et al., 1997), the Moore profile, with $p=1.5$ and $q=3$, and the Rasia et al. (2003) profile, with $p=1$ and $q=2.5$. Both optical and X-ray observations indicate that density profiles of this sort are good representations of the underlying mass profiles of clusters, at least outside of the innermost regions (Carlberg et al., 1997b; Lewis et al., 2003; Pratt and Arnaud, 2002). Observing the asymptotic inner slope $p$ is currently a matter of great observational interest, as the cuspiness of dark-matter density profiles at $r=0$ is one of the acid tests of the CDM paradigm for structure formation (see Navarro et al., 2003, and references therein). However, we will not discuss that issue here because the global properties of clusters depend little on the value of $p$. In this review, we will use the NFW profile when necessary because it remains thee most widely used fitting formula for representing the results of cosmological simulations.

The transition of the density profile from shallow to steep can also be expressed in terms of a concentration parameter $c=r_{\mathrm{b}} / r_{s}$, which expresses the bounding radius of the cluster in units of $r_{s}$. Because the concentration parameter depends on $r_{\mathrm{b}}$, numerical values of $c$ depend somewhat on whether the bounding radius is taken to be $r_{\mathrm{v}}, r_{200}, r_{180 \mathrm{~m}}$, or something similar. However, these radii are not vastly different because they are generally several times larger than $r_{s}$, meaning that the enclosed mass is not rapidly diverging in the neighborhood of the virial radius. Typical concentration parameters for simulated clusters are in the range $c \sim 4-10$, with a scatter in $\ln c$ of 0.2-0.35 (Jing, 2000). Also, lower-mass objects tend to have higher halo concentrations because they formed earlier in time, when the overall density of the universe was greater (Bullock et al., 2001; Eke et al., 2001; Navarro et al., 1997).

\section{Defining Cluster Mass}

Even with these more sophisticated forms for the density profile, mass still diverges with radius. Thus, a cluster's mass and all the relations linking that mass to other observable quantities depend on how one chooses to define a cluster's outer boundary. One would like to define that boundary so as to maximize the simplicity of the relationships between cluster mass and other observables, but no single definition is best for all applications.

The easiest way to link observations to theoretical models is through definitions taking the mass of a cluster to be $M_{\Delta}$, the amount of matter contained in a spherical region of radius $r_{\Delta}$ whose mean density is $\Delta \cdot \rho_{\mathrm{cr}}$. It is also common for cluster mass to be defined with respect to the background mass density, so that the mean density of 
matter within the virial radius is $\Delta \cdot \Omega_{\mathrm{M}}(z) \rho_{\mathrm{cr}}$, but applying this definition to observations requires prior knowledge of $\Omega_{\mathrm{M}}$. Spherical top-hat collapse suggests that $\Delta_{\mathrm{V}}$ is a good choice for the density threshold. However, observers often prefer to raise that threshold to $\Delta=200$ or even $\Delta=500$ for two reasons. The properties of a cluster are easier to observe in regions where the density contrast is higher, and simulations show that the region within $r_{500}$ is considerably more relaxed than the region within $r_{\mathrm{v}}$.

As an example of such definitions in action, consider the relation between velocity dispersion and the virial mass $M_{\mathrm{v}}$ obtained by truncating a singular isothermal sphere at the virial radius $r_{\mathrm{v}}$ :

$$
M_{v}=f_{\sigma} \frac{4 \sigma_{v}^{3}}{G H \Delta_{\mathrm{v}}^{1 / 2}},
$$

where the factor $f_{\sigma}$ is a parameter that can be adjusted to account for the fact that clusters are not perfect isothermal spheres (Brvan and Norman, 1998; Eke et al., 1996; Evrard, 1989). The presence of this parameter is a reminder that the derivation of this relation should not be taken too literally. Truncation of the mass distribution at $r_{\Delta}$ formally implies a non-zero boundary pressure that shifts the virial relation for this configuration so that $E_{K}=-3 E_{G} / 4$, which is inconsistent with the definition of $r_{\mathrm{v}}$ (Voit, 2000). This functional form for the $M_{\mathrm{v}}-\sigma_{v}$ is useful primarily as a fitting formula that accounts for most of the cosmology-dependent changes in the normalization of the relation. However, because the density profiles of dark matter halos defined in this way depend on both mass and redshift, the correction factor $f_{\sigma}$ is not a universal constant.

Recent work has shown that defining a cluster's mass using the threshold $\Delta=200$ leads to an $M_{200} \sigma_{v}$ relation that is remarkably independent of cosmology. Evrard (2004) finds that the relation

$$
M_{200}=\frac{10^{15} h^{-1} M_{\odot}}{H / H_{0}}\left(\frac{\sigma_{v}}{1080 \mathrm{~km} \mathrm{~s}^{-1}}\right)^{3}
$$

is an excellent fit to a wide range of simulated clusters sampled over a wide range of redshifts. This relation is equivalent to setting $f_{\sigma}=1.2$ and $\Delta_{\mathrm{V}}=200$ in equation (46).

Conversions between $M_{\mathrm{v}}$ and $M_{\Delta}$ defined with respect to an arbitrary $\Delta$ are straightforward as long as a cluster's concentration parameter is known. From the definitions of these masses, we have

$$
\frac{M_{\Delta}}{M_{\mathrm{v}}}=\frac{\Delta}{\Delta_{\mathrm{v}}}\left(\frac{r_{\Delta}}{r_{\mathrm{v}}}\right)^{3}
$$

and the halo concentration gives the relationship between $r_{\Delta}$ and $r_{\mathrm{v}}$. $\mathrm{Hu}$ and Kravtsov (2003) have provided a useful approximation for this relation in the case of an NFW profile. Recasting their formulae in slightly different notation, one can write the halo concentration $c_{\Delta}$ defined with respect to $r_{\Delta}$ in terms of the concentration $c_{\mathrm{V}}$ defined with respect to $r_{\mathrm{v}}$ :

$$
c_{\Delta}=\frac{1}{\left[a_{1} f_{c}^{2 p_{c}}+\left(\frac{3}{4}\right)^{2}\right]^{-1 / 2}+2 f_{c}},
$$

with $f_{c}=\left(\Delta / c_{\mathrm{v}}^{3} \Delta_{\mathrm{v}}\right)\left[\ln \left(1+c_{\mathrm{v}}\right)-c_{\mathrm{v}} /\left(1+c_{\mathrm{v}}\right)\right], p_{c}=a_{2}+$ $a_{3} \ln f_{c}+a_{4}\left(\ln f_{c}\right)^{2}$ and $\left(a_{1}, \ldots, a_{4}\right)=(0.5116,-0.4283$, $\left.-3.13 \times 10^{-3},-3.52 \times 10^{-5}\right)$. Plugging the ratio $r_{\Delta} / r_{\mathrm{v}}=$ $c_{\Delta} / c_{\mathrm{v}}$ given by this approximation into equation (48) converts cluster masses with an accuracy $\sim 0.3 \%$ for the halo concentrations typical of clusters.

Some other definitions of cluster mass are useful in certain contexts but are more difficult to relate to the tophat collapse model. For example, observers who measure cluster mass using gravitational lensing or the total optical luminosity are essentially measuring cluster mass within a cylinder along the line of sight rather than a sphere. In principle, these observables can be linked with cluster masses defined with respect to a cylindrical boundary, but the relationships between those cylindrical masses and models of structure formation are not as well understood as their spherical counterparts. On the theoretical side, the masses of clusters identified in numerical simulations are sometimes defined using a "friends-offriends" algorithm that links neighboring mass particles (e.g., Davis et al., 1985). However, clusters defined in this way often have irregular boundaries (White, 2001), making this sort of definition difficult to apply to observations. Masses defined within spheres also have their shortcomings, particularly in cases where two clusters are just beginning to merge, but in general provide the most direct link between cosmological models and observations.

\section{Cluster Mass Function}

Some of the most powerful constraints on current cosmological models come from observations of how clusters evolve with time. Because cosmological time scales are so long, we cannot observe how individual clusters evolve but rather observe how the demographics of the entire cluster population changes with redshift. A important conceptual tool in this effort is the cluster mass function, $n_{M}(M)$ which gives the number density of clusters with mass greater than $M$ in a comoving volume element. Notice that the cluster mass function inevitably depends on how one defines cluster mass.

Combining spherical top-hat collapse with the growth function for linear perturbations has led to a widely used semi-analytical method for expressing the cluster mass function in terms of cosmological parameters. Press and Schechter (1974) pioneered the basic approach, which was refined and extended by Bond et al. (1991), Bower (1991), and Lacev and Cole (1993). This class of models simplifies the problem of structure formation by assuming that all density perturbations continue to grow 
according to the linear growth rate $D(z)$ even when their amplitudes become non-linear. When perturbations are treated in this way, their variance on mass scale $M$ as a function of redshift is

$$
\sigma^{2}(M, z)=\frac{D^{2}(z)}{(2 \pi)^{3}} \int P(k)\left|W_{k}(M)\right|^{2} d^{3} k,
$$

where $W_{k}(M)=3\left(\sin k r_{M}-k r_{M} \cos k r_{M}\right) /\left(k r_{M}\right)^{3}$ with $r_{M}=\left(3 M / 4 \pi \Omega_{\mathrm{M}} \rho_{\mathrm{cr} 0}\right)^{1 / 3}$ is the Fourier-space representation of a top-hat window function that encloses mass $M$. The normalization of $P(k)$ is set so that $\sigma\left(M_{8}, 0\right)=\sigma_{8}$ for $M_{8} \equiv\left(8 h^{-1} \mathrm{Mpc}\right)^{3} H_{0}^{2} \Omega_{\mathrm{M}} / 2 G=6.0 \times 10^{14} \Omega_{\mathrm{M}} h^{-1} M_{\odot}$. These perturbations are then assumed to collapse and virialize when their density contrast $\delta=\delta \rho / \rho$ exceeds a critical threshold $\delta_{c}$.

Suppose the initial density perturbations are gaussian with a variance $\sigma^{2}(M, z)$ that declines monotonically with mass. Then according to the Press-Schechter approach, the probability that a region of mass $M$ exceeds the collapse threshold at redshift $z$ is $\operatorname{erfc}\left[\delta_{c} / \sqrt{2} \sigma(M, z)\right]$, where erfc $(x)$ is the complementary error function. Implicit in this expression is the notion that all the mass in the universe belongs to collapsed, virialized objects when viewed on sufficiently small mass scales. It then follows that the cluster mass function on scale $M$ at redshift $z$ is

$$
n_{M}(M, z)=\frac{\Omega_{\mathrm{M}} \rho_{\mathrm{cr} 0}}{M} \operatorname{erfc}\left[\frac{\delta_{c}}{\sqrt{2} \sigma(M, z)}\right] .
$$

This expression implies that the shape of the mass function depends only on $\sigma(M, z)$ and remains invariant with respect to the characteristic collapsing mass scale $M_{*}(z)$ at which $\sigma\left(M_{*}, z\right)=\delta_{c}$. Observers often work with the mass function in a differential form, such as $d n_{M} / d \ln M$, but theorists prefer expressing the differential form in terms of the shape-governing function $\sigma(M, z)$. Then the differential mass function takes the form

$$
\frac{d n_{M}}{d \ln \sigma^{-1}}=\sqrt{\frac{2}{\pi}} \frac{\Omega_{\mathrm{M}} \rho_{\mathrm{cr} 0}}{M} \frac{\delta_{c}}{\sigma} \exp \left[-\frac{\delta_{c}^{2}}{2 \sigma^{2}}\right] .
$$

Both of these forms for the mass function can be straightforwardly extended to cases in which the perturbations are non-gaussian (e.g., Robinson et al., 2000).

The value of the critical threshold $\delta_{c}$ was originally inferred from spherical top-hat collapse. Expanding the parametric solution for spherical collapse in powers of $\theta_{M}$ leads to the following relation at early times:

$$
r_{\mathrm{sh}} \approx\left(\frac{9 G M t^{2}}{2}\right)^{1 / 3}\left[1-\frac{1}{20}\left(\frac{12 \pi t}{t_{\mathrm{c}}}\right)^{2 / 3}\right]
$$

The leading term in this expression characterizes the behavior of a critical-density sphere and the second term describes how the evolution of a slightly overdense sphere deviates from that of a critical-density sphere. Assuming that this deviation grows according to equation (53) until the moment of collapse and virialization $\left(t=t_{\mathrm{c}}\right)$ gives the value of the critical threshold in a flat universe with $\Omega_{\mathrm{M}}=1: \delta_{c}=3(12 \pi)^{2 / 3} / 20 \approx 1.686$. Generalizing this treatment to cases where $\Omega_{\mathrm{M}} \neq 1$ produces only minor differences in $\delta_{c}$ for interesting values of the cosmological parameters (Eke et al., 1996; Lacev and Cole, 1993).

The preceding derivation of the cluster mass function is not terribly rigorous, but it is useful because adopting $\delta_{c}=1.686$ leads to mass functions that agree reasonably well with those derived from numerical simulations. Treating perturbation collapse as ellipsoidal rather than spherical improves that agreement (Sheth et al., 2001). Sheth and Tormen (1999) have shown that the expression

$$
\begin{aligned}
\frac{d n_{M}}{d \ln \sigma^{-1}}=A_{\mathrm{s}} & \sqrt{\frac{2 a_{\mathrm{s}}}{\pi}}\left[1+\left(\frac{\sigma^{2}}{a_{\mathrm{s}} \delta_{c}^{2}}\right)^{p_{\mathrm{s}}}\right] \\
& \times \frac{\Omega_{\mathrm{M}} \rho_{\mathrm{cr} 0}}{M} \frac{\delta_{c}}{\sigma} \exp \left[-\frac{a_{\mathrm{s}} \delta_{c}^{2}}{2 \sigma^{2}}\right],
\end{aligned}
$$

with $A_{\mathrm{s}}=0.3222, a_{\mathrm{s}}=0.707$, and $p_{\mathrm{s}}=0.3$ is quite an accurate representation of the mass functions from several different numerical simulations. However, because semi-analytical mass functions like these are not rigorously derived, they are essentially just fitting formulae that conveniently express the simulation results and should treated cautiously outside the cosmological models against which they have been tested.

A particularly well-tested fitting formula for cluster mass functions has been provided by Jenkins et al. (2001). Combining results for simulated clusters spanning a mass range from $<10^{12} M_{\odot}$ to $>10^{15} M_{\odot}$ and sampled at a number of different redshifts, they found that the form of $d n_{M} / d \ln \sigma^{-1}$ was nearly invariant if they defined cluster mass to be $M_{180 \mathrm{~m}}$, the mass within a sphere of mean density $180 \Omega_{\mathrm{M}}(z) \rho_{\mathrm{cr}}$. When this definition of cluster mass is used, the formula

$$
\frac{d n_{M}}{d \ln \sigma^{-1}}=A_{\mathrm{J}} \frac{\Omega_{\mathrm{M}} \rho_{\mathrm{cr} 0}}{M} \exp \left[-\left|\ln \sigma^{-1}+B_{\mathrm{J}}\right|^{\epsilon_{\mathrm{J}}}\right]
$$

with $A_{\mathrm{J}}=0.301, B_{\mathrm{J}}=0.64$, and $\epsilon_{\mathrm{J}}=3.82$, reproduces the cluster mass function to $\sim 20 \%$ accuracy for all the cosmologies tested, including $\Lambda \mathrm{CDM}, \tau \mathrm{CDM}$, and OCDM. In this expression, $A_{\mathrm{J}}$ governs the fraction of the total mass in collapsed objects, $e^{B_{\mathrm{J}}}$ functions as a collapse threshold analogous to $\delta_{c}$, and $\epsilon_{\mathrm{J}}$ stretches the mass function to fit the simulations. The Sheth-Tormen mass function of equation (54) fits these same numerical simulations nearly as well.

The exponential sensitivity to mass and redshift evident in these expressions for the cluster mass function is both a blessing and a curse. On the one hand, it makes cluster counts and their evolution with redshift a very powerful probe of cosmological parameters. Figure 5 showing the cluster mass function and its evolution with time for five different cosmologies, illustrates how sensitive mass-function evolution is to the matter density. On the other hand, any systematic errors in the measurement of cluster mass, including inconsistencies in the definition 


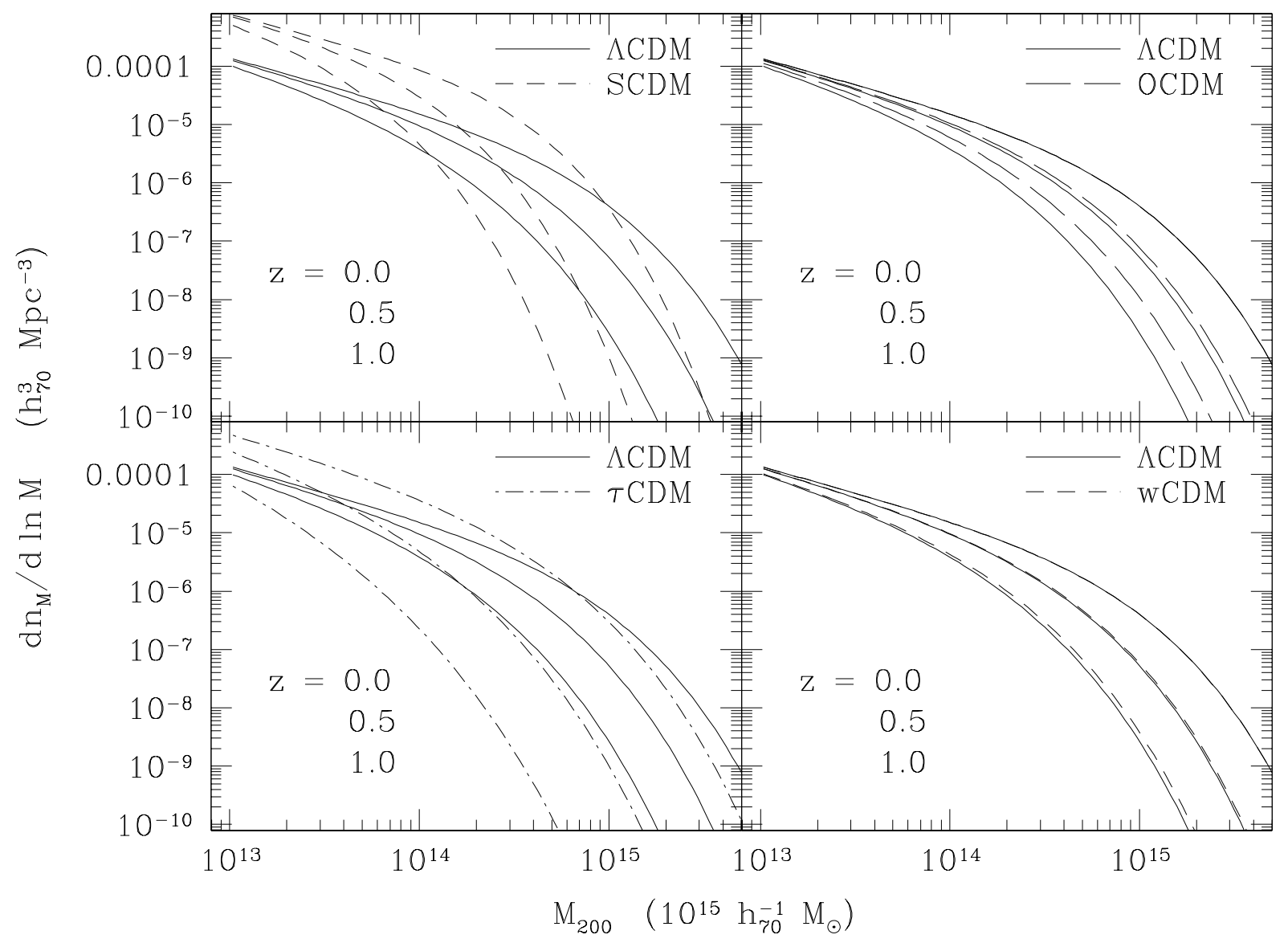

FIG. 5 Mass-function evolution in five different cosmologies. The fiducial model in all cases is the $\Lambda$ CDM model with $\Omega_{\mathrm{M}}=0.3$, $\Omega_{\Lambda}=0.7, w=-1$, and $\sigma_{8}=0.9$. The upper left panel compares cluster evolution in the $\Lambda$ CDM case with a standard colddark-matter model (SCDM) having $\Omega_{\mathrm{M}}=1.0, \Omega_{\Lambda}=0.0$, and $\sigma_{8}=0.5$. Evolution in the SCDM case is much more dramatic, and the steeper slope of the mass function strongly disagrees with observations of local clusters (e.g., Reiprich and Böhringer, 2002). Retaining $\Omega_{\mathrm{M}}=1.0$ and $\Omega_{\Lambda}=0.0$ while adjusting the power spectrum so that $\Gamma=0.21$ gives a $\tau$ CDM model (lower left) in which the slope of the low-redshift mass function is more acceptable, but the evolution is still very strong. Dispensing with dark energy while keeping the matter density low gives an OCDM model $\left(\Omega_{\mathrm{M}}=0.3, \Omega_{\Lambda}=0, \sigma_{8}=0.9\right.$; upper right) with less evolution than the $\Lambda$ CDM case because structure formation starts to ramp down earlier in time (see Figure 21). Dark energy in a $w \mathrm{CDM}$ model identical to the $\Lambda \mathrm{CDM}$ model except with $w=-0.8$ (lower right) also slows cluster evolution relative to the $\Lambda \mathrm{CDM}$ case.

of cluster mass, are also exponentially amplified by the steepness of the mass function.

\section{Cluster Bias}

Another observable feature of the cluster population, closely related to the mass function, is the tendency of galaxy clusters to cluster with one another. Fluctuations in the number density of clusters on large scales are observed to be more pronounced than the fluctuations of the underlying matter density (e.g., Bahcall et al., 2003a; Bahcall and Soneira, 1983; Collins et al., 2000; Klvpin and Kopvlov, 1983; Postman et al., 1992). In other words, the fractional deviation of $d n_{M} / d \ln \sigma^{-1}$ from its mean value within a given volume of the universe is observed to be larger than $\delta \rho / \rho$ in that same volume. The ratio $b(M)$ between the perturbation in the number density of clusters of mass $M$ and the perturbation amplitude of the matter density is known as the bias parameter, and it is taken to be independent of length scale, as long as that length scale is much larger than a cluster.

Cluster bias can be interpreted as a modulation of the collapse threshold by long-wavelength density modes (Cole and Kaiser, 1989; Kaiser, 1984; White et al., 1987). The idea here is that a long-wavelength density enhancement of amplitude $\delta \rho / \rho=\epsilon$ lowers the effective collapse threshold for smaller-scale structures to $\delta_{c}-\epsilon$, thereby inducing an offset in $d n_{M} / d \ln \sigma^{-1}$ from its mean value on mass scale $M$. This contribution adds to the perturbation $\epsilon$ in cluster number density owing to the amplitude of the large-scale mode. Dividing the sum of these two offsets by $\epsilon$ leads to an expression relating 
the bias parameter to the mass function (Mo and White, 1996; Sheth and Tormen, 1999):

$$
b(M)=1-\frac{d}{d \delta_{c}}\left[\ln \left(\frac{d n_{M}}{d \ln \sigma^{-1}}\right)\right] .
$$

Plugging in the Sheth-Tormen mass function of equation (54) produces

$$
b(M)=1+\frac{1}{\delta_{c}}\left[\frac{a_{\mathrm{s}} \delta_{c}^{2}}{\sigma^{2}}-1+\frac{2 p_{\mathrm{s}}}{1+\left(a_{\mathrm{s}} \delta_{c}^{2} / \sigma^{2}\right)^{p_{\mathrm{s}}}}\right] .
$$

$\mathrm{Hu}$ and Kravtsov (2003) show that the parameter values $a_{\mathrm{s}}=0.75$ and $p_{\mathrm{s}}=0.3$ accurately reproduce the bias of cluster-sized halos seen in large-scale numerical simulations, when cluster mass is taken to be $M_{180 \mathrm{~m}}$. Notice that small values of $\sigma(M)$ lead to large values of $b(M)$, meaning that rare, high-mass objects are much more likely to be found in regions of the universe where the surrounding matter density is higher than average.

\section{Measuring the Cluster Mass Function}

Equations (52), (54), and (55) illustrate why cosmologists are so enthusiastic about the cluster mass function. Dividing an accurate measurement of the mass function by $\Omega_{\mathrm{M}} \rho_{\mathrm{cr} 0}$ directly leads to an accurate measurement of the primordial power spectrum $\sigma(M)$ on mass scales $\sim 10^{14}-10^{15} M_{\odot}$. Furthermore, any uncertainty in $\rho_{\text {cr0 }}$ scales out of the power spectrum's normalization, because measured values of cluster number density scale as $h^{3}$, making the quantity $M_{8} \rho_{\text {cr0 }}^{-1}\left(d n_{M} / d \ln \sigma^{-1}\right)$ independent of $h$. One is left only with a degeneracy between $\sigma_{8}$ and $\Omega_{\mathrm{M}}$. Taking the logarithmic derivative of (52) with respect to $\sigma$ at constant $M$ shows that the mass function is roughly $\propto \sigma^{2}$ in the region where $\sigma \approx 1$. Hence, the measured level of that normalization in the local universe reflects the parameter combination $\sigma_{8} \Omega_{\mathrm{M}}^{\alpha}$, with $\alpha \approx 0.5$.

This degeneracy can be broken in three ways. First, one can simply measure $\Omega_{\mathrm{M}}$ or $\sigma_{8}$ in some other way. Second, one can measure the cluster mass function over a range of masses and rely on a precise measurement on the mass function's shape to break the degeneracy, assuming that the CDM power spectrum (Sec. III.A.5) is valid. Or, third, one can measure the evolution of the cluster mass function, which is highly sensitive to $\Omega_{\mathrm{M}}$. We will explore that option in more depth in Sec. III.D but first we need to examine some of the obstacles to accurate mass-function measurements.

\section{Linking Mass with Observables}

In order to measure the mass function using a large sample of clusters, we need to relate cluster mass to an easily observable quantity. Doing this properly requires a consistent definition of mass (Sec. III.B.3) and a well-calibrated relation linking that mass to some observable, but which mass definition works best? Expressions like the Jenkins mass function (equation [55) appear to be cosmologically invariant when cluster masses are defined with respect to the background mass density (e.g., $M_{180 \mathrm{~m}}$, see Sec. (II.B.4. On the other hand, the structure of a cluster, as reflected in its dark-matter velocity dispersion, seems to be cosmologically invariant when cluster masses are defined with respect to the critical mass density (e.g., $M_{200}$, see Sec.III.B.3). To paraphrase Evrard (2004), Nature appears to do accounting relative to the mean mass density and dynamics relative to the critical density.

Because simulations suggest that dynamical quantities like the galaxy velocity dispersion and the X-ray determined gas temperature should be more tightly correlated with $M_{200}$ than with $M_{180 \mathrm{~m}}$, we will take $M_{200}$ to be the primary definition of cluster mass. Methods like those outlined in Sec. III.B.3 can then be used to convert a mass function in $M_{200}$ to one in $M_{180 \mathrm{~m}}$. Alternatively, one can fit the results of large-scale simulations to determine a cosmology-dependent correction to the Jenkins mass function for use with the mass definition $M_{200}$. Evrard et al. (2002) have done that, finding that substituting $A_{\mathrm{J}}=0.27-0.07\left(1-\Omega_{\mathrm{M}}\right), B_{\mathrm{J}}=0.65+0.11\left(1-\Omega_{\mathrm{M}}\right)$, and $\epsilon_{\mathrm{J}}=3.8$ into equation (55) reproduces the $M_{200}$ mass function in simulations at $z=0$. Despite the tight relationship between $M_{200}$ and the dark-matter velocity dispersion in simulations, the link between $M_{200}$ and observable quantities is still a potentially large source of systematic error. Even if the galaxy velocity dispersion were identical to that of the dark matter, accurately measuring that dispersion within a sphere of radius $r_{200}$ requires an enormous amount of data to minimize projection effects (e.g., Rines et al., 2003).

To see how systematic errors corrupt the mass-function measurement, consider the general case for a generic observable $X$. Suppose a cluster survey determines the comoving number density distribution $d n_{M} / d \ln X$ within logarithmic bins of the observable. Converting this distribution to a mass function $d n_{M} / d \ln \sigma^{-1}$ via the chain rule requires, at minimum, knowledge of the normalization and effective power-law index $\alpha_{X} \equiv d \ln X / d \ln M$ of the $M_{200}-X$ relation over the observed range in $X$, as well as the effective power-law index $\alpha_{M} \equiv d \ln \sigma^{-1} / d \ln M$ of the mass fluctuations. Fitting a semi-analytic expression for the mass function like equation (55) to the observations for a fixed value of $\Omega_{\mathrm{M}}$ then determines $\sigma_{\text {fit }}=\sigma\left(M_{\text {fit }}\right)$ on a particular mass scale $M_{\text {fit }}$, and consequently determines $\sigma_{8} \approx\left(M_{\text {fit }} / M_{8}\right)^{\alpha_{M}} \sigma_{\text {fit }}\left(M_{\text {fit }}\right)$.

Any systematic offset $\Delta M / M$ in the normalization of the $M_{200}-X$ relation produces a corresponding offset in the measured power-spectrum normalization:

$$
\begin{aligned}
\frac{\Delta \sigma_{8}}{\sigma_{8}} & =\left(\alpha_{M}+\frac{d \ln \sigma_{\mathrm{fit}}}{d \ln M_{\mathrm{fit}}}\right) \frac{\Delta M}{M} \\
& =\alpha_{X}\left[\alpha_{M}+\frac{1}{\epsilon_{\mathrm{J}}\left(\ln \sigma^{-1}+B_{\mathrm{J}}\right)^{\epsilon_{\mathrm{J}}-1}}\right] \frac{\Delta X}{X}
\end{aligned}
$$

(e.g., Evrard et al., 2002; Seliak, 2002; Voit, 2000). The second line of this equation assumes that $\sigma_{\text {fit }}$ has been 
determined using the Jenkins mass function of equation (55). On the mass scale of rich clusters $\left(\gtrsim 10^{14.5} M_{\odot}\right)$, Evrard et al. (2002) find that the factor in parentheses is $\approx 0.4$. implying that a systematic $25 \%$ error in mass would lead to a $10 \%$ error in the measurement of $\sigma_{8}$. Below this mass scale the factor in parentheses increases, leading to an even larger error in the power-spectrum normalization for a given mass offset (Huterer and White, 2002).

Dispersion in the value of the mass-tracing observable for clusters of fixed mass is another important source of uncertainty that must be dealt with carefully because of the exponential slope of the mass function (e.g., Pierpaoli et al., 2003). Significant scatter boosts the normalization of $d n_{M} / d \ln X$ over the expectation for the noscatter case, as the overall number of lower-mass clusters scattering to higher values of $X$ far exceeds the number of higher-mass clusters scattering in the opposite direction. Underestimating this scatter leads to an overestimate of $\sigma_{8}$ that can be particularly severe if the scatter has a long non-gaussian tail to large values of $X$. Situations in which such a tail could arise include merger shocks that substantially boost the temperature and luminosity in a significant subset of X-ray selected clusters (e.g., Randall et al., 2002; Ricker and Sarazin, 2001) and superpositions of galaxies that boost the apparent richness and velocity dispersion in an optically-selected sample (van Haarlem et al., 1997).

Surveys that probe deep into the universe for clusters must also cope with redshift evolution in the massobservable relation. That is, if $M_{200} \propto X^{\alpha_{X}}(1+z)^{b_{X}}$, then one needs to know the value of $b_{X}$. This source of uncertainty affects both the mapping of $X$ onto mass for individual clusters and the number density one infers for clusters of a given mass from a survey based on the observable $X$.

A sufficiently large cluster survey can circumvent many of these systematic problems through self-calibration $(\mathrm{Hu}$, 2003; Levine et al., 2002; Majumdar and Mohr, 2003, 2004). This procedure treats all parameters describing the systematic uncertainties in such things as the scatter, normalization, and evolution of the massobservable relation as free parameters in the overall cosmological model. Fitting a large number of clusters spanning a wide range in redshift to this overall model, one can then determine not only the global cosmological parameters but also the most likely values of the free parameters in the mass-observable relation. However, treating the systematic uncertainties in this way has a cost. Each free parameter added weakens the statistical constraints on the cosmological measurements (Maiumdar and Mohr, 2003).

\section{Mass-Temperature Relation}

Among the observables that trace cluster mass, X-ray temperature has received considerable recent attention because it is closely related to the depth of a cluster's potential well and can be readily observed to $z \sim 1$ with current X-ray telescopes (Sec. II.B.2). Henry and Arnaud (1991) pioneered the technique of measuring the cluster mass function with X-ray temperatures, using cluster temperatures determined at $z \approx 0$ with the Einstein, Exosat, and $H E A O / O S O$ satellites. Cluster temperatures measured with the $A S C A$ satellite improved the precision of this measurement (Ikebe et al., 2002), and temperatures measured with the Chandra and XMM-Newton telescopes should improve that precision even more. Because the data are now of such high quality, systematic uncertainty in the link between mass and temperature is the main factor limiting this technique.

Mass and temperature ought to be simply related for a cluster in hydrostatic equilibrium. The gas temperature of a singular isothermal sphere with mass $M_{200}$ inside radius $r_{200}$ is

$$
\begin{aligned}
k_{\mathrm{B}} T_{200} & =\frac{G M_{200} \mu m_{p}}{2 r_{200}}=\frac{\mu m_{p}}{2}\left[10 G M_{200} H(z)\right]^{2 / 3} \\
& =(8.2 \mathrm{keV})\left(\frac{M_{200}}{10^{15} h^{-1} M_{\odot}}\right)^{2 / 3}\left[\frac{H(z)}{H_{0}}\right]^{2 / 3}
\end{aligned}
$$

Realistic departures from hydrostatic equilibrium can be assessed with simulations of structure formation that include hydrodynamics, but they do not have a large effect on the mass-temperature relation. These models indeed find that gas temperature scales with $M_{200}^{2 / 3}$, so that $T \approx T_{200}$, with a scatter of only $10-15 \%$ (e.g., Evrard et al., 1996; Frenk et al., 1999). However, to calibrate the mass-temperature relation more precisely, we need a more specific definition of temperature.

Clusters are not perfectly isothermal, so any single number specifying a cluster's gas temperature is some sort of weighted mean. The luminosity-weighted mean temperature $T_{\text {lum }}$ obtained by weighting each gas parcel's temperature by $\propto \rho_{g}^{2}$ is a popular choice for comparing theory with observations because each temperature component contributes in proportion to its photon flux in the cluster's overall spectrum (Sec. II.B.2). The spectralfit temperature $T_{\mathrm{sp}}$ has not yet received much attention in theoretical work because it depends somewhat on the procedure used to fit the spectrum, but Mazzotta et al. (2004) have recently developed a temperature weighting scheme for theoretical models that appears to track $T_{\mathrm{sp}}$ quite closely.

A bewildering variety of parameters has been used in the literature to express the mass-temperature relation's normalization. Here we will express the normalization of the $M_{200}-T_{\text {lum }}$ relation in terms of $T_{\text {lum }} / T_{200}$. This choice has two advantages: It does not link the normalization to any particular mass or temperature scale, and $T_{\text {lum }} / T_{200}=1$ for both a singular isothermal sphere and an isothermal beta model with $\beta=2 / 3$.

Uncertainty in this normalization factor is currently the single most important issue afflicting cluster massfunction measurements with X-ray observations. Table 
TABLE I Normalization of the Mass-Temperature Relation

\begin{tabular}{|c|c|}
\hline Models without Radiative Cooling & $T_{\text {lum }} / T_{200}{ }^{a}$ \\
\hline Navarro et al. (1995) & 0.99 \\
\hline Evrard et al. $(1996)^{b}$ & 0.91 \\
\hline Brvan and Norman (1998) & 0.80 \\
\hline Thomas et al. (2001) & 0.98 \\
\hline Muanwong et al. $(2002)^{c}$ & 0.57 \\
\hline Muanwong et al. $(2002)^{d}$ & 0.90 \\
\hline Observations & $\overline{T_{\text {lum }} / T_{200}{ }^{a}}$ \\
\hline$\overline{\text { Horner } \text { et al. }(1999)^{e}}$ & $(1.08 \pm 0.04) T_{6}^{-}$ \\
\hline Horner et al. $(1999)^{f}$ & $(1.40 \pm 0.16) T_{6}^{-0.02}$ \\
\hline Nevalainen et al. $(2000)^{g}$ & $(1.20 \pm 0.12) T_{6}^{-0.20}$ \\
\hline Finoguenov et al. $(2001 \mathrm{~b})^{b, h}$ & $(1.18 \pm 0.10) T_{6}^{-0.33}$ \\
\hline Finoguenov et al. $(2001 \mathrm{~b})^{b, i}$ & $(1.26 \pm 0.11) T_{6}^{-0.19}$ \\
\hline Finoguenov et al. $(2001 \mathrm{~b})^{b, j}$ & $(1.33 \pm 0.18) T_{6}^{-0.02}$ \\
\hline Models with Radiative Cooling & $\overline{T_{\text {lum }} / T_{200}{ }^{a}}$ \\
\hline Muanwong et al. (2002) & $0.79 T_{6}^{-0.31}$ \\
\hline Muanwong et al. $(2002)^{k}$ & $0.88 T_{6}^{-0.09}$ \\
\hline Borgani et al. $(2003)^{b}$ & $(1.03 \pm 0.03) T_{6}^{-0.06}$ \\
\hline Borgani et al. $(2003)^{b, l}$ & $(1.24 \pm 0.03) T_{6}^{-0.06}$ \\
\hline
\end{tabular}

${ }^{a} T_{6} \equiv k_{\mathrm{B}} T_{\mathrm{lum}} / 6 \mathrm{keV}$

${ }^{b}$ Conversion from $M_{500}$ assumes $M_{200}=1.4 M_{500}$.

${ }^{c} T_{\text {lum }}$ computation includes gas with cooling time $<6 \mathrm{Gyr}$

${ }^{d} T_{\text {lum }}$ computation excludes gas with cooling time $<6 \mathrm{Gyr}$

${ }^{e}$ Masses estimated using isothermal beta model

$f_{\text {Masses estimated using polytropic beta model }}$

${ }^{g}$ Conversion from $M_{1000}$ assumes $M_{200}=2.0 M_{1000}$.

${ }^{h}$ Full sample, masses from isothermal beta model

${ }^{i}$ Full sample, masses from polytropic beta model

${ }^{j}$ Subset with $k_{\mathrm{B}} T_{\text {lum }}$, polytropic beta model

${ }^{k} T_{\text {lum }}$ computed with cooling cores removed

${ }^{l}$ Masses inferred from polytropic beta-model fits.

provides some recent observational and theoretical calibrations of this relation, in three different groups. The first group gives calibrations from hydrodynamical simulations that do not account for galaxy formation, which generally fall into the range $T_{\text {lum }} / T_{200}=0.8-1.0$. In some cases, an $M_{500}-T_{\text {lum }}$ relation has been converted to $M_{200}$ assuming $M_{200}=1.4 M_{500}$, appropriate for halo concentration $c=5$. The second group gives calibrations inferred from observations, which fall into the range $T_{\operatorname{lum}} / T_{200}=1.1-1.4$. In other words, clusters of a given temperature seem to be $30 \%$ to $60 \%$ less massive than one would expect from the simulations. Apparently galaxy formation changes the normalization of the $M_{200}-T_{\text {lum }}$ relation, for reasons discussed in detail in Sec. IV although some of this discrepancy may also stem from systematic offsets in the observational interpretation. The third group of normalization factors, which tend to lie in between the first two groups, come from simulations that attempt to account for the effects of galaxy formation. Given this uncertainty in the mass-temperature normalization, the systematic uncertainty in $\sigma_{8}$ values derived from cluster temperatures is about $25 \%$, because $\sigma_{8} \propto\left(T_{1 u m} / T_{200}\right)^{3 / 5}$ according to equation (59) for rich clusters (Evrard et al., 2002).

Efforts are underway to reconcile the observed nor- malization of the $M_{200}-T_{\text {lum }}$ relation with theoretical expectations. Some of the discrepancy probably arises from systematic errors in the masses derived from Xray observations. Hydrostatic equilibrium is usually assumed, but the turbulent velocities in simulated clusters can sometimes be $\sim 20-30 \%$ of the sound speed, in which case the hydrostatic assumption would lead to masses underestimated by 10-15\% (Rasia et al., 2003; Ricker and Sarazin, 2001). In addition, the beta model formalism often used to derive cluster mass may have systematic problems. Applying this model to simulated clusters tends to underestimate their masses (Borgani et al., 2003; Muanwong et al., 2002, see the last line of Table [), and recent XMM-Newton observations suggest that the correction for temperature gradients may be excessive (Mushotzkv, 2004; Pratt and Arnaud, 2002, 2003).

Alternative mass measurements would be very helpful in solving these problems. Calibration of the masstemperature relation with lensing observations has met with mixed success. Weak-lensing measurements of massive, relaxed clusters agree with the masses derived from $\mathrm{X}$-ray data under the assumption of hydrostatic equilibrium (Allen et al., 2001). However, that agreement seems to be poorer for less relaxed clusters (Smith et al., 2003). Measurements of cluster mass from the galaxy velocity field in and around a few very well observed clusters also tend to support the X-ray derived masses Rines et al., 2003). Because the calibration may depend systematically on how clusters are selected, self-calibration of a large cluster survey may ultimately be the best way of calibrating the $M_{200}-T_{\text {lum }}$ relation. A thorough understanding of how galaxy formation affects that relation would help reduce the number of free parameters that need to be calibrated, thereby reducing the statistical uncertainties achievable with self-calibration.

\section{Mass-Luminosity Relation}

X-ray luminosity also correlates well with cluster mass and is easier to measure than X-ray temperature, allowing for mass-function measurements using much larger cluster samples. However, the correlation between mass and luminosity is not as tight as that between mass and temperature, having a scatter $\sim 50 \%$ (Reiprich and Böhringer, 2002). Additionally, the normalization and slope of the relation depend heavily on the physics of galaxy formation (Sec.IV.Cl). Because our understanding of the connection between galaxy formation and a cluster's X-ray luminosity is not yet mature enough to calibrate the mass-luminosity relation with simulations, we need to rely solely on observational calibrations.

One common way to calibrate the mass-luminosity relation is to combine the mass-temperature relation with the observed luminosity-temperature relation (e.g., Borgani et al., 1999). On cluster scales, the relation between the total (bolometric) X-ray luminosity and 
TABLE II Luminosity-Temperature Relation at $z \approx 0$

\begin{tabular}{lcc}
\hline \hline Source & $L_{6}{ }^{a}$ & $\alpha_{L T}$ \\
\hline Edge and Stewart (1991) & $6.3 \pm 1.3$ & $2.62 \pm 0.10$ \\
David et al. (1993) & $5.6 \pm 0.9$ & $3.37 \pm 0.05$ \\
Markevitch $(1998)^{b}$ & $6.4 \pm 0.6$ & $2.64 \pm 0.27$ \\
Allen and Fabian (1998) $^{c}$ & $5.7 \pm 3.4$ & $2.92 \pm 0.45$ \\
Allen and Fabian $(1998)^{d}$ & $14.6 \pm 7.3$ & $3.08 \pm 0.58$ \\
Arnaud and Evrard (1999) $^{e}$ & $5.9 \pm 0.4$ & $2.88 \pm 0.15$ \\
Xue and Wu (2000) & $7.6 \pm 1.2$ & $2.79 \pm 0.08$ \\
Novicki et al. (2002) & $6.0 \pm 4.2$ & $2.82 \pm 0.43$ \\
Ettori et al. (2002) & $7.3 \pm 1.8$ & $2.54 \pm 0.42$ \\
\hline \hline
\end{tabular}

${ }^{a}$ Bolometric X-ray luminosity is $L_{\mathrm{X}}=L_{6}\left(T_{\mathrm{lum}} / 6 \mathrm{keV}\right)^{\alpha_{L T}}$ with $L_{6}$ in units of $10^{44} h_{70}^{-2} \mathrm{erg} \mathrm{s}^{-1}$.

${ }^{b}$ Cores of clusters excised to avoid cool cores.

${ }^{c}$ Clusters without cool cores.

${ }^{d}$ Clusters with cool cores.

${ }^{e}$ Sample avoids clusters with cool cores.

$T_{\text {lum }}$ is approximately a power law. Normalizing the relation at $6 \mathrm{keV}$, in the heart of the temperature range for rich clusters, leads to the expression $L_{\mathrm{X}}=$ $L_{6}\left(T_{\text {lum }} / 6 \mathrm{keV}\right)^{\alpha_{L T}}$, and Table [1] gives some representative values of $L_{6}$ and $\alpha_{L T}$. Excising the central regions of clusters, out to about $100 \mathrm{kpc}$ in radius, reduces the scatter in the relation because cooling and nongravitational heating processes affect the temperature and luminosity of these regions differently from cluster to cluster (Allen and Fabian, 1998; Fabian et al., 1994; Markevitch, 1998; Voit et al., 2002).

The power-law index of the $L_{\mathrm{X}}-T_{\text {lum }}$ relation clearly indicates that galaxy formation has affected the $L_{\mathrm{X}}-T_{\text {lum }}$ relation. If the density distribution of intracluster gas within $r_{200}$ were self-similar, independent of cluster mass, then one would expect bremsstrahlung emission to give $L_{\mathrm{X}} \propto \rho_{g} M_{200} T_{\text {lum }}^{1 / 2} \propto T_{\text {lum }}^{2}$ Kaiser, 1986). The steepness of the observed power-law index indicates that nongravitational processes have raised the entropy of the intracluster gas, making it harder to compress, particularly in the shallower potential wells of cool clusters. This excess entropy therefore lowers the luminosities of all clusters by lowering the mean gas density and steepens the $L_{\mathrm{X}}-T_{\text {lum }}$ relation because the impact of excess entropy decreases as cluster temperature rises (Evrard and Henrv, 1991; Kaiser, 1991, Sec. IV.C.

Calibrating the mass-luminosity relation by coupling the mass-temperature relation with the observed luminosity-temperature relation leads, not surprisingly, to values of $\sigma_{8}$ that are similar to those derived from the mass-temperature relation alone and are subject to the same systematic uncertainties that plague the masstemperature calibration. There is, however, a route to the mass-luminosity calibration that circumvents the middle step involving the mass-temperature relation.

The mass-luminosity relation can be calibrated more directly with high-quality X-ray imaging and temperature data on a complete sample of clusters. Reiprich and Böhringer (2002) have done this with
ROSAT imaging data and $A S C A$ temperatures, finding

$$
L_{\mathrm{X}}=10^{45.0 \pm 0.3} h_{70}^{-2} \operatorname{erg~s}^{-1}\left(\frac{M_{200}}{10^{15} h_{70}^{-1} M_{\odot}}\right)^{1.8} .
$$

Their mass calibration assumes that the cluster gas is in hydrostatic equilibrium and obeys an isothermal beta model. The masses they derive are therefore higher than those one would find after correcting for a possible negative temperature gradient at large radii but do not account for any turbulent pressure support. With this mass-luminosity relation, they find a cluster mass function whose normalization corresponds to $\sigma_{8}=0.68\left(\Omega_{\mathrm{M}} / 0.3\right)^{-0.38}$.

Furthermore, because their observed cluster sample extends over two decades in mass, Reiprich and Böhringer (2002) attempted to break the $\sigma_{8}-\Omega_{\mathrm{M}}$ degeneracy by fitting the mass-function's shape with a CDM power spectrum, finding a best fit of $\Omega_{\mathrm{M}}=0.12_{-0.04}^{+0.06}$ and $\sigma_{8}=$ $0.96_{-0.12}^{+0.15}$, with $\Omega_{\mathrm{M}}<0.31$ at the $3 \sigma$ level. The unusually low best-fit value of $\Omega_{\mathrm{M}}$ arises because their derived mass function is shallower than that expected for $\Omega_{\mathrm{M}}=0.3$. However, Pierpaoli et al. (2003) have applied that same $L_{X}-M_{200}$ relation to the larger REFLEX cluster sample (Böhringer et al., 2002), finding $\sigma_{8}=0.86_{-0.16}^{+0.12}$ and $\Omega_{\mathrm{M}}=0.23_{-0.06}^{+0.10}$. Results similar to these latter values are also found from the mass-luminosity relation when cluster evolution is used to break the $\sigma_{8}-\Omega_{\mathrm{M}}$ degeneracy (Sec. III.D) .

\section{Mass-Richness Relation}

Optical telescopes have gathered much larger cluster samples than have X-ray telescopes, but deriving a mass function from these samples is not so straightforward. Projection effects complicate both the measurement of cluster mass and the computation of the sample volume associated with a given mass. Clusters in optical surveys are selected on the basis of richness, which depends on the number of galaxies observed within a certain projected radius from the center of the cluster (Sec. II.A.1). Thus, even if optical luminosity traces mass exactly, galaxy concentrations lying outside $r_{200}$ but projected along the same line of sight can boost the apparent mass, introducing non-gaussian uncertainties in the mass-richness relation. Likewise, the effective volume associated with a given cluster mass in an richness-selected survey is harder to quantify than in a survey with a definite $\mathrm{X}$ ray flux cutoff. Nevertheless, when richness is rigorously defined, it correlates well with a cluster's X-ray properties Yee and Ellingson, 2003). However, the scatter between optical richness and X-ray luminosity is still large compared with the accuracy to which one would like to derive cosmological parameters (Donahue et al., 2001, 2002; Gilbank et al., 2003; Kochanek et al., 2003).

Measuring cluster masses purely on the basis of galaxycount richness necessitates a different approach to defin- 
ing a cluster's radius and therefore its mass. Because traditional measures of richness depend on the radius within which galaxies are counted, they are defined with respect to a fixed physical radius, independent of mass, at each redshift. For this reason, observations of cluster richness are sometimes compared with simulations on the basis of cluster masses measured within a constant physical or comoving radius (Bode et al., 2001). Deriving a massrichness relation from simulations of galaxy formation also involves an observational calibration of the cluster mass-to-light ratio, which according to equation (59) introduces a systematic uncertainty in $\sigma_{8}$ that is $\sim 40 \%$ of the uncertainty in the mass-to-light conversion.

Making such a comparison with the early clusters from the Sloan Digital Sky Survey, Bahcall et al. (2003b) find a mass-function normalization implying $\sigma_{8}=0.69 \pm$ $0.07\left(\Omega_{\mathrm{M}} / 0.3\right)^{-0.6}$. Adding mass-function shape information to break the degeneracy leads to $\Omega_{\mathrm{M}}=0.19_{-0.07}^{+0.08}$ and $\sigma_{8}=0.9_{-0.2}^{+0.3}$, in reasonably good agreement with the $\mathrm{X}$-ray derived values. Unfortunately, because there is as yet no simple parametric form, analogous to the Jenkins mass function, giving the mass function defined with respect to a fixed radius, it is not clear how to self-calibrate the associated mass-richness relation to high accuracy with a large survey.

\section{Velocity Dispersion and Mass}

Velocity dispersion is the optical analog to X-ray temperature. Thus, one would expect a mass function defined on the basis of velocity dispersion to coincide with those defined with respect to cluster temperature. Measurements of rich clusters indicate that $\sigma_{1 \mathrm{D}}^{2}=(1.0 \pm$ $0.1) k_{\mathrm{B}} T_{\text {lum }} / \mu m_{p}$ (e.g., Xue and Wu, 2000, Sec. II.B.2), which reassuringly suggests that both quantities accurately trace mass. On the other hand, Evrard et al. (2002) have pointed out that combining this relation with the observational calibration of the $M_{200}-T_{\text {lum }}$ relation ( $T_{\text {lum }} \approx 1.2 T_{200}$ ) leads to a puzzle. While it might be possible for non-gravitational effects associated with galaxy formation to boost $T_{\text {lum }}$, it is more difficult to imagine why non-gravitational effects would boost the galaxy velocity dispersion by a similar factor.

Mass functions derived from velocity dispersion measurements also suggest that the masses derived from those measurements are larger than those derived from X-ray data. Using the virial theorem with a pressure correction term (Sec. II.A.2), Girardi et al. (1998) derive a cluster mass function from velocity dispersions whose normalization indicates $\sigma_{8}=(1.01 \pm 0.07)\left(\Omega_{\mathrm{M}} / 0.3\right)^{-0.43}$, implying an overall number density at a given cluster mass about two times larger than the X-ray measurements. A discrepancy in $\sigma_{8}$ as large as $30 \%$ could arise if the optically determined masses were over $50 \%$ larger, but the actual mass discrepancies appear not to be quite so large. Reiprich and Böhringer (2002) find that in the 42 clusters they have in common with Girardi et al.
(1998) the virial masses are $25 \%$ larger, on average, than the X-ray masses. Another factor that could contribute to this discrepancy is scatter in the $M_{200}-\sigma_{1 \mathrm{D}}$ relation. An underestimate of the scatter would drive up the inferred mass-function amplitude, raising the best-fitting value of $\sigma_{8}$.

Part of the discrepancy between the optical and X-ray determined masses may stem from how velocity dispersions are observed. Because $\sigma_{1 \mathrm{D}}$ declines with projected radius, its observed value will depend on the cutoff radius. Also, any foreground or background interlopers projected onto the cluster can contaminate the velocitydispersion measurement. Ideally, one would like to cut off the measurement at a spherical boundary with radius $r_{200}$, inside of which the relation between dark-matter velocity dispersion and mass is well-calibrated, but the large number of galaxy velocities needed to accurately measure the mass profile near the virial radius make this approach impractical for large cluster samples. In a small sample of eight rich clusters, Rines et al. (2003) have used an average of almost 200 galaxy velocities per cluster, extending to well beyond $r_{200}$, to measure the mass $M_{200}$ within $r_{200}$. The masses they find are consistent with both the X-ray determined masses and with the virial theorem including a surface-pressure correction.

\section{Weak Lensing and Mass}

Weak lensing is a very promising method for measuring cluster masses that is independent of a cluster's baryon content, dynamical state, and mass-to-light ratio. The main systematic problem in weak-lensing mass measurements comes from the lensing done by excess mass outside the virial radius but along the line of sight through the cluster (Hoekstra, 2001; Metzler et al., 2001, 1999). So far, weak-lensing's main contribution to cluster studies has been to assist in the calibration of other mass estimators (e.g., Allen et al., 2001).

Techniques for compiling cluster samples selected on the basis of weak lensing are still in their infancy. Only a few clusters with confirmed spectroscopic redshifts have been detected in weak lensing surveys (e.g. Dahle et al., 2003; Schirmer et al., 2003; Wittman et al., 2003, 2001). However, deep optical surveys covering wide patches of the sky should turn up many more such clusters in the coming decade. In the meantime, smaller weaklensing surveys sensitive to large-scale structure are complementing the cluster work because they provide values of $\sigma_{8}$ that are independent of the cluster measurements. Numbers currently in the literature span approximately the same range as those derived from clusters, going from $\sigma_{8}=(0.72 \pm 0.08)\left(\Omega_{\mathrm{M}} / 0.3\right)^{-0.57}$ Jarvis et al. , $2003)$ on the low end to $\sigma_{8}=(0.97 \pm 0.14)\left(\Omega_{\mathrm{M}} / 0.3\right)^{-0.44}$ (Bacon et al., 2003) on the high end. 


\section{Baryons and Mass}

Yet another technique for measuring the cluster mass function relies on the constancy of the ratio of baryons to dark matter in massive clusters. X-ray observations from Chandra indicate that the ratio of hot baryonic gas to total gravitating matter within a given radius asymptotically approaches $(0.113 \pm 0.005) h_{70}^{-3 / 2}$ in relaxed, highmass clusters (Allen et al., 2002). Correcting for the baryons in stars, whose mass is approximately $0.16 h_{70}^{1 / 2}$ times that of the hot gas (Sec. IV.D raises the overall ratio of baryons to dark matter in clusters to $f_{b}=0.13$ for $h_{70}=1.0$.

This ratio is itself one of the best tools for measuring $\Omega_{\mathrm{M}}$ (Allen et al., 2002; David et al., 1995; Evrard, 1997; White et al., 1993). No known hydrodynamic process can drive a large proportion of a rich cluster's baryons out of the cluster's deep potential well. Thus, the ratio of baryons to dark matter in a cluster is expected to be similar to the global ratio in the universe. Dividing the mean baryon density $\Omega_{\mathrm{b}}=0.045 h_{70}^{-2}$ consistent with both the abundances of light elements (Burles et al., 2001) and microwave background fluctuations (Spergel et al., 2003) by the baryon fraction $f_{b}$ implies $\Omega_{\mathrm{M}} \approx 0.3$. Allen et al. (2002) find $\Omega_{\mathrm{M}}=0.30_{-0.03}^{+0.04}$ after marginalizing over the uncertainties in $\Omega_{\mathrm{b}}$ and Hubble's constant.

One can also use ratio of baryons to dark matter to constrain dark energy (Pen, 1997; Sasaki, 1996). Measurements of this ratio in clusters depend on the relationship between transverse size and redshift, which depends on both $\Omega_{\mathrm{M}}$ and $\Omega_{\Lambda}$ (Sec. III.A.2). If the actual ratio remains constant with redshift, then the measured ratios will be independent of redshift only if the correct values of $\Omega_{\mathrm{M}}$ and $\Omega_{\Lambda}$ are used in the measurement. Allen et al. (2004) have recently shown that the measured baryon to dark matter ratio in a sample of 26 clusters ranging up to $z \approx 0.9$ is consistent with the low-redshift ratio for $\Omega_{\Lambda}=0.94_{-0.23}^{+0.21}$ (see also Allen et al., 2002; Ettori et al., 2003). However, the degree to which the actual ratio is redshift-independent is not yet known.

If the ratio of baryons to dark matter were completely independent of cluster mass and radius, then measurements of the baryon mass inside a radius containing a mean baryon density of $200 f_{b} \rho_{\text {cr }}$ would directly give $M_{200}$. The cluster mass function could then be determined by measuring the baryon masses within a given scale radius (Vikhlinin et al., 2003; Voevodkin and Vikhlinin, 2004). In fact, the baryon fraction is not quite constant in clusters, probably owing to the same galaxy-formation effects that shift the $M-T$ and $L-T$ relations (Sec. IV.C). For example, Mohr et al. (1999) find that the ratio of gas mass to dark matter is $\propto T_{\text {lum }}^{0.36 \pm 0.22}$ in clusters cooler than about $6 \mathrm{keV}$ and is statistically inconsistent with a constant value at the $99 \%$ level. Other studies concur that the proportion of hot gas in low-mass clusters is smaller than that in high-mass clusters (Neumann and Arnaud, 2001; Sanderson et al., 2003).
After correcting for this effect, Voevodkin and Vikhlinin (2004) infer a cluster mass function from the baryon mass function signifying $\sigma_{8}=0.72 \pm 0.04$ for the assumed cosmology $(\Omega=0.3$, $\Omega_{\Lambda}=0.7$, and $\left.h=0.71\right)$. Furthermore, the shape parameter $\Gamma=0.13 \pm 0.07$ of the mass function is consistent with the CDM power spectrum given the assumed values of $\Omega_{\mathrm{M}}$ and $h$. Notice that this value of $\sigma_{8}$ agrees with those derived from the observationally calibrated $M_{200}-T_{\text {lum }}$ and $M_{200}-L_{\mathrm{X}}$ relations, even though it does not explicitly rely on those calibrations.

\section{Evolution of the Mass Function}

Measurements of evolution in the cluster mass function can considerably tighten all these constraints on cosmological parameters. What we actually observe, of course, is the dependence on redshift of the observables that trace the cluster mass function. For a given cluster sample we can measure the number of clusters $d N$ within a given solid angle $d \Omega$ and redshift interval $[z, z+d z]$ that fall into the range $[X, X+d X]$ of the observable $X$. With full knowledge of the mass-observable relation $M(X, z)$ and its scatter as a function of redshift, we could then derive the redshift distribution

$$
\frac{d^{3} N}{d M d \Omega d z}(M, z)=\frac{d n_{M}}{d M}(M, z) \cdot \frac{d^{2} V_{\mathrm{co}}}{d z d \Omega}(z)
$$

for clusters of mass $M$ directly from the observations. This distribution of clusters with redshift would then provide strong constraints on cosmological models through both the mass-function evolution factor $d n_{M} / d M$ and the comoving volume factor $d^{2} V_{\text {co }} / d \Omega d z$ from equation (21).

As the reader probably suspects by now, our ability to constrain cosmological parameters through the redshift distribution of clusters is currently limited by our understanding of evolution in the mass-observable relations. However, this problem is not as severe as one might expect because the evolution in the mass function itself is so dramatic, especially for $\Omega_{\mathrm{M}} \approx 1$. This part of the review discusses what we have learned about structure formation and cosmological parameters by observing cluster evolution. It begins with a description of how mass-function evolution depends on cosmological parameters and then considers the complications arising from evolution of the observables themselves. It concludes with a summary of current constraints on $\Omega_{\mathrm{M}}$ from cluster evolution and a look at the prospects for measuring $\Omega_{\Lambda}$ and $w$ with large cluster surveys.

\section{Dependence on Cosmology}

Evolution of the mass function is highly sensitive to cosmology because the matter density controls the rate at which structure grows. When the mass function can be expressed in terms of formulae like (52), 
(54), or (55), its evolution is controlled entirely by the growth function $D(z)$, which is a well defined function of $\Omega_{\mathrm{M}}, \Omega_{\Lambda}$, and $w$ (Sec. III.B.4). Smallamplitude density perturbations grow as $D(z)=(1+$ $z)^{-1}$ when $\Omega_{\mathrm{M}}(z) \approx 1$, but perturbation growth stalls when $\Omega_{\mathrm{M}}(z) \ll 1$. This effect manifests itself most strongly in high-mass clusters because they are the latest objects to form in a hierarchical cosmology with a CDM-like power spectrum (Eke et al., 1996; Evrard, 1989; Oukbir and Blanchard, 1992; Peebles et al., 1989; Viana and Liddle, 1996). The exponential dependence of the mass function on $\sigma(M, z)=D(z) \sigma(M, 0)$ makes the effect quite dramatic for objects sufficiently massive that $\sigma(M, 0)<1$

Dependence of the mass function on $\Omega_{\Lambda}$ and $w$ is a little more subtle. These parameters affect mass-function evolution by altering the redshift at which $\Omega_{\mathrm{M}}(z)$ departs significantly from unity for a given value of $\Omega_{\mathrm{M}}$ at $z=0$ (Haiman et al., 2001). The time at which dark energy begins to dominate the dynamics of the universe is later for both larger values of $\Omega_{\Lambda}$ and smaller (more negative) values of $w$ (see Figure 21), leading to greater evolution of the mass function between $z \sim 1$ and the present (Battve and Weller, 2003; Wang and Steinhardt, 1998; Weller et al., 2002).

Measurements of how the mass function changes with redshift can provide additional information about $\Omega_{\Lambda}$ and $w$ through the expansion rate of the universe. If the mass function of clusters is precisely known, then number counts of clusters exceeding a given mass in each redshift interval $d z$ reveal the volume associated with that redshift interval and can be used to determine the dynamics of the universe's expansion. The number of clusters with mass $>M$ on the celestial sphere in the redshift interval $d z$ is given by

$$
\frac{d N}{d z}(M)=\frac{4 \pi r_{\kappa}^{2}(z) c}{H(z)} n_{M}(M, z)
$$

Figure 6] shows this number-redshift distribution for several different cosmological models. Notice that the statistical power of cluster surveys is ultimately limited by the total number of massive clusters in the observable universe, which is of order $10^{5}$.

\section{Evolution of the Observables}

All of the mass-observable relations discussed in Sec. III.C evolve with redshift, partly because the definition of $M_{200}$ is pinned to the critical density and partly because of galaxy-formation physics. Clusters of a given mass are hotter earlier in time because their matter density is larger; both $T_{200}$ and the square of the dark-matter velocity dispersion for a fixed value of $M_{200}$ vary with redshift as $H^{2 / 3}(z)$ (Sec. III.C.2). One therefore expects $T_{\text {lum }}$ and the square of the galaxy velocity dispersion to depend on redshift in the same way, but it is possible that the physics of galaxy formation adds additional redshift

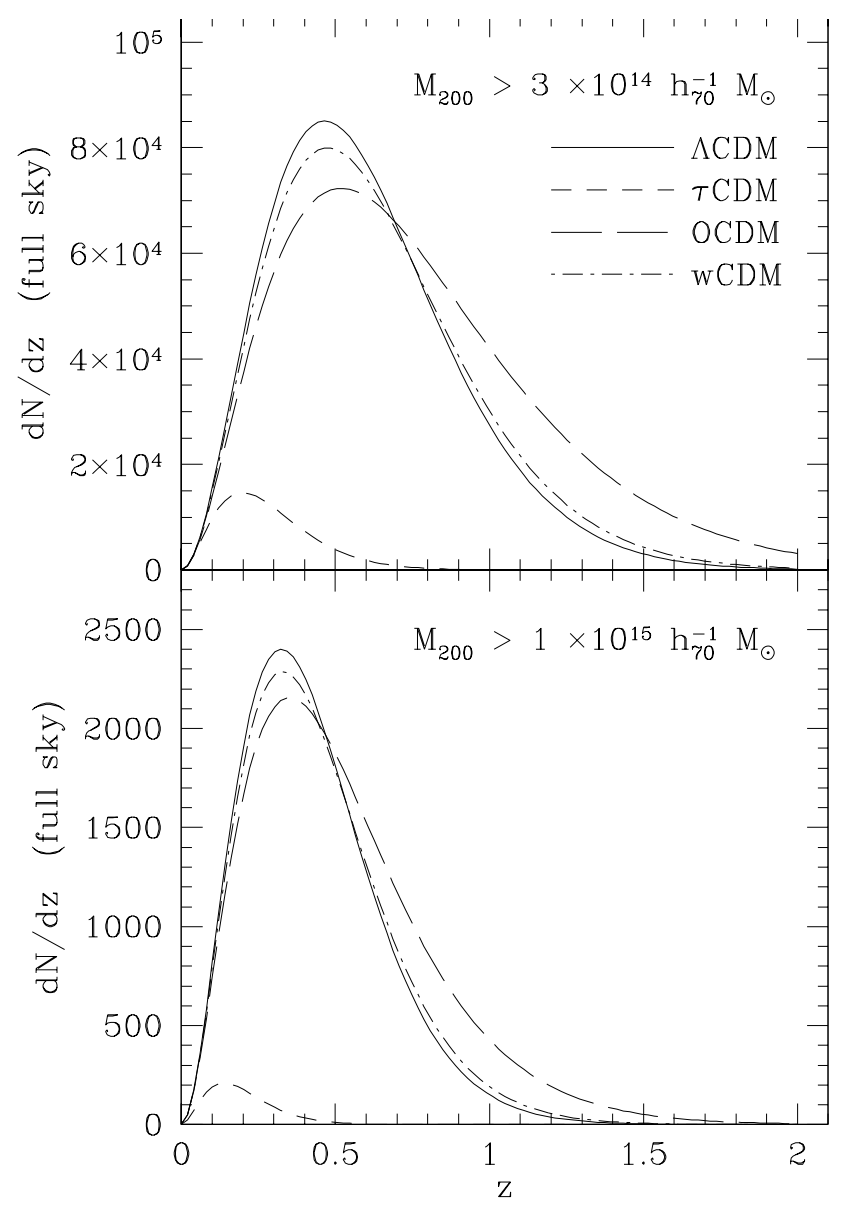

FIG. 6 Predicted number of clusters on the sky as a function of redshift in different cosmologies. The upper panel shows the number of clusters per unit redshift with $M_{200}>$ $3 \times 10^{14} h_{70} M_{\odot}$ over the entire sky. Notice that there are a few tens of thousands of such clusters on the sky in models with $\Omega_{\mathrm{M}}=0.3$, most of them at $z<1$. There are many fewer massive clusters at $z>0.5$ in the $\tau$ CDM model with $\Omega_{\mathrm{M}}=1$ because cluster evolution is so rapid in that case. The lower panel shows the numbers of clusters with $M_{200}>1 \times 10^{15} h_{70} M_{\odot}$. Differences between models with $\Omega_{\mathrm{M}} \approx 0.3$ but differing values of $\Omega_{\Lambda}$ and $w$ should be detectable in large cluster surveys containing $\sim 10^{4}$ clusters and extending to $z \sim 1$.

evolution that must be accounted for in precise cosmological measurements. Galaxy formation plays a more explicit role in the mass-richness and $M_{200}-L_{\mathrm{X}}$ relations, because the optical luminosities of galaxies evolve with time and because the physics of galaxy formation alters the $L_{\mathrm{X}}-T_{\mathrm{X}}$ relation (Sec. IV.C). Scatter in the massobservable relation might also be larger at higher redshifts, given that the proportion of relaxed clusters may well be smaller earlier in time.

As an example of how mass-observable evolution affects observations of mass-function evolution, consider its effects on X-ray surveys. The upper left of Figure 7 shows how the cluster mass function evolves for two dif- 
ferent cosmologies, a standard $\Lambda \mathrm{CDM}$ model $\left(\Omega_{\mathrm{M}}=0.3\right.$, $\left.\Omega_{\Lambda}=0.7, w=-1, \sigma_{8}=0.9\right)$ and a $\tau$ CDM model $\left(\Omega_{\mathrm{M}}=1.0, \Omega_{\Lambda}=0.0, \sigma_{8}=0.5, \Gamma=0.21\right)$ whose power spectrum has been adjusted by hand so that its shape is similar to that of the $\Lambda \mathrm{CDM}$ model, as required by observations of large-scale structure (Sec. III.C). Massfunction evolution is quite pronounced in both models but is far stronger in the $\tau$ CDM model.

Evolution in the mass-temperature relation weakens the observed amount of cluster evolution when cluster number density is plotted as a function of temperature. The upper right of Figure 7 shows the result of using a $M_{200}-T_{\text {lum }}$ relation with $T_{\text {lum }} / T_{200}=1$ and zero dispersion. Because clusters of a given mass are hotter at earlier times, the higher redshift curves have translated to higher temperatures, compared with their positions in the upper-left panel. Additional mass-temperature evolution exceeding that predicted by the virial theorem and corresponding to values of $T_{\mathrm{lum}} / T_{200}$ that increase with redshift, would further reduce the evolution, but there is currently no evidence for such evolution.

Redshift-dependent changes in the luminositytemperature relation can have additional evolutionsoftening effects. The $L_{\mathrm{X}} \propto T_{\text {lum }}^{3}$ power-law form of the relation appears to remain the same with redshift, but the amount of evolution in the normalization is uncertain. Early assessments suggested no evolution in the normalization (Borgani et al., 1999; Donahue et al., 1999; Mushotzkv and Scharf, 1997). The lower right of Figure 7 shows the evolution of cluster number density plotted against luminosity for a non-evolving normalization and $L_{\mathrm{X}}=6 \times 10^{44} h_{70}^{-2} \mathrm{erg} \mathrm{s}^{-1}\left(T_{\text {lum }} / 6 \mathrm{keV}\right)^{3}$, again with no dispersion. These curves differ from the temperature-function curves only in the labeling of the horizontal axis. More recent results indicate that higher-redshift clusters of a given temperature are more luminous, with an evolving relation $L_{\mathrm{X}} \propto T_{\mathrm{lum}}(1+z)^{b_{L T}}$ where $0.5 \lesssim b_{L T} \lesssim 1.5$ (Ettori et al., 2003; Lumb et al., 2003; Vikhlinin et al., 2002, see Sec. IV.C.4). Figure 7 shows the same distribution functions for $b_{L T}=1.5$, at the high end of the suggested range. The extra redshift dependence in this case slides the high-redshift curves even further to the right, roughly compensating for all of the evolution in the underlying mass function.

These examples underscore the importance of constraining evolution in the mass-observable relations, even if the observables could be perfectly measured. In addition, one must bear in mind that the observations themselves can introduce spurious redshift dependences in the mass-observable relations, largely because distant clusters are more difficult to observe than nearby ones. Optical projection effects become progressively harder to deal with at high redshift, complicating observations of richness and velocity dispersion, observations of weak lensing have fewer background galaxies to measure, and the decline in X-ray surface brightness makes cluster temperature measurements more difficult. In many ways, the Sunyaev-Zeldovich effect is the most promising observ- able for characterizing high-redshift clusters because its magnitude does not depend on redshift (Sec. II.C).

There are three basic ways to deal with evolution in the normalization and perhaps the scatter of a massobservable relation:

- Assume a model for the evolution of the relation. Numerical simulations can be very helpful in providing a model for evolution of the normalization and scatter of mass-observable relations but give accurate results only if they include all the relevant physics.

- Assume a parametric form for the mass-observable relation inspired by theoretical models and try to calibrate it directly with observations. The normalization of the relation is usually assumed to be proportional to either $(1+z)$ or $H(z)$ raised to a power determined by a fit to observations. In practice, however, the mass-observable relations for distant clusters are not directly calibrated. What we have instead are relations that link one easily observed quantity, such as X-ray luminosity, to another that is more closely related to mass, like X-ray temperature or the weak-lensing distortion.

- Assume a parametric form for the mass-observable relation and apply self-calibration techniques to a large cluster survey to find the most likely parameters describing mass-observable evolution $(\mathrm{Hu}$, 2003; Levine et al., 2002; Majumdar and Mohr, 2003, 2004). Parameters involving redshiftdependent scatter in the relation can also be included in such an analysis. This technique is very promising but requires large surveys of distant clusters which are not yet in hand. Its accuracy is limited by the number of free parameters needed to describe the mass-observable relations - the fewer, the better. Having a realistic physical model for mass-observable evolution helps boost the accuracy achievable with self-calibration by reducing the number of unknown parameters.

A decade from now, when much larger cluster samples will be available, self-calibration will probably be the best way to calibrate the mass-observable relations. In the meantime, it would be wise to spend some effort on direct observational calibrations through cross-comparisons of multiple mass-tracing observables.

\section{Constraints on Dark Matter}

Surveys of distant clusters find modest evolution in their comoving number density fully consistent with cosmological models in which $\Omega_{\mathrm{M}} \approx 0.3$. Because the rate of mass-function evolution at moderate redshifts $(z \lesssim 0.5)$ is governed primarily by the overall matter density, this conclusion does not depend strongly on the value of $\Omega_{\Lambda}$. 


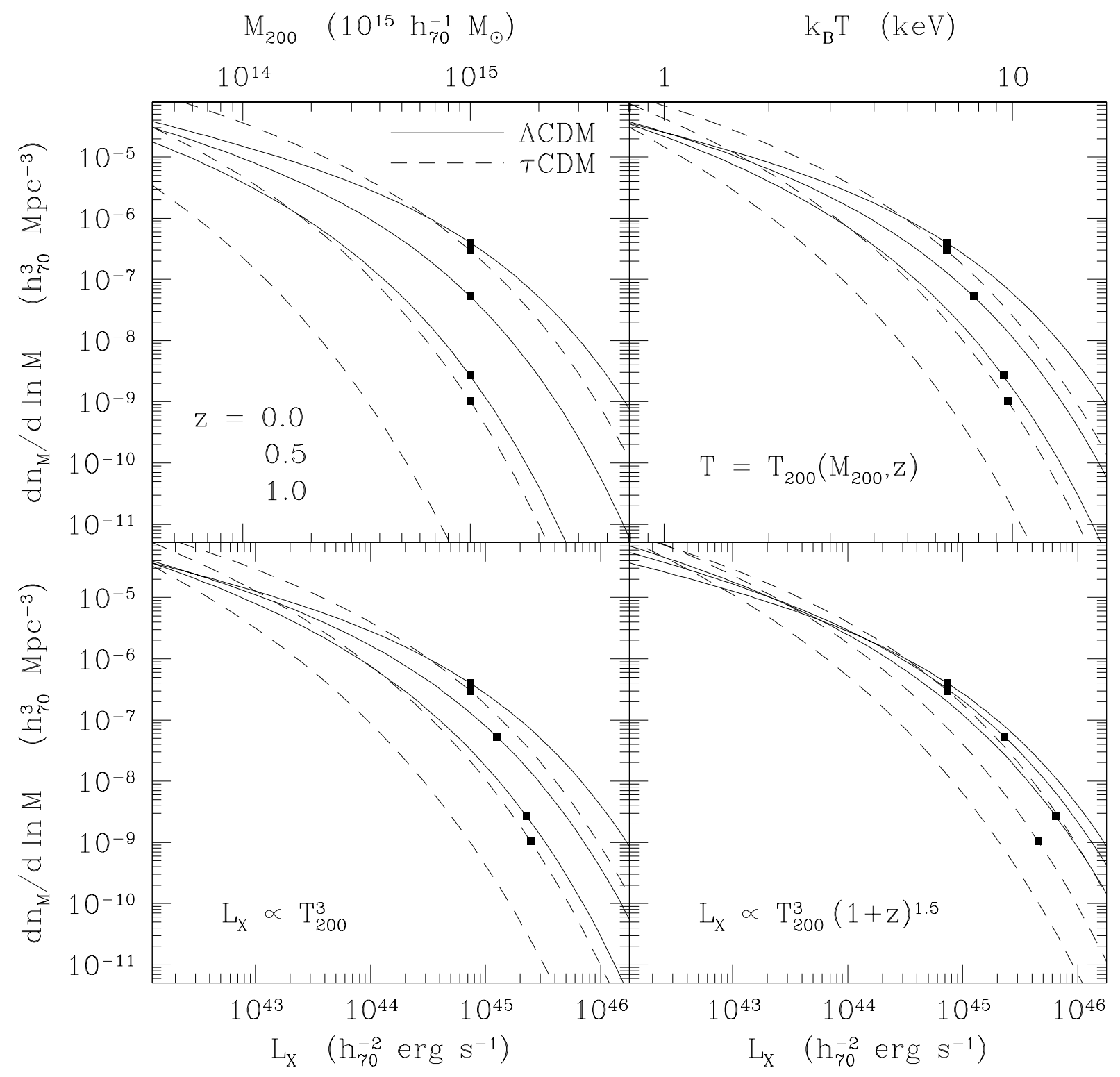

FIG. 7 Evolution of the cluster mass function and its manifestations in temperature and luminosity. The two models in this comparison are $\Lambda \mathrm{CDM}\left(\Omega_{\mathrm{M}}=0.3, \Omega_{\Lambda}=0.7, w=-1, \sigma_{8}=0.9\right)$ and $\tau \operatorname{CDM}\left(\Omega_{\mathrm{M}}=1.0, \Gamma=0.21, \sigma_{8}=0.5\right)$. Evolution of the mass function, shown at the upper left, is far more pronounced in $\tau$ CDM (dashed lines) than in $\Lambda$ CDM (solid lines) because it is so sensitive to the current matter density. Each set of three lines shows the differential mass function $d n_{M} / d \ln M$ at $z=0,0.5$, and 1.0, from top to bottom, and black squares show the value of the mass function at a fiducial mass of $10^{15} h_{70}^{-1} M_{\odot}$. The upper right panel shows the same mass functions plotted against temperature, assuming $T=T_{200}\left(M_{200}, z\right)$. Notice that the higher-redshift curves have shifted to the right, weakening the evolution in temperature space, because clusters of a given mass have higher temperatures at higher redshifts. In order to convert these curves to temperature functions, one would need to convolve them with the scatter in the mass-temperature relation and multiply by $d \ln M / d \ln T \approx 1.5$. The lower two panels show these same curves as a function of luminosity, assuming $L_{X}=\left(6 \times 10^{44} h_{70}^{-2} \mathrm{erg} \mathrm{s}^{-1}\right) T_{200}^{3}$ at $z=0$ and two different redshift dependences of the $L_{\mathrm{X}}-T$ relation. In the case without $L_{\mathrm{X}}-T$ evolution on the left-hand side, the curves are just relabeled versions of the ones in the upper-right panel. However, the strong $L_{\mathrm{X}}-T$ evolution in the right-hand panel $\left(L_{\mathrm{X}} \propto T^{3}(1+z)^{1.5}\right)$ shifts the three curves in the $\Lambda$ CDM case nearly on top of one another at $L_{\mathrm{X}} \approx 10^{44} h_{70}^{-1}$ erg s${ }^{-1}$. Convolving these curves with the dispersion in the mass-luminosity relation and multiplying by $d \ln M / d \ln L \approx 0.5$ converts them to luminosity functions. 


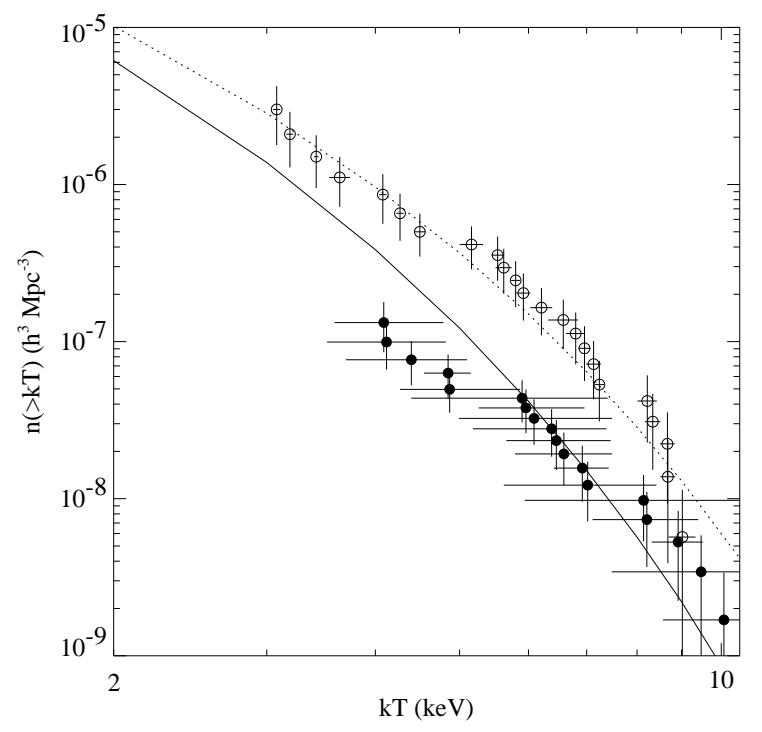

FIG. 8 Observed evolution in the integrated cluster temperature function $n(>k T)$ giving the comoving number density of clusters with temperatures exceeding $k T$. Open circles give the low-redshift temperature function from a sample of clusters with mean redshift $z=0.051$. Filled circles give the observed temperature function of clusters with a mean redshift of $z=0.429$. The data points in each case are correlated because $n(>k T)$ at a given temperature is a cumulative function depending on all data points at higher temperatures. Lines give the predicted temperature functions at $z=0.051$ (dotted line) and $z=0.429$ (solid line) for the best-fitting model: $\Omega_{\mathrm{M}}=0.28, \Omega_{\Lambda}=0.98, \sigma_{8}=0.68$. (Figure courtesy of Pat Henry.)

Here we focus on the constraints on $\Omega_{\mathrm{M}}$ derived from Xray surveys, whose observables $-L_{\mathrm{X}}, T_{\mathrm{X}}$, and baryonic mass - are related to to the spherical mass $M_{200}$ through simple parametric relations.

Evolution in the X-ray temperature function was first observed by Henrv (1997), who showed that the comoving number density of $\sim 5 \mathrm{keV}$ clusters at $z \sim 0.35$ was only slightly smaller than it is today. Assuming standard evolution of the mass-temperature relation, Eke et al. (1998) derived matter-density constraints $\Omega_{\mathrm{M}}=0.38 \pm 0.2$ for $\Omega_{\Lambda}=1-\Omega_{\mathrm{M}}$ and $\Omega_{\mathrm{M}}=0.44 \pm 0.2$ for $\Omega_{\Lambda}=0$ from these data using a maximum likelihood analysis to take full advantage of the sparse temperature data. More conservative analyses that simply counted clusters hotter than a given temperature found weaker constraints (Viana and Liddle, 1999). Henrv (2000) provides a complete discussion of the cluster temperature data and the maximum-likelihood analysis technique.

Temperature measurements of a handful of hot clusters at higher redshifts have shown that the rate of cluster temperature evolution remains modest at higher redshifts (Donahue, 1996; Donahue et al., 1998, 1999; Henrv, 2000). The comoving number density of $\gtrsim 8 \mathrm{keV}$ clusters at $z \sim 0.5-0.8$ is no less than about one tenth of its current value, in strong disagreement with the standard expectation in an $\Omega_{\mathrm{M}}=1$ universe (see Figure (7). Including these hot, distant clusters in the analysis further strengthens the constraints on the matter density, ruling out $\Omega_{\mathrm{M}}=1$ at the $3 \sigma$ level for standard mass-temperature evolution (Bahcall and Fan, 1998; Donahue and Voit, 1999; Donahue et al., 1998; Evrard et al., 2002). In order for such hot clusters to exist in a flat, matter-dominated universe, the masstemperature relation would have to evolve in a nonstandard way, with an increase in either the scatter or the normalization at $z \gtrsim 0.5$. Evrard et al. (2002) have shown that the $\tau \mathrm{CDM}$ mass function of Figure 7 is consistent with the temperature function observations only if the mass-temperature normalization factor $T_{1 \mathrm{um}} / T_{200}$ is $\sim 1.5$ times higher at $z \sim 0.5$ than at present. Such a big change seems unlikely in light of alternative observations of these hot, high-redshift clusters that agree with the large masses inferred from the standard normalization (Donahue et al., 1998; Luppino and Gioia, 1995; Tran et al., 1999).

Observations of evolution in the X-ray luminosity function have greater statistical power because many more clusters have known luminosities than have known temperatures, but uncertainties in luminosity-temperature evolution dilute the constraints they place on $\Omega_{\mathrm{M}}$. Many X-ray surveys have shown that the comoving number density of clusters at a given luminosity changes very little from redshift $z \sim 0.8$ to the present for $L_{\mathrm{X}} \lesssim$ $10^{44} \mathrm{erg} \mathrm{s}^{-1}$; significant evolution is seen only for clusters with $L_{\mathrm{X}} \gtrsim 10^{45} \mathrm{erg} \mathrm{s}^{-1}$ (Mullis et al., 2004; Rosati et al., 2002). Evolution this mild is generally expected in cosmological models with $\Omega_{\mathrm{M}} \approx 0.3$. Strong evolution in the luminosity-temperature relation must occur in models with $\Omega_{\mathrm{M}}=1$ for the observed evolution in the luminosity function to be so weak (see Figure 77). An extensive analysis by Borgani et al. (2001) of luminosity-function evolution in the ROSAT Deep Cluster Survey, which extends to $z \sim 1$, indicates that $\Omega_{\mathrm{M}}=0.35_{-0.10}^{+0.13}$, where the error bars signify the $1 \sigma$ confidence interval. Models with $\Omega_{\mathrm{M}}=1$ fall outside the $3 \sigma$ confidence interval, even when the normalization of the luminosity temperature relation is allowed to vary with redshift as $L_{\mathrm{X}} \propto T_{\text {lum }}(1+z)$.

The evolution of the baryon mass function observed with X-ray telescopes agrees with the conclusions drawn from the luminosity and temperature functions. Vikhlinin et al. (2003) have measured the baryon mass function in a sample of clusters at $z \sim 0.5$, finding that the comoving number density of massive clusters at that redshift is roughly one tenth of the current value. This result implies $\Omega_{\mathrm{M}}=0.25 \pm 0.1$ ( $1 \sigma$ confidence interval) for $\Omega_{\Lambda}=1-\Omega_{\mathrm{M}}$.

Optical studies concur that cluster evolution has been relatively modest since $z \sim 0.5$, buttressing the conclusion that $\Omega_{\mathrm{M}}<1$. In fact, the evolution of optically selected clusters appears even milder than the evolution in X-ray selected clusters (e.g., Postman et al., 2002), which would imply an even smaller value of $\Omega_{\mathrm{M}}$. However, it is not yet clear how much of this discrepancy arises from 


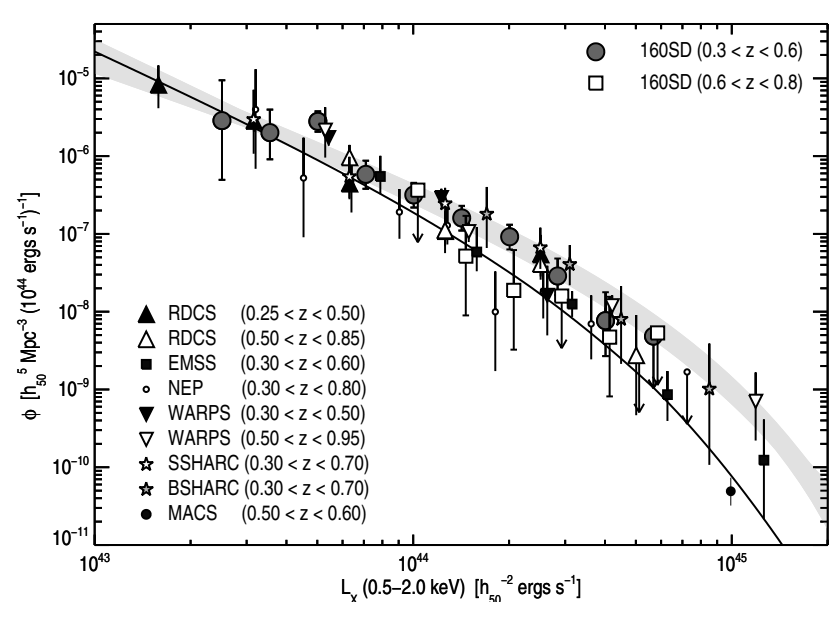

FIG. 9 Observed evolution in the cluster luminosity function. Many different cluster surveys spanning the range $0 \lesssim z \lesssim 1$ are shown on this figure. The vertical axis gives the luminosity functions derived from these surveys in terms of $\phi \equiv d n / d L_{\mathrm{X}}$, and the shaded region shows the luminosity function at $z \approx 0$. Significant evolution is seen only at $L_{\mathrm{X}} \gtrsim 10^{45} \mathrm{erg} \mathrm{s}^{-1}$, consistent with $\Lambda$ CDM models with a moderate amount of evolution in the $L_{\mathrm{X}}-T$ relation (see Figure 7 ) (Figure from Mullis et al. (2004).)

differences between the projected masses measured by optical surveys and the spherical masses measured by Xray surveys.

\section{Constraints on Dark Energy}

Existing cluster surveys, taken by themselves, do not yet place strong constraints on dark energy, but that situation is likely to change in the coming decade, with the advent of large, deep cluster surveys in the optical, Xray, and microwave bands. Currently, the most interesting information that clusters provide about dark energy comes from combining the results of cluster surveys with other information. If the overall geometry of the universe is indeed flat, as seems quite evident from the temperature patterns in the cosmic microwave background (e.g., Spergel et al., 2003), then the matter density inferred from clusters implies $\Omega_{\Lambda}=1-\Omega_{\mathrm{M}}=0.7 \pm 0.1$, in agreement with measurements of $\Omega_{\Lambda}$ from the supernova magnitude-redshift relation (Perlmutter et al., 1999; Riess et al., 1998). Geometrical arguments involving clusters provide weaker support for this conclusion. If the baryon fraction of clusters at a given temperature is assumed to remain constant with time, then the transverse sizes of clusters as a function of redshift can be used to constrain the geometry of the universe. Studies using such methods disfavor $\Omega_{\Lambda}=0$ (Arnaud et al., 2002; Mohr et al., 2000).

Large cluster surveys extending to $z \sim 1$ have the potential to place much stronger constraints on the darkenergy parameters $\Omega_{\Lambda}$ and $w$, independent of other infor- mation, as long as these surveys are large enough to permit self-calibration of the mass-observable relationships (Holder et al., 2001; Levine et al., 2002; Weller et al., 2002). The accuracy achievable with self-calibration depends critically on the nature of cluster evolution, because the self-calibration procedure requires evolution of the relevant mass-observable relation to be expressed in a parametric form. Constraints on the cosmological parameters are considerably weaker if the actual evolution does not follow the assumed parametric form. However, cross-calibration of mass-observable evolution through intensive supplementary observations of a small subset of the large survey restores much of the potential inherent in self-calibration (Majumdar and Mohr, 2003).

Including information on cluster bias inherent in a large cluster survey further tightens the constraints on dark energy. Because the tendency of clusters to cluster with one another depends in a simple way on the cosmological model (Sec. III.B.5), folding this information into the self-calibration procedure improves the accuracy with which cosmological parameters can be measured (Maiumdar and Mohr, 2004). Figure 10 shows how the estimated constraints on $\Omega_{\mathrm{M}}$ and $w$ tighten when information about cluster bias is added. It assumes that the universe is flat $\left(\Omega_{\Lambda}=1-\Omega_{\mathrm{M}}\right)$ and considers three different planned cluster surveys: two large SunyaevZeldovich surveys (SPT and Planck) and a large X-ray survey (DUET), each of which will find 20,000 to 30,000 cluster to $z \gtrsim 1$ (see Maiumdar and Mohr, 2004, for details). In the most optimistic cases, the parameters $\Omega_{\mathrm{M}}$, $\Omega_{\Lambda}$, and $w$ will be measured with $\sim 5 \%$ accuracy.

A large survey also minimizes the sample variance that arises from cluster bias (Evrard et al., 2002; $\mathrm{Hu}$ and Kravtsov, 2003). Because clusters tend to be clustered, the variance in the number of clusters within small sample volumes is larger than the gaussian expectation, adding systematic uncertainty to the measured mass function. This effect is generally not large for current cluster surveys but should be taken into account if one is designing a cluster survey for making highprecision cosmological measurements.

In summary, observations of cluster evolution already constrain the density of gravitating matter to be $\Omega_{\mathrm{M}} \approx$ $0.3 \pm 0.1$, meaning that $\Omega_{\Lambda} \approx 0.7 \pm 0.1$ if the universe is flat. Using this value of $\Omega_{\mathrm{M}}$ to break the $\Omega_{\mathrm{M}}-\sigma_{8}$ degeneracy leads to $\sigma_{8} \approx 0.7-1.0$, depending on the mass-temperature calibration. The major source of uncertainty in all these cosmological parameters comes not from the statistics of the survey but rather from uncertainties in the normalization and rate of evolution in the mass-observable relations. In order to better understand these relations and how they evolve, we need to know how galaxy formation affects the evolution of the stuff we can observe - the baryons in clusters. That is where we turn our attention next. 


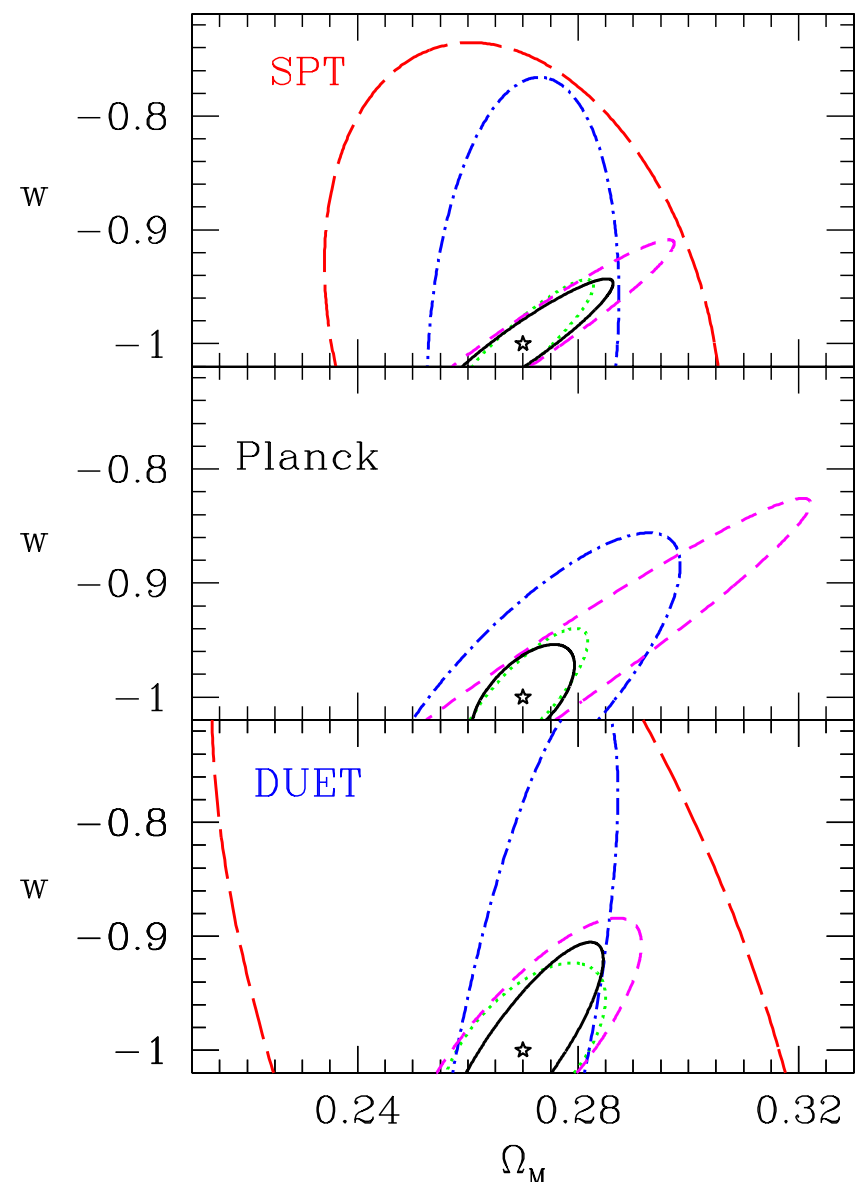

FIG. 10 Expected constraints on cosmological parameters from self-calibrated surveys. The SPT and Planck surveys will find 20,000 to 30,000 clusters through the S-Z effect. DUET is a proposed X-ray survey designed to find $\sim 20,000$ clusters. Dotted contours show the expected constraints on $w$ and $\Omega_{M}$ from self-calibration if redshift evolution of the cluster observables behaves exactly according to the standard scaling relations, allowing the redshift dependences of those scaling relations to be fixed. Long-dashed contours show how the constraints loosen when redshift evolution is determined as part of the self-calibration. Dot-dashed contours show how the contraints begin to tighten when information about cluster bias is included in the calibration. Solid contours show the best-case scenario in which the self-cailbration includes both information about cluster bias and supplementary follow-up calibrations of a small subset of the survey. (Figure from Maiumdar and Mohr (2004).)

\section{EVOLUTION OF THE BARYONIC COMPONENT}

Those who are cosmologists at heart are interested in how galaxy formation affects the intracluster medium primarily because they would like to know how to measure cluster masses more accurately. Those who are astronomers at heart are interested in the intracluster medium as well, but for them its main attraction is that the hot gas contains valuable information about the physical processes that govern galaxy formation. Clarifying the connections between galaxy formation and the massobservable relations is therefore important to both of these lines of research.

One of the nagging mysteries in our current picture of the universe is why so few of the universe's baryons have turned into stars (Cole, 1991; White and Frenk, 1991; White and Rees, 1978). Numerical simulations of cosmological structure formation that include baryonic hydrodynamics and the radiative cooling processes that lead to galaxy formation predict that $\gtrsim 20 \%$ of the baryons should have condensed into galaxies, but $\lesssim 10 \%$ have been found in the form of stars (e.g., Balogh et al., 2001b). Some form of feedback, involving supernovae and perhaps outflows from active galactic nuclei, seems to have stymied condensation, but we are still largely ignorant about how this feedback works.

Galactic winds like those observed from nearby starburst galaxies, in which multiple clustered supernovae are driving the powerful outflows, are likely to be important in regulating early star formation, but observational constraints on the mass and energy flux in such winds are sketchy at best (Heckman, 2002; Martin, 1999), particularly at high redshift (Adelberger et al., 2003; Pettini et al., 2001, 2000). These galactic winds presumably had a dramatic impact on the intergalactic medium and subsequent galaxy formation, with effects that may have persisted until the present day (e.g., Benson and Madau, 2003; Oh and Benson, 2003). Likewise, quasars and other forms of activity driven by black-hole growth in the nuclei of young galaxies may also have produced powerful outflows with lasting consequences for the intergalactic gas, but the energy input from these objects is still highly uncertain (Inoue and Sasaki, 2001; Nath and Rovchowdhurv, 2002; Scannapieco and Oh, 2004; Voit, 1994, 1996).

Unfortunately, the low-redshift intergalactic medium, where most of the universe's baryons are thought to reside, is notoriously hard to observe. Because the majority of this gaseous matter remains undetected, it is sometimes referred to as the "missing baryons" (e.g. Cen and Ostriker, 1999). A handful of quasars are bright enough beacons for probing the missing baryons via absorption-line studies with the ultraviolet spectrographs on the Hubble Space Telescope (e.g., Penton et al., 2002; Shull et al., 1996), and that number will increase if the Cosmic Origins Spectrograph is installed on Hubble. However, the inferences drawn from such studies depend critically on the uncertain heavy-element abundance and ionization state of these intergalactic clouds (e.g., Shull et al., 2003; Tripp et al., 2000).

Clusters of galaxies are still the only places in the universe where we have anything approaching a complete accounting of intergalactic baryons, their thermal state, and their heavy-element enrichment. Thus, observations of the intracluster medium can provide unique insights into the cooling and feedback processes that govern galaxy formation. In order to interpret the signatures of galaxy formation in the intracluster medium we need 
to understand how the thermodynamic properties of today's clusters are linked to the physics of the intergalactic baryons at $z \gtrsim 2$, the epoch of galaxy formation.

This section of the review discusses the current understanding of the interactions between galaxy formation and the intracluster medium, focusing in particular on how those interactions affect the mass-observable relations so crucial to cosmology. It begins by outlining the properties that clusters would have if radiative cooling of the universe's baryons and subsequent galaxy formation were suppressed. Because these properties do not agree with observations, radiative cooling and galaxy formation must somehow have altered the structure of the intracluster medium, with important consequences for the mass-observable relations. The middle of this section summarizes some of the recent progress that has been made in understanding the role of galaxy formation and its impact on the observable properties of clusters. It then concludes with a brief discussion of the existing constraints on baryon condensation in clusters.

\section{A. Structure Formation and Gravitational Heating}

People who study clusters of galaxies are sometimes asked how the X-ray emitting gas gets so hot. The answer to that question is simple. If radiative cooling is negligible, then gravitationally driven processes will heat diffuse gas to the virial temperature of the potential well that confines it. A tougher question would be to ask why the intracluster medium has the density that it does. In order to answer that question, one needs to know what produces the entropy of the X-ray emitting gas. Without galaxy formation in the picture, shocks driven by hierarchical structure formation are the only source of entropy for the intracluster medium, and this mode of entropy production leads to clusters whose density and temperature structures are nearly self-similar.

\section{Intracluster Entropy}

Entropy is of fundamental importance for two reasons: it determines the structure of the intracluster medium and it records the thermodynamic history of the cluster's gas. Entropy determines structure because highentropy gas floats and low-entropy gas sinks. A cluster's intergalactic gas therefore convects until its isentropic surfaces coincide with the equipotential surfaces of the dark-matter potential. Thus, the entropy distribution of a cluster's gas and the shape of the dark-matter potential well in which that gas sits completely determine the large-scale X-ray properties of a relaxed cluster of galaxies. The gas density profile $\rho_{g}(r)$ and temperature profile $T(r)$ of the intracluster medium in this state of convective and hydrostatic equilibrium are just manifestations of its entropy distribution.

This review adopts the approach of other work in this field and defines "entropy" to be

$$
K \equiv \frac{k_{\mathrm{B}} T}{\mu m_{p} \rho_{g}^{2 / 3}} .
$$

The quantity $K$ is the constant of proportionality in the equation of state $P=K \rho_{g}^{5 / 3}$ for an adiabatic monatomic gas, and is directly related to the standard thermodynamic entropy per particle, $s=k_{\mathrm{B}} \ln K^{3 / 2}+s_{0}$, where $s_{0}$ is a constant that depends only on fundamental constants and the mixture of particle masses. Another quantity frequently called "entropy" in the cluster literature is $S=k_{\mathrm{B}} T n_{e}^{-2 / 3}$. In order to avoid confusion with the classical definition of entropy, we will call this quantity $K_{e}$. For the typical elemental abundances in the intracluster medium, one can convert between these definitions using the relation

$$
\begin{aligned}
K_{e} & =k_{\mathrm{B}} T n_{e}^{-2 / 3} \\
& =960 \mathrm{keV} \mathrm{cm}^{2}\left(\frac{K}{10^{34} \mathrm{erg} \mathrm{cm}^{2} \mathrm{~g}^{-5 / 3}}\right) .
\end{aligned}
$$

A cluster achieves convective equilibrium when $d K / d r \geq$ 0 everywhere, and the entropy distribution that determines the gas configuration in this state can be expressed as $K\left(M_{g}\right)$, where the inverse relation $M_{g}(K)$ is the mass of gas with entropy $<K$.

Comparisons between the entropy distributions of clusters that differ in mass can be simplified by casting those distributions into dimensionless form (e.g., Voit et al., 2002). Combining the mean density of dark matter within the scale radius $r_{200}$, the global baryon fraction $f_{b}=\Omega_{\mathrm{b}} / \Omega_{\mathrm{M}}$, and the characteristic halo temperature $T_{200}$ gives the characteristic entropy scale

$$
\begin{aligned}
K_{200} & =\frac{k_{\mathrm{B}} T_{200}}{\mu m_{p}\left(200 f_{b} \rho_{\mathrm{cr}}\right)^{2 / 3}} \\
& =\frac{1}{2}\left[\frac{2 \pi}{15} \frac{G^{2} M_{200}}{f_{b} H(z)}\right]^{2 / 3} .
\end{aligned}
$$

For $f_{b}=0.022 h^{-2}$, this entropy scale reduces to

$$
\begin{aligned}
K_{e, 200}=362 & k_{\mathrm{B}} T_{\text {lum }} \mathrm{cm}^{2}\left(\frac{T_{200}}{T_{\text {lum }}}\right) \\
& \times\left[\frac{H(z)}{H_{0}}\right]^{-4 / 3}\left(\frac{\Omega_{\mathrm{M}}}{0.3}\right)^{-4 / 3} .
\end{aligned}
$$

Writing the entropy scale in this way makes explicit the fact that the observed temperature of a cluster is not necessarily a reliable guide to the characteristic entropy $K_{200}$ of its halo. If the intracluster medium of a real cluster is either hotter or cooler than $T_{200}$, then one must apply the correction factor $T_{200} / T_{\text {lum }}$ when computing the cluster's value of $K_{200}$.

\section{Entropy Generation by Smooth Accretion}

One way to approach the problem of gravitationally driven entropy generation is through spherically 
symmetric models of smooth accretion, in which gas passes through an accretion shock as it enters the cluster (e.g., Knight and Ponman, 1997; Tozzi and Norman, 2001; Voit et al., 2003). If the incoming gas is cold, then the accretion shock is the sole source of intracluster entropy. If instead the incoming gas has been heated before passing through the accretion shock, then the Mach number of the shock is smaller and the intracluster entropy level reflects both the amount of preheating and the production of entropy at the accretion shock.

Let us first consider the case of cold accreting gas, in which the pressure and entropy of the incoming gas are negligible. Suppose that mass accretes in a series of concentric shells, each with baryon fraction $f_{b}$, that initially comove with the Hubble flow as in the spherical collapse model of Sec. III.B.1 In this simple model, a shell that initially encloses total mass $M$ reaches zero velocity at the turnaround radius $r_{\mathrm{ta}}$ and falls back through an accretion shock at a radius $r_{\mathrm{ac}}$ in the neighborhood of the virial radius $r_{\mathrm{ta}} / 2$.

Because the cold accreting gas is effectively pressureless, the equations that determine the postshock entropy are

$$
\begin{aligned}
\dot{M}_{g} & =4 \pi r_{\mathrm{ac}}^{2} \rho_{1} v_{\mathrm{ac}} \\
v_{\mathrm{ac}}^{2} & =\frac{2 G M}{r_{\mathrm{ta}}} \\
k_{\mathrm{B}} T_{2} & =\frac{1}{3} \mu m_{p} v_{\mathrm{ac}}^{2} \\
\rho_{2} & =4 \rho_{1},
\end{aligned}
$$

where $\dot{M}_{g}=f_{b} \dot{M}$ is the gas accretion rate, $\rho_{1}$ is the preshock gas density, $T_{2}$ and $\rho_{2}$ are postshock quantities, and the accretion radius has been set to $r_{\mathrm{ac}}=r_{\mathrm{ta}} / 2$. Equations (69) and (70) are restatements of the jump conditions for strong shocks, assuming that the postshock velocity is negligible in the cluster rest frame (e.g., Cavaliere et al., 1997; Landau and Lifshitz, 1959), and equation (68) is exact only for cosmologies with $\Omega_{\Lambda}=0$.

The postshock entropy produced by smooth accretion of cold gas at time $t$ is therefore

$$
\begin{aligned}
K_{\mathrm{sm}} & =\frac{v_{\mathrm{ac}}^{2}}{3\left(4 \rho_{1}\right)^{2 / 3}} \\
& =\frac{1}{3}\left(\frac{\pi G^{2}}{f_{b}}\right)^{2 / 3}\left[\frac{d \ln M}{d \ln t}\right]^{-2 / 3}(M t)^{2 / 3} .
\end{aligned}
$$

Because the entropy generated at the shock front increases monotonically with time, such an idealized cluster never convects but rather accretes shells of baryons as if they were onion skins. The resulting entropy distribution in dimensionless form is

$$
\begin{aligned}
\frac{K_{\mathrm{sm}}\left(M_{g}\right)}{K_{200}}= & \frac{2}{3} \\
& \left(\frac{15}{2}\right)^{2 / 3}\left(H_{0} t_{0}\right)^{2 / 3} \\
& \times\left[\frac{d \ln \eta}{d \ln t}\right]^{-2 / 3}\left[\frac{\eta t(\eta)}{t_{0}}\right]^{2 / 3}
\end{aligned}
$$

where $\eta \equiv M_{g}(t) / f_{b} M_{200}\left(t_{0}\right)$ is effectively a radial coordinate corresponding to the amount of gas accreted by time $t$ divided by the amount accreted by the present time $t_{0}$. Given these assumptions, the entropy profile arising from smooth accretion of cold gas depends entirely on the mass accretion history $M(t)$, and the profiles of objects with similar accretion histories should be self-similar with respect to $K_{200}$.

This simple model yields entropy distributions whose overall shape agrees with cluster observations (Voit et al., 2003). The rate at which a cluster accretes matter through hierarchical structure formation depends on the growth function $D(t)$ and the powerlaw slope $\alpha_{M} \equiv d \ln \sigma^{-1} / d \ln M$ of the perturbation spectrum on the mass scale of the cluster: $M(t) \propto$ $[D(t)]^{1 / 2 \alpha_{M}}$ (Lacev and Cole, 1993; Voit and Donahue, 1998). Clusters ranging in mass from $10^{14} M_{\odot}$ to $10^{15} M_{\odot}$ grow roughly as $M(t) \propto t$ to $M(t) \propto t^{2}$ in the concordance model (Tozzi and Norman, 2001; Voit et al., 2003). Plugging these growth rates into equation (72) leads to entropy distributions between $K \propto M_{g}$ and $K \propto M_{g}^{4 / 3}$. Throughout much of a cluster, the gas mass encompassed within a given radius rises approximately linearly with radius (Sec. III.B.1), meaning that the $K(r)$ relation should be slightly steeper than linear. Numerical models of smooth accretion by Tozzi and Norman (2001) find $K(r) \propto r^{1.1}$. The entropy profile observed outside the core regions of clusters also obey $K(r) \propto r^{1.1}$ (Pratt and Arnaud, 2002, 2003), but the extent to which this agreement is coincidental is not clear.

If the accreting gas is not cold, then the intracluster entropy profile produced by smooth accretion has an isentropic core with an entropy level similar to the preshock entropy (Balogh et al., 1999; : Tozzi and Norman, 2001). A non-zero initial entropy level $K_{1}$ changes the coldaccretion model outlined above by altering the jump conditions represented by equations (69) and (70). When $K_{1}$ is no larger than the entropy generated at the accretion shock, then the entropy profile created by smooth accretion of warm gas can be closely approximated by adding $0.84 K_{1}$ to the entropy profile $K_{\mathrm{sm}}\left(M_{a}\right)$ from the coldaccretion case (Dos Santos and Doré, 2002; Voit et al., 2003). If $K_{1}$ is large compared with $K_{\mathrm{sm}}$, then the accretion shock is weak or non-existent, and accretion is nearly adiabatic, leading to an isentropic entropy profile with the constant value $K_{1}$.

\section{Entropy Generation by Hierarchical Merging}

In real clusters the accreting gas is lumpy, not smooth, which transforms the nature of entropy generation. Incoming gas associated with accreting sublumps of matter enters the cluster with a wide range of densities. There is no well-defined accretion shock but rather a complex network of shocks as different lumps of infalling gas mix with the intracluster medium of the main halo. Numerical simulations of this process beginning with cosmolog- 


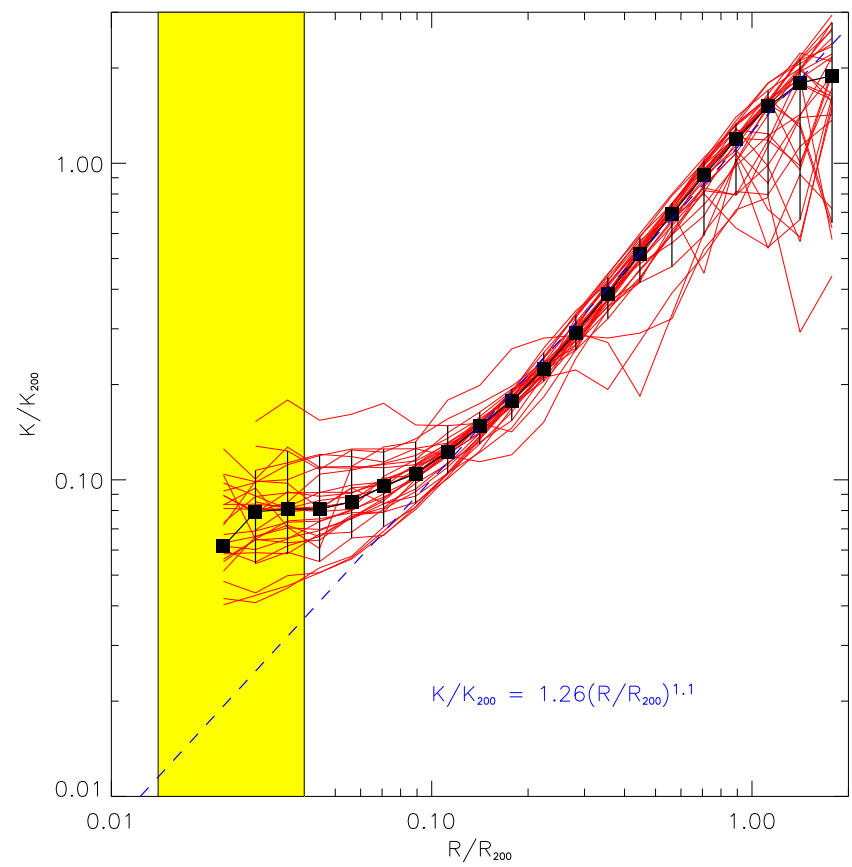

FIG. 11 Dimensionless entropy $K / K_{200}$ as a function of scale radius $r / r_{200}$ for 30 clusters simulated without radiative cooling or feedback. Black squares show the median profile, and the dashed line illustrates the power-law relation $K / K_{200}=1.26\left(r / r_{200}\right)^{1.1}$. Most of the entropy profiles shown lie close to this relation in the radial range $0.1 \lesssim r / r_{200} \lesssim 1.0$. At smaller radii, the entropy profiles generally flatten, and their dispersion increases. This flattening is likely to be a real effect, as it sets in well outside the shaded box showing the gravitational softening length of the simulation.

ical initial conditions produce clusters that have nearly self-similar entropy structure (e.g., Navarro et al., 1995), as expected from the scaling properties of hierarchical structure formation (Kaiser, 1986).

Figure 11] shows entropy profiles of 30 clusters generated with a numerical simulation of a $\Lambda \mathrm{CDM}$ cosmology including hydrodynamics but not radiative cooling (Kav, 2004; Voit et al., 2004). The masses of these clusters span more than a factor of 10 , but when their entropy profiles are divided by the appropriate value of $K_{200}$, they lie nearly on top of one another, at least outside the approximate core radius $0.1 r_{200}$. This result is not unique to the simulation method. All codes with sufficiently high resolution find that non-radiative clusters have approximately self-similar entropy structure, and consequently self-similar density and temperature structure (Frenk et al., 1999; Voit et al., 2004).

The self-similarity of the entropy profile in nonradiative clusters is a very useful point of comparison for sleuthing the effects of galaxy formation. Deviations from this baseline profile are likely to be due to a combination of radiative cooling and the feedback processes that ensue. Voit et al. (2004) find that a good representation of the baseline entropy profile produced outside the cores of clusters by hierarchical structure formation is given by the power law $K_{\mathrm{PL}}=(1.35 \pm$ $0.2) K_{200}\left(r / r_{200}\right)^{1.1}$. Specifying the baseline entropy profile within the cluster core $\left(<0.1 r_{200}\right)$ is more difficult both because there is more dispersion in that region among simulated clusters and because the results there depend somewhat on the hydrodynamical method used in the simulations.

Another notable aspect of self-similarity in nonradiative clusters is that the gas density profile and the dark-matter density profile outside $0.1 r_{200}$ have virtually identical shapes (Frenk et al., 1999; Navarro et al., 1995). This feature leads to another useful approximation to the entropy profiles of non-radiative clusters (Brvan, 2000). One can specify the gas density profile by assuming that it obeys an NFW density profile (Sec. III.B.2) with the same concentration as the dark matter and a total baryon mass $f_{b} M_{200}$ within $r_{200}$ and then compute the temperature profile that would keep the gas in hydrostatic equilibrium. The temperature and density profiles in this kind of model approximately obey the polytropic relation $T(r) \propto[\rho(r)]^{\gamma_{\text {eff }}-1}$, with $\gamma_{\text {eff }} \approx 1.1-1.2$ (Komatsu and Seliak, 2001; Voit et al., 2002). Combining them produces an alternative baseline entropy profile that depends on the concentration parameter $c_{200}$ of the dark-matter halo and that this review will denote as $K_{\mathrm{NFW}}(r)$.

Despite the complexity of the shock structure in hierarchical accretion, the numerically simulated entropy profiles are similar in shape to those created by smooth accretion models (Borgani et al., 2002a, 2001). However, these profiles have lower overall entropy levels than the smooth-accretion profiles (Voit et al., 2003). Figure 12 demonstrates this point by comparing the two approximations, $K_{\mathrm{PL}}$ and $K_{\mathrm{NFW}}$, of simulated non-radiative clusters with two entropy profiles drawn from smooth accretion models, one from the numerical computations of Tozzi and Norman (2001) and the other from equation (72) assuming $M \propto t^{3 / 2}$ and $H_{0} t_{0}=1$, which are reasonable assumptions for $\Lambda \mathrm{CDM}$ models.

The likely reason for this discrepancy is that smooth accretion maximizes entropy production because it minimizes the mean mass-weighted density of accreting gas (Ponman et al., 2003; Voit et al., 2003). Smoothing the accreting gas does not change the accretion velocity but does reduce the mean density of accreting gas lumps. Because postshock entropy scales as $v_{\mathrm{ac}}^{2} \rho_{1}^{-2 / 3}$, the mean entropy of lumpy accreted gas is therefore less than in the smooth-accretion case. This effect of smoothing might not be entirely academic, because the observed entropy profiles of low-temperature clusters show a similar entropy boost relative to the baseline profile (Voit and Ponman, 2003).

\section{Observed Entropy Profiles}

Astronomers have known for more than a decade that the structure of the intracluster medium in 


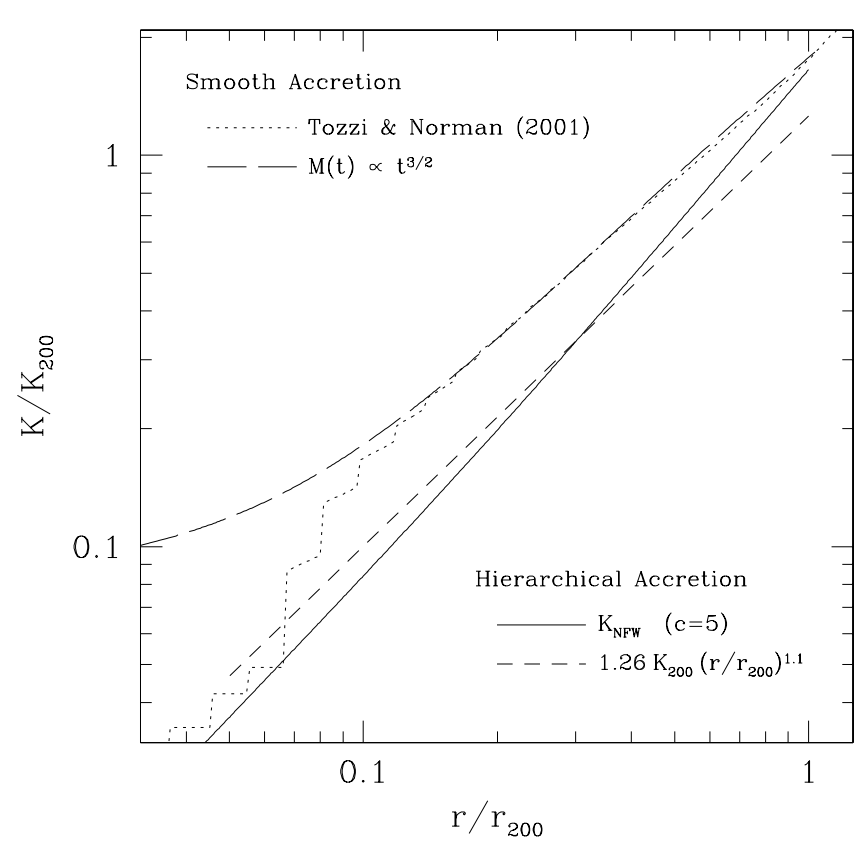

FIG. 12 Entropy profiles from smooth accretion and hierarchical accretion. Smoothing of the gas accreting onto a cluster boosts entropy production while maintaining the characteristic $K(r) \propto r^{1.1}$ entropy profile. The two lower lines illustrate approximate entropy profiles produced by hierarchical accretion, including the power-law expression from Figure 11 and the $K_{\mathrm{NFW}}$ model described in the text, with $c_{200}=5$. The two upper lines illustrate entropy profiles resulting from smooth accretion models, including profile computed by Tozzi and Norman (2001) for a $10^{15} h^{-1} M_{\odot}$ cluster with $300 \mathrm{keV} \mathrm{cm}^{2}$ of preheating and radiative cooling implemented (dotted line) and a profile computed from equation (172) and preheating amounting to $0.1 K_{200}$. The two smooth models run roughly parallel to the hierarchical accretion models but their normalizations are $\sim 1.5$ times higher.

real clusters cannot be self-similar because the luminosity-temperature relation of clusters does not agree with self-similar scaling (Edge and Stewart, 1991; Evrard and Henrv, 1991; Kaiser, 1991, Sec. III.C.3). Only within the last couple of years has the nature of that deviation from self-similarity become clear. Highquality cluster observations with the XMM-Newton satellite are showing that intracluster entropy profiles have the $K(r) \propto r^{1.1}$ shape characteristic of gravitational structure formation outside of the core, but the overall normalization of these profiles scales as $T_{\text {lum }}^{2 / 3}$ instead of $T_{\text {lum }}$, as in the baseline profiles (Pratt and Arnaud, 2003). Analyses of much larger cluster samples observed with earlier X-ray telescopes have arrived at the same conclusion. Instead of self-similarity with $K\left(r / r_{200}\right) \propto$ $T_{\text {lum }}$, Ponman et al. (2003) find altered similarity with $K\left(r / r_{200}\right) \propto T_{\text {lum }}^{2 / 3}$ at both the core radius $0.1 r_{200}$ and farther out in clusters, at the scale radius $r_{500} \approx 0.66 r_{200}$. The question to be answered is therefore how galaxy for- mation and feedback manage to produce such a shift in the overall normalization of cluster entropy profiles without substantially changing their shape.

\section{B. Galaxy Formation and Feedback}

In the decade since astronomers became aware of similarity breaking in clusters there have been many numerical simulations devoted to understanding it. Our understanding of this problem remains incomplete because including galaxy formation in cosmological models of cluster formation is a formidable computational challenge, requiring codes that simulate three-dimensional hydrodynamics spanning an enormous dynamical range in length scales and that track a large number of physical processes. The volume required to model a cosmologically significant sample of clusters is of order $10^{26} \mathrm{~cm}$ in linear scale, individual galaxies have sizes $\sim 10^{23} \mathrm{~cm}$, starforming regions within those galaxies can be as small as $10^{19} \mathrm{~cm}$, and the stars themselves are only $\sim 10^{11} \mathrm{~cm}$ in size. Sophisticated hydrodynamical techniques are now able to model the formation of the first stars from cosmological initial conditions (Abel, Bryan, \& Norman 2001), but are far from being able to track in detail the formation of an entire galaxy's worth of stars, let alone all the feedback processes that can occur.

For the time being, the difficulty of solving this problem from first principles means that modelers have to be selective about the physical processes and conditions that merit modeling. Important clues to what the essential processes are can be gleaned from the observed characteristics of clusters. This part of the review sifts through some of those clues, showing that radiative cooling is likely to be the process that sets the entropy scale of similarity breaking but that radiative cooling cannot act alone. Otherwise, too much baryonic matter would condense into stars and cold gas clouds.

\section{Preheating}

Early approaches to the problem of similarity breaking in clusters postulated that some sort of heating process imposed a universal minimum entropy — an "entropy floor" - on the intergalactic gas before it collected into clusters (Evrard and Henrv, 1991; Kaiser, 1991). Imposing a global entropy floor helps to bring the theoretical $L_{\mathrm{X}}-T_{\text {lum }}$ relation into better agreement with observations because this extra entropy makes the gas harder to compress in cluster cores, where entropy is smallest, particularly in the shallower potential wells of low-temperature clusters. This resistance to compression breaks cluster similarity by lowering the core density and therefore the $\mathrm{X}$-ray emissivity in low- $T$ clusters more than in high- $T$ clusters, thereby steepening the $L_{\mathrm{X}}-T_{\text {lum }}$ relation.

According to this preheating picture, the core entropy level and scaling relations of clusters should reflect the 
global entropy floor produced at early times. Initial measurements of entropy at the core radius $r_{0.1}$ demonstrated that low-temperature clusters had greater amounts of entropy than expected from self-similarity and suggested that the level of the entropy floor was $\sim 135 \mathrm{keV} \mathrm{cm}^{2}$ (Llovd-Davies et al., 2000; Ponman et al., 1999). This result matched well with numerical simulations of cluster formation with preheating levels of $50-100 \mathrm{keV} \mathrm{cm}^{2}$ that produced clusters with approximately the right $L_{\mathrm{X}^{-}}$ $T_{\text {lum }}$ relation (Bialek et al., 2001).

However, simple preheating now appears to be too crude an explanation for similarity breaking. In the preheating picture, low-temperature clusters should have large isentropic cores (Balogh et al., 1999; Tozzi and Norman, 2001), but this prediction disagrees with the observations showing that the shapes of cluster entropy profiles do not depend significantly on temperature (Sec. IV.A.4). In addition, the abundant evidence for intergalactic gas at $\lesssim 10^{5} \mathrm{~K}$ from quasar absorption line studies clearly shows that preheating cannot be global at $z \gtrsim 2$, and the preheating models themselves do not explain why the level of the entropy floor should be $\sim 135 \mathrm{keV} \mathrm{cm}^{2}$.

\section{Radiative Cooling}

In contrast, the observed entropy scale of similarity breaking emerges naturally from the process of radiative cooling. Intergalactic gas both inside and outside of clusters radiates thermal energy at a rate given by the cooling function $\Lambda_{c}(T)$, described in more detail in Sec. II.B.1 Cooling that radiates an energy $\Delta q$ per particle reduces the entropy by $\Delta \ln K^{3 / 2}=\Delta q / k_{\mathrm{B}} T$. Thus, the equation expressing these radiative losses can be written

$$
\frac{d K^{3 / 2}}{d t}=-\frac{3}{2} \frac{K_{c}^{3 / 2}(T)}{t_{0}}
$$

where

$$
K_{c}(T)=\left[\frac{2}{3}\left(\frac{n_{e} n_{p}}{\rho^{2}}\right) \frac{\left(k_{\mathrm{B}} T\right)^{1 / 2} \Lambda_{c}(T)}{\left(\mu m_{p}\right)^{1 / 2}}\right]^{2 / 3} t_{0}^{2 / 3}
$$

is the entropy level at which constant-density gas at temperature $T$ radiates an energy equivalent to its thermal energy in the time $t_{0}$. The latter formula reduces to

$$
K_{c}(T) \approx 81 \mathrm{keV} \mathrm{cm}^{2}\left(\frac{t_{0}}{14 \mathrm{Gyr}}\right)^{2 / 3}\left(\frac{T}{1 \mathrm{keV}}\right)^{2 / 3}
$$

when pure bremsstrahlung cooling is assumed.

The fact that the entropy threshold below which gas cools within the universe's lifetime is close to the entropy floor inferred from clusters with $\sim 2 \mathrm{keV}$ temperatures suggests that radiative cooling sets the entropy scale for similarity breaking (Voit and Brvan, 2001). Voit and Ponman (2003) further quantify this point. Figure 13 shows how entropy measurements at $0.1 r_{200}$ in a

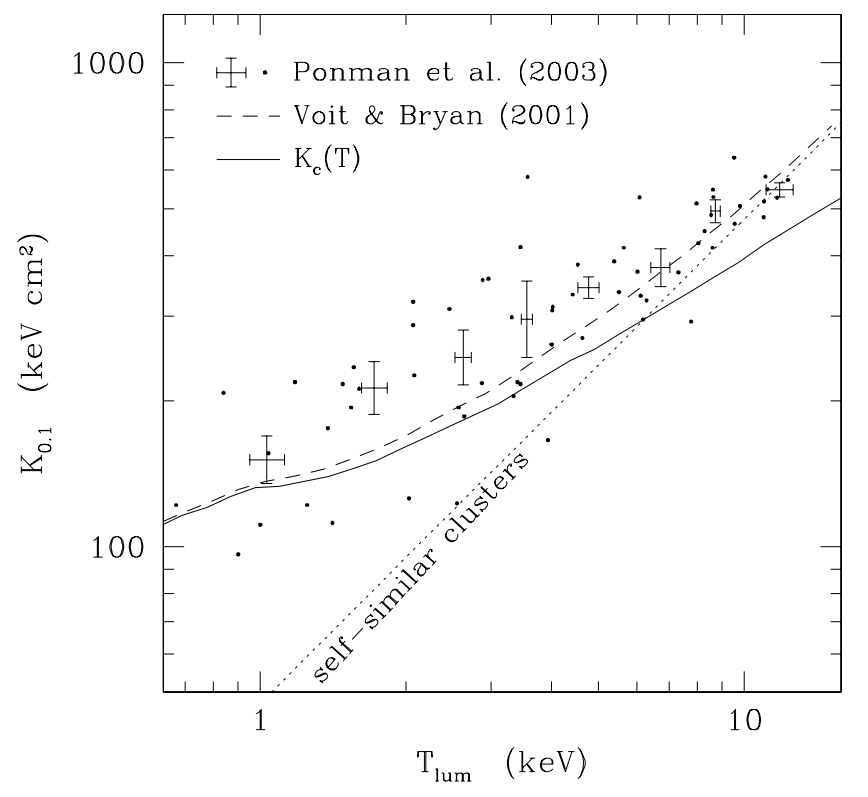

FIG. 13 Comparison between entropy measured at $0.1 r_{200}$ and the cooling threshold in a large sample of clusters. Small points show the entropy $K_{0.1}$ measured at $0.1 r_{200}$ in a sample of 64 clusters, and points with error bars show the mean entropy measurement in temperature bins of eight clusters each. The dotted line gives the mean entropy predicted by simulations of clusters without radiative cooling of feedback. The solid line shows the value of the cooling threshold $K_{c}(T)$ computed for heavy-element abundances 0.3 times their solar values and $t_{0}=14 \mathrm{Gyr}$. The dashed line shows the entropy predicted at $0.1 r_{200}$ by the simple analytical model of Voit and Brvan (2001).

large sample of clusters (Ponman et al., 2003) compare with the cooling threshold $K_{c}(T)$ for gas with heavyelement abundances equal to $30 \%$ of their solar values relative to hydrogen. Both the measured core entropies and the entropy threshold for cooling scale as $T^{2 / 3}$, and they are approximately equal, although the scatter in the data is quite significant.

Section IV.C.1 below shows that radiative cooling also accounts well for the scaling relations of global X-ray properties like $L_{\mathrm{X}}$ and $T_{\text {lum }}$ with mass. However, casting equation (75) in dimensionless form illustrates why at least some feedback must compensate for cooling:

$$
\frac{K_{c}(T)}{K_{200}} \approx 0.2(H t)^{2 / 3}\left[\frac{H(z)}{H_{0}}\right]^{2 / 3}\left(\frac{T}{1 \mathrm{keV}}\right)^{-1 / 3}
$$

The cooling threshold in low-temperature clusters at the present time is $\sim 20 \%$ of the characteristic entropy $K_{200}$ and greater than that if emission-line cooling from heavy elements is included. At earlier times, the dimensionless cooling threshold is even higher, meaning that a large proportion of the baryons belonging to the progenitor objects that ultimately assembled into presentday clusters should have condensed into stars or cold gas clouds. This is one of the manifestations of the 
classic overcooling problem of hierarchical galaxy formation (Blanchard et al., 1992; Cole, 1991; White and Rees, 1978). Because the observed mass ratio of stars to hot gas in clusters is only about $10 \%$ (Sec. IV.D), wholesale baryon condensation doesn't seem to have happened.

Recognition of this overcooling problem led Voit and Brvan (2001) to propose a way for radiative cooling to determine the entropy scale of similarity breaking without acting alone. The basic idea is that gas with entropy less than $K_{c}(T)$ cannot persist indefinitely. It must either cool and condense or be heated until its entropy exceeds $K_{c}(T)$. At any given time, feedback is triggered by condensing gas parcels with entropy less than the cooling threshold and acts until those parcels are eliminated by either cooling, heating, or some combination of the two. Thus, the joint action of cooling and feedback imprint an entropy scale corresponding to the cooling threshold, regardless of how strong the feedback is. This kind of effect has now been seen in a number of numerical simulations that include cooling and differing forms of feedback (Borgani et al., 2002a; Borgani et al., 2003; Davé et al., 2002; Kav et al., 2003; Valdarnini, 2003).

The fact that similarity breaking is not very sensitive to the efficiency of feedback is good news for cosmologists but bad news for astrophysicists. It offers hope that we can understand the mass-observable relations of clusters without solving all the messy astrophysical problems of feedback. Yet, it also implies that the mass-observable relations alone do not tell us much about the nature of that feedback. Instead, we must look to the spatially resolved entropy profiles of clusters (Kav, 2004; Voit and Ponman, 2003) and the ratio of condensed baryons to hot gas in clusters (Balogh et al., 2001b; Borgani et al., 2002a; Borgani et al., 2003; Kav et al., 2003).

\section{Feedback from Supernovae}

Supernovae are the most obvious candidate for supplying the feedback that suppresses condensation, but it is not clear that supernova heating and the galactic winds it drives can provide enough entropy to keep the fraction of condensed baryons below about 10\%. Heavy-element abundances in clusters imply that the total amount of supernova energy released during a cluster's history amounts to $\sim 0.3-1 \mathrm{keV}$ per gas particle in the intracluster medium (Finoguenov et al., 2001a; Pipino et al., 2002). The amount of energy input needed to explain the mass-observable relations while avoiding overcooling is $\sim 1 \mathrm{keV}$ (Tornatore et al., 2003; Voit et al., 2002; $\mathrm{Wu}$ et al., 2001), at the upper end of the range inferred from heavy elements, but the transfer of supernova energy to the intracluster medium must be highly efficient, which seems unlikely (Kravtsov and Yepes, 2000). Supernova energy would have to be converted to almost entirely to thermal energy with very little radiated away.

In order to avoid radiative losses, supernova heating must raise the entropy of the gas it heats to at least $100 \mathrm{keV} \mathrm{cm}^{2}$. An evenly distributed thermal energy input of order $1 \mathrm{keV}$ would therefore have to go into gas significantly less dense than $10^{-3} \mathrm{~cm}^{-3}$ to avoid such losses. Gas near the centers of present-day clusters, not to mention the galaxies where supernovae occur, is denser than that, particularly at earlier times when most of the star formation happened. Simulations that spread supernova feedback evenly therefore produce too many condensed baryons in clusters (Borgani et al., 2002a). Artificial algorithms that target supernova feedback at gas parcels that would otherwise cool are more successful at preventing overcooling (Kav et al., 2003). However, efforts to implement a more realistic version of targeted feedback in the form of galactic winds are still not entirely successful at preventing overcooling (Borgani et al., 2003).

It remains to be seen whether supernova feedback alone can account for the observed entropy profiles of clusters. Voit et al. (2003) and Ponman et al. (2003) have proposed that entropy input from galactic winds preceding the accretion of gas onto clusters could lead to a form of entropy amplification that would explain the observations. If galactic winds are strong enough to significantly smooth out the lumpiness of the local intergalactic gas, then the mode of accretion of this gas onto clusters will be closer to smooth accretion than to hierarchical accretion, thereby boosting the entropy generated through accretion shocks without changing the profile's characteristic shape. This effect is a plausible explanation for the altered similarity of the observed entropy profiles, but it has not yet been thoroughly tested in simulations. Intriguing results by Kav (2004) show that an extremely targeted feedback model, in which supernovae heat the local gas to $1000 \mathrm{keV} \mathrm{cm}^{2}$, successfully reproduces both the normalization and shape of the observed entropy profiles.

\section{Feedback from Active Galactic Nuclei}

If supernovae cannot prevent overcooling, then perhaps supermassive black holes in the nuclei of galaxies are what stop it Cavaliere et al., 2002; Valageas and Silk, 1999; Wu et al., 2001). The omnipresence of supermassive black holes at the centers of galaxies (Magorrian et al., 1998) and the excellent correlation of their masses with the bulge and halo properties of the host galaxy (Ferrarese and Merritt, 2000; Gebhardt et al., 2000) strongly suggest that the growth of black holes in the nuclei of galaxies goes hand-in-hand with galaxy formation. Furthermore, the centers of many clusters with low-entropy gas whose cooling time is less than the age of the universe also contain active galactic nuclei that are ejecting streams of relativistic plasma into the intracluster medium (Burns, 1990). It is therefore plausible that supermassive black holes at the centers of clusters provide feedback that suppresses further cooling whenever condensing intracluster gas accretes onto the 
central black hole.

Such a feedback loop is attractive and consistent with the circumstantial evidence, but the precise mechanism of heating remains unclear. The bubbles of relativistic plasma being inflated by the active galactic nuclei in clusters appear not to be expanding fast enough to shock heat the intracluster medium because the rims of the bubbles are no hotter than their surroundings (Fabian et al., 2000; McNamara et al., 2000). Also, if active galactic nuclei simply injected heat energy into the center of a cluster, then one would expect to see a flat or reversed entropy gradient in clusters with strong nuclear activity, indicating that convection is carrying heat outward. Instead, the entropy gradients in these cluster cores increase monotonically outward (David et al., 2001; Horner et al., 2004). One possibility is that heating is episodic (Kaiser and Binney, 2003) and that we have not yet found a cluster in the midst of an intense heating episode. Another is that heating is somehow spread evenly throughout the cluster core in a way that maintains the entropy gradient (Brüggen and Kaiser, 2002; Ruszkowski and Begelman, 2002). Yet another possibility is that bursts of relativistic plasma drive sound waves into the intracluster medium that eventually dissipate into heat (Fabian et al., 2003).

Unfortunately, the none of these heating mechanisms have yet been tested in the context of cosmological structure formation, so we do not know their overall impact on either baryon condensation or the global entropy profiles of clusters. Also, many aspects of the relationship between cosmology and nuclear activity in galaxies remain highly uncertain. A major role for quasar feedback is plausible. However, the connection between the growth of central black holes in galaxies and galaxy formation itself is not well understood, and the efficiency with which black holes convert accretion energy into outflows is unknown.

\section{Transport Processes}

Heat transport processes like thermal conduction and turbulent mixing may also mitigate radiative cooling because gas that condenses sets up a temperature gradient along which heat energy can flow. In gas without magnetic fields, electrons conduct heat along temperature gradients giving a heat flux $\kappa_{s} \nabla T$, with $\kappa_{s} \approx$ $6 \times 10^{-7} \mathrm{~T}^{5 / 2} \mathrm{erg} \mathrm{cm}^{-1} \mathrm{~s}^{-1} \mathrm{~K}^{-7 / 2}$ (Spitzer, 1962), the socalled Spitzer rate, valid when the scale length of the temperature gradient is longer than the electron mean free path. Clusters with central cooling times less than $H_{0}^{-1}$ indeed tend to have positive temperature gradients within the central $\sim 100 \mathrm{kpc}$, raising the possibility that heat conduction at least partially balances radiative losses. Many models for conduction in cluster cores have been developed (e.g., Bertschinger and Meiksin, 1986; Bregman and David, 1988; Rosner and Tucker, 1989; Sparks, 1992; Tucker and Rosner, 1983), but con- duction does not satisfactorily balance radiative cooling. Temperature-gradient observations are inconsistent with steady-state balance between cooling and conduction in a number of cluster cores (Horner et al., 2004; Voigt and Fabian, 2004). However, mixing of hot gas with cooler gas facilitated by intracluster turbulence (Kim and Naravan, 2003) or AGN activity (Brüggen and Kaiser, 2002) could enhance the effectiveness of heat conduction.

It is possible that cooling, conduction, feedback, and perhaps mixing as well are all needed for a complete solution that explains the observed core temperature gradients without overcooling. Conduction that balances cooling in a steady state has often been dismissed on the grounds that it is not stable enough to preserve the observed temperature and density gradients for periods of order $\gtrsim 1$ Gyr (Cowie and Binnev, 1977; Fabian, 1994). Because of conduction's extreme sensitivity to temperature, it is difficult for radiative cooling and conduction to achieve precise thermal balance with a globally stable temperature gradient (Bregman and David, 1988; Soker, 2003). On the other hand, conduction would have to be suppressed by at least two orders of magnitude for radiative cooling to produce the observed gradients (Binnev and Cowie, 1981; Fabian et al., 1981). Recent theoretical analyses of conduction have concluded that this level of suppression is unrealistically high (Malvshkin, 2001; Malyshkin and Kulsrud, 2001; Naravan and Medvedev, 2001). Combining cooling, conduction, and feedback offers a way out of this dilemma. Hybrid models in which conduction compensates for cooling in the outer parts of the core while feedback from an active galactic nucleus compensates for it in the inner parts have had some success in reproducing the observations (Brighenti and Mathews, 2003; Ruszkowski and Begelman, 2002).

\section{Galaxy Formation and Cluster Observables}

Earlier we saw that the X-ray properties of the self-similar clusters produced by purely gravitational structure formation do not agree with observations (Sec. IV.A ). Observed clusters of a given mass appear to be hotter than their theoretical counterparts and also less luminous, especially at the cool end of the cluster temperature range. Such disagreements have been worrisome to cosmologists who would like to understand what governs the cluster observables used to measure mass, but these problems are on their way to being solved. Both analytical work and hydrodynamical simulations performed during the last several years are showing that the observed $L_{\mathrm{X}}-T_{\text {lum }}, M_{200}-T_{\text {lum }}$, and $L_{\mathrm{X}}-M_{200}$ relations are natural outcomes of galaxy formation. Significant uncertainties remain, but the theoretical foundation for the mass-observable relations essential for probing cosmology with clusters is growing firmer. 


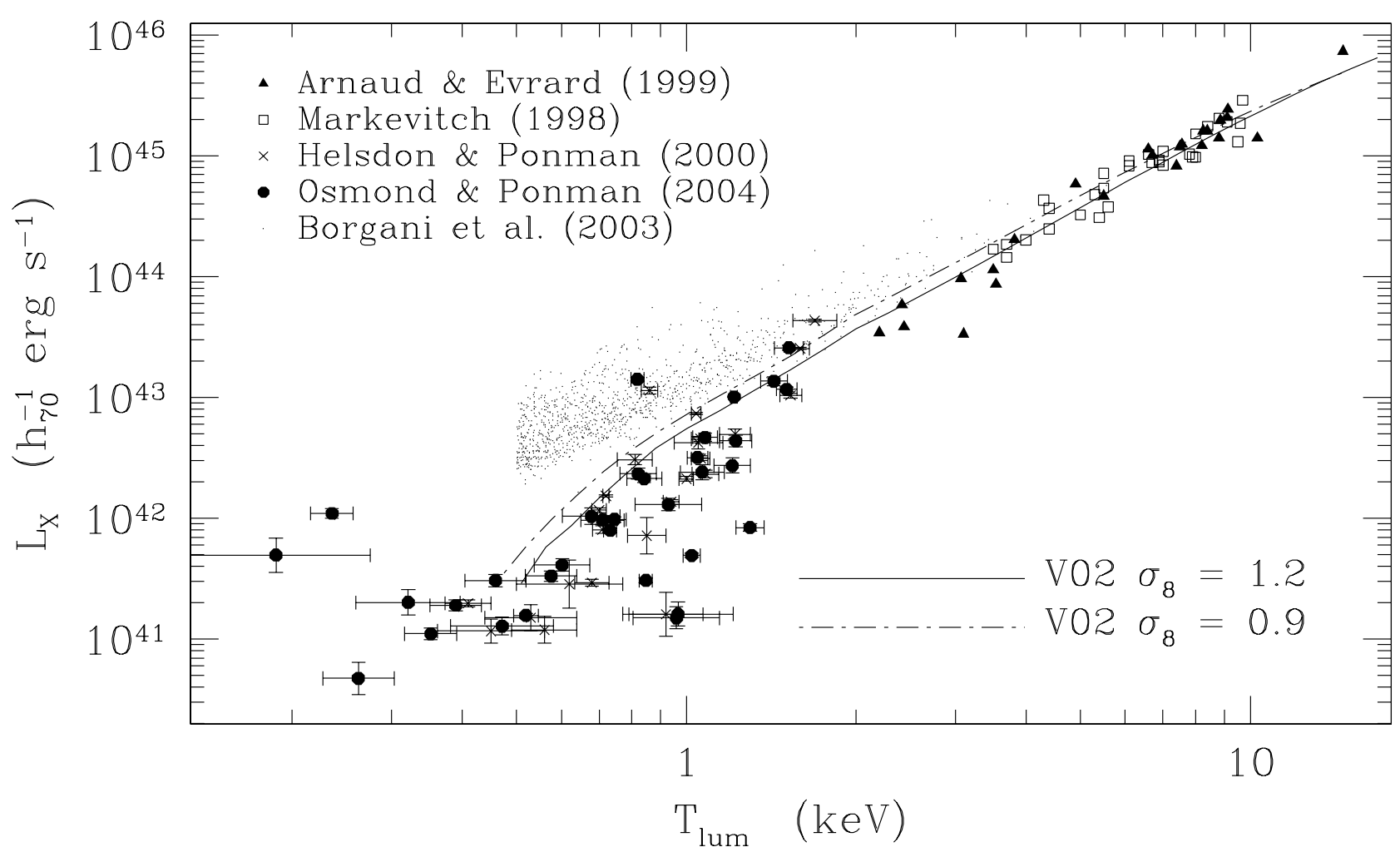

FIG. 14 Luminosity-temperature relation. Points show cluster data from Arnaud and Evrard (1999) (solid triangles) who avoided clusters with cool cores, cluster data from Markevitch (1998) (open squares) with cool cores excised, two sets of group data from Helsdon and Ponman (2000) (crosses) and Osmond and Ponman (2004) (solid octagons) that were not corrected for cool cores, and simulated clusters from Borgani et al. (2003) (small points). These simulations implement radiative cooling and supernova feedback in the form of galactic winds. Lines show modified-entropy models from Voit et al. (2002) with entropy truncated at the cooling threshold. There is a slight dependence on $\sigma_{8}$ in these models because higher values of $\sigma_{8}$ lead to dark-matter halos with more concentrated cores. Both the analytical and numerical models agree well with the data at $k_{B} T_{\text {lum }} \gtrsim 2 \mathrm{keV}$. Agreement is less good at lower temperatures, but the reasons for the disagreements are unclear. More feedback may be needed in the numerical models to suppress the luminosities, and the large scatter in the observations at $\lesssim 1 \mathrm{keV}$ may reflect a wide range in the effectiveness of feedback.

\section{Role of Cooling}

Radiative cooling turns out to the most important process to include. While it might seem paradoxical, allowing the intracluster medium to radiate thermal energy actually causes its luminosity-weighted temperature to rise. The reason for this behavior is that cooling selectively removes low-entropy gas from the intracluster medium, raising the mean entropy of what remains (Brvan, 2000; Knight and Ponman, 1997; Pearce et al., 2000). In nonradiative cluster simulations, the entropy of gas in the vicinity of the cluster core is below the cooling threshold $K_{c}$. This aspect of non-radiative models is unphysical, because gas with entropy less than $K_{c}$ would radiate an amount of energy greater than its total thermal energy content over the course of the simulations. When cooling is allowed to occur, this low-entropy core gas condenses out of the intracluster medium and is replaced by higher entropy core gas having a higher temperature, a lower density, and therefore a lower luminosity.
A simple analytical model illustrates the effect of the cooling threshold on the $L_{\mathrm{X}}-T_{\text {lum }}$ and $M_{200^{-}}$ $T_{\text {lum }}$ relations (Voit and Brvan, 2001; Voit et al., 2002; $\mathrm{Wu}$ and Xue, 2002). The model assumes that the intracluster entropy distribution in the absence of galaxy formation would be the $K_{\mathrm{NFW}}\left(M_{g}\right)$ distribution derived from the density profile of the dark matter. Because condensation and feedback both act to eliminate gas below the cooling threshold, the model simply truncates the entropy distribution at $K_{c}\left(T_{200}\right)$ and discards all the gas with lower entropy. One can interpret this gas removal either as condensation or as extreme feedback that heats the sub-threshold gas to a much higher entropy level. This cooling and feedback need not occur at the center of the cluster. In a hierarchical cosmology, much of the lowentropy gas cools, condenses into galaxies, and produces feedback long before the cluster is finally assembled.

Computing the hydrostatic configuration of the modified entropy distribution in the original dark-matter potential gives $L_{\mathrm{X}}$ and $T_{\mathrm{lum}}$ as a function of the mass $M_{200}$ 


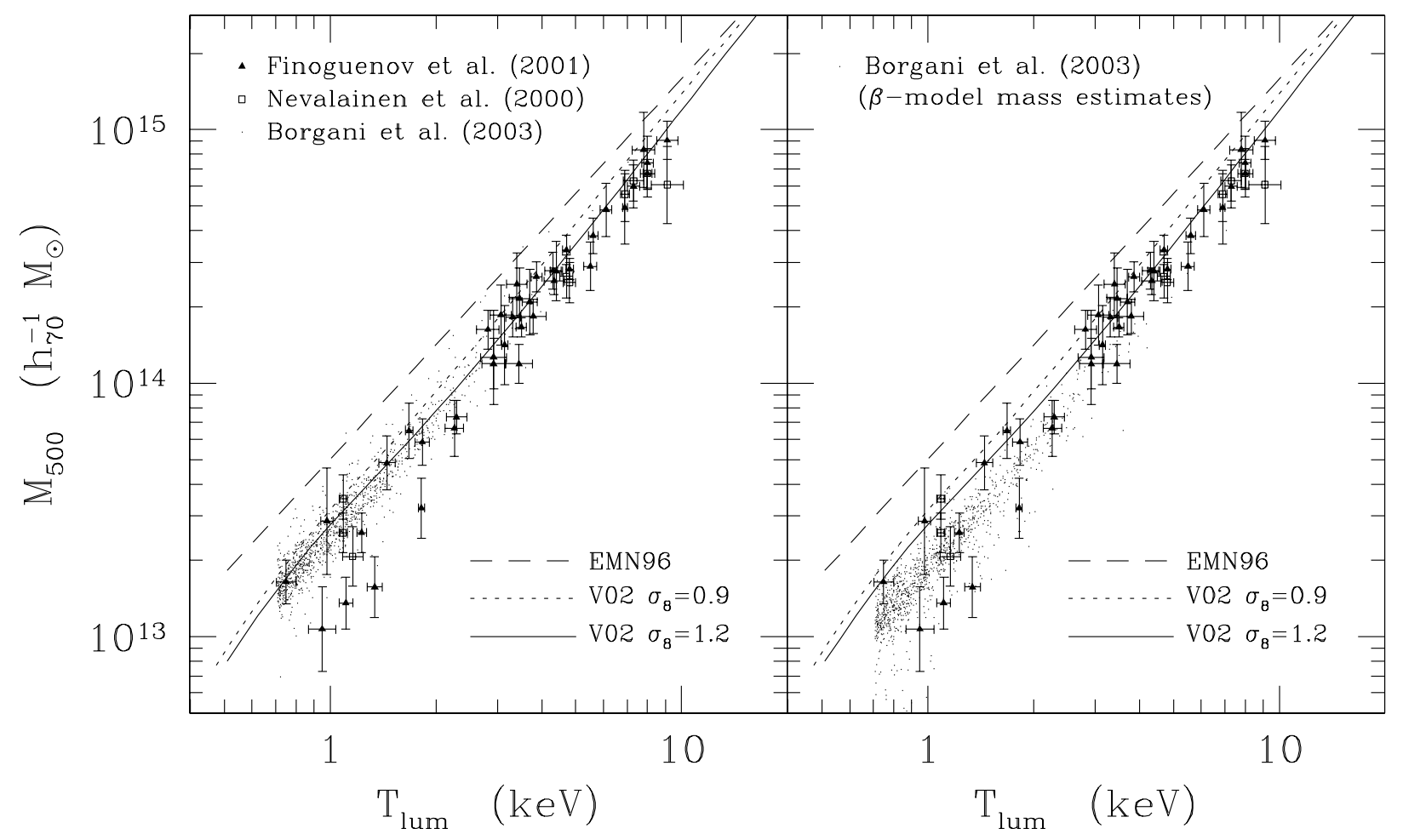

FIG. 15 Mass-temperature relation. Large points show cluster data from Finoguenov et al. (2001b) (solid triangles) and Nevalainen et al. (2000) (open squares), in which cluster masses were inferred from fitting polytropic beta models (see Sec. sec:tx). Dashed lines illustrate the $M_{500}-T_{\text {lum }}$ relation measured in clustered simulated without cooling and feedback by Evrard et al. (1996), which clearly disagree with the data points. The other lines show the $M_{500}-T_{\text {lum }}$ relations predicted by the analytical models of Voit et al. (2002), which agree much better with the data. There is a slight difference between models with $\sigma_{8}=0.9$ (dotted lines) and $\sigma_{8}=1.2$ because higher values of $\sigma_{8}$ lead to clusters with higher halo concentrations that produce slightly higher temperatures. Tiny points show data for clusters simulated by Borgani et al. (2003) with radiative cooling and feedback in the form of supernova-driven galactic winds. The left-hand panel uses the actual values of $M_{500}$, which agree with the analytical models. The right-hand panel uses values of $M_{500}$ inferred from fitting polytropic beta models to the observations, which underestimate true cluster masses, especially at low temperature, suggesting there may be a systematic observational bias in this method of mass measurement.

and concentration $c_{200}$ of the dark-matter halo. Figures [14 and [15] show that the resulting $L_{\mathrm{X}}-T_{\text {lum }}$ and $M_{200}-T_{\text {lum }}$ relations generally agree well with observations but may slightly overpredict $L_{\mathrm{X}}$ for objects cooler than $\sim 2 \mathrm{keV}$ and do not account for the large scatter at low temperatures. There are no free parameters in this model, other than the cosmological parameters, because the $M_{200}{ }^{-} c_{200}$ relation and the age of the universe used to compute $K_{c}$ depend only on cosmology, and the heavy-element abundance used to compute the cooling threshold is taken from observations.

Numerical simulations in which feedback is either weak or non-existent produce clusters whose properties are quite similar to the ones in this simple analytical model. Early numerical investigations of cooling in individual clusters gave inconclusive results (Lewis et al., 2000; Suginohara and Ostriker, 1998), but simulations by Muanwong et al. (2001) showed that adding cooling to a large-scale cluster simulation could give an $L_{\mathrm{X}}$ $T_{\text {lum }}$ like the observed one. Subsequent numerical work has confirmed that result (e.g., Borgani et al., 2002a; Davé et al., 2002; Kav et al., 2003; Valdarnini, 2003). Adding radiative cooling to the cosmological model produces good agreement with observations at all cluster temperatures $\gtrsim 2 \mathrm{keV}$.

Even when the simulations implement strong feedback, the X-ray scaling relations change remarkably little from the cooling-only case (Borgani et al., 2002a; Kav et al., 2003). The main effect on the $L_{\mathrm{X}}-T_{\text {lum }}$ relation of adding strong feedback to simulations that already include cooling is to slightly reduce the luminosity of cool $(\lesssim 2 \mathrm{keV})$ clusters, bringing them into better agreement with observations. This insensitivity to the efficiency of feedback is another strong indication that the cooling threshold governs the entropy scale for similarity breaking.

One point of disagreement between the analytical models, the simulations, and the observations concerns the central temperature gradient. Many observed clusters have a relatively small amount of gas in their cores whose cooling time is less than the age of the universe, and in 
those clusters the core temperature gradient is generally positive $(d T / d r>0)$. In the simple analytical models outlined above, no gas is allowed to be below the cooling threshold, resulting in a core that is nearly isentropic and thus has a negative temperature gradient $(d T / d r<0)$. Likewise, simulations with cooling and feedback also tend to have flat or negative temperature gradients in the neighborhood of the core radius ( $\sim 100 \mathrm{kpc})$.

This problem deserves attention because elevated core temperatures in models with cooling are what bring the theoretical $M_{200}-T_{\text {lum }}$ relation into agreement with observations. Making the analytical model slightly more realistic brings the predicted temperature gradient into better agreement with observations. The discontinuous cooling threshold applied by the simplest models is overly crude because it completely removes gas just below the threshold while gas just above the threshold does not cool at all. Instead, cooling acts upon the entropy distribution as described by equation (73). Voit et al. (2002) show that modifying the baseline profile $K_{\mathrm{NFW}}$ using this equation with $T=T_{200}$ for a time $t_{0}$ leads to an entropy distribution that reproduces the observed temperature gradients.

Simulations involving pure cooling do not agree with this result. The temperature-gradient discrepancy between analytical models and simulations in the purecooling case is still not understood, but may have something to do with the implicit stability of the cooling process in the analytical model. In that model the presentday intracluster medium is spherically symmetric with a positive entropy gradient, by definition, whereas thermal instabilities in the simulations that lead to a more heterogenous entropy pattern at each radius, may be at the root of the negative temperature gradient. Perhaps the observations are telling us that a stabilizing influence like conduction erases small-scale thermal instabilities without shutting off global cooling.

\section{Role of Feedback}

The primary role of feedback is to regulate how many baryons condense into stars and cold gas clouds. As mentioned in the discussion of cooling, strong feedback does not have a large effect on the $L_{\mathrm{X}}-T_{\text {lum }}$ relation, aside from a slight decrease in the luminosity of low-temperature clusters, as long as it is strong enough to shut off cooling in the gas parcels that it affects. However, moderate feedback that heats gas to $\lesssim 100 \mathrm{keV} \mathrm{cm}^{2}$ can boost $L_{\mathrm{X}}$ because it does not allow the core gas to cool but rather maintains it in an entropy state that allows it to radiate considerable thermal energy Kav et al. (2003).

Some preheating and feedback models adequately explain the scaling relations without explicitly including cooling (e.g., Babul et al., 2002; Balogh et al., 1999; Bialek et al., 2001; Tozzi and Norman, 2001). In these models, the minimum entropy level introduced by heating is typically a free parameter that is adjusted to give the best-fitting $L_{\mathrm{X}}-T_{\text {lum }}$ relation. The value of this bestfitting entropy level turns out to be $100-400 \mathrm{keV} \mathrm{cm}^{2}$, approximately corresponding to the level of the cooling threshold. This correspondence is consistent with the idea that the amount of heating needed to explain the mass-observable relation is determined by the need to shut cooling, in which case cooling still sets the entropy scale of similarity breaking, even when it is not explicitly included in the model (Voit et al., 2002).

From the standpoint of the mass-observable relations, the most important effect of feedback itself has to do with cluster richness. In both the simulations and the analytical models, pure cooling leads to a larger fraction of condensed baryons in cool clusters (Borgani et al., 2002a; Borgani et al., 2003; Davé et al., 2002; Muanwong et al., 2001; Voit et al., 2002), implying that these objects might have a higher star-to-baryon ratio and therefore a lower mass-to-light ratio. There are some observational indications that the ratio of stellar luminosity to mass in clusters is a function of mass (Lin et al., 2003), but not all such studies agree. This issue will need to be settled in order for optical richness measurements to deliver high-precision mass functions (Sec. IV.D).

\section{Role of Smoothing}

A full understanding of the $L_{\mathrm{X}}-T_{\text {lum }}$ relation may involve feedback indirectly, through its smoothing effects on the intergalactic medium (Sec. IV.A.3). If the observed preservation of $K(r) \propto r^{1.1}$ entropy profiles is indeed due to smoothing of the intergalactic medium followed by accretion onto clusters, then the presentday entropy profiles of clusters are evidence that galactic winds were widespread prior to the accretion of gas into today's clusters. Rather than just affecting the core entropy of clusters, a modest amount of entropy produced by early winds may have been amplified by smooth accretion, boosting the entire entropy profile by a common factor determined by the cooling threshold (Voit and Ponman, 2003). If that is indeed what happens, then it would explain the observed alteration of cluster similarity such that $K\left(r / r_{200}\right) \propto T_{\mathrm{lum}}^{2 / 3}$ (Ponman et al., 2003; Pratt and Arnaud, 2003), which leads directly to the relation $L_{\mathrm{X}} \propto T_{\text {lum }}^{3}\left(T_{200} / T_{\text {lum }}\right)^{1.5}$ for pure bremsstrahlung emission, in agreement with the observations.

\section{Predictions for Evolution}

Preheating, the cooling threshold, and the altered similarity indicative of smoothing affect the time-dependent behavior of the $L_{\mathrm{X}}-T_{\text {lum }}$ relation differently, offering a way to gather further information about their relative influence on cluster structure. Defining

$$
\hat{L}=\int_{0}^{r / r_{200}}\left(\frac{\rho_{g}}{200 f_{b} \rho_{\mathrm{cr}}}\right)^{2} \hat{r}^{2} d \hat{r}
$$


one can express the scaling of a cluster's integrated X-ray luminosity as

$$
\begin{aligned}
L_{\mathrm{X}} & \propto \Lambda_{c}\left(T_{\text {lum }}\right) M_{200} \rho_{\mathrm{cr}} \hat{L} \\
& \propto T_{\operatorname{lum}}^{2}\left(\frac{T_{200}}{T_{\text {lum }}}\right)^{3 / 2} H(z) \hat{L},
\end{aligned}
$$

where the first line assumes the cluster is approximately isothermal and the the second line is an approximation that assumes pure bremsstrahlung emission. The selfsimilar case,

$$
L_{\mathrm{X}} \propto T_{\operatorname{lum}}^{2} H(z)
$$

is well known to be a poor description of the data because its power-law slope at $z \approx 0$ is too shallow.

The modified-entropy models of Voit et al. (2002) show that enforcing a minimum core entropy level $K_{\min }$ breaks self-similarity in such a way that $\hat{L} \propto K_{\min }^{-3 / 2} T_{200}^{3 / 2} H^{-2}$, if $K_{\text {min }}$ is a significant fraction of the cluster's characteristic entropy $K_{200}$. In the pure preheating case, $K_{\min }$ is assumed to be independent of both cluster mass and of redshift, leading to

$$
L_{\mathrm{X}} \propto T_{\mathrm{lum}}^{3.5}\left(\frac{T_{200}}{T_{\text {lum }}}\right)^{3} \frac{1}{H(z)} .
$$

In other words, pure preheating steepens the $L_{\mathrm{X}}-T_{\text {lum }}$ a little more than necessary but causes high-redshift clusters to be less luminous than one would expect from their temperatures because the entropy floor $K_{\min }$ is a larger proportion of $K_{200}$ earlier in time. This prediction appears to conflict with recent observations indicating evolution in the opposite direction (e.g., Vikhlinin et al., 2002). Tying the minimum entropy scale to the cooling threshold $K_{c} \propto T_{\text {lum }}^{2 / 3} t^{2 / 3}$ helps to solve this problem because it leads to

$$
L_{\mathrm{X}} \propto T_{\operatorname{lum}}^{2.5}\left(\frac{T_{200}}{T_{\text {lum }}}\right)^{3} \frac{1}{H(z) t(z)} .
$$

In this case, a little bit of tilt in the $T_{\text {lum }} / T_{200}$ relation, consistent with observations (see Table I), is needed to sufficiently steepen the $L_{\mathrm{X}}-T_{\text {lum }}$ relation, and the sense of the evolution agrees with observations. In a $\Lambda \mathrm{CDM}$ universe, the redshift dependence of the luminosity normalization is $H^{-1} t^{-1} \sim(1+z)^{0.5} \sim H^{0.75}$ out to $z \sim 0.5$. Altered similarity linked to the cooling threshold is in better agreement with the slope but produces less evolution. Assuming density profiles that scale as $\rho_{g}\left(r / r_{200}\right) \propto\left(T_{\text {lum }} / K_{c}\right)^{3 / 2}$ yields

$$
L_{\mathrm{X}} \propto T_{\operatorname{lum}}^{3}\left(\frac{T_{200}}{T_{\text {lum }}}\right)^{3} \frac{1}{H^{3}(z) t^{2}(z)} .
$$

The normalization of luminosity in this relation varies as $H^{-3} t^{-2} \sim(1+z)^{0.3} \sim H^{0.5}$ to $z \sim 0.5$.

Observations of evolution in the luminositytemperature relation are not yet precise enough to distinguish between these latter two possibilities. The usual procedure is to compare the $L_{\mathrm{X}}-T_{\text {lum }}$ relation measured in a significantly redshifted cluster sample to the relation measured at $z \approx 0$. Vikhlinin et al. (2002) were the first to detect evolution, finding $L_{\mathrm{X}}\left(T_{\text {lum }}\right) \propto(1+z)^{b_{L T}}$ with $b_{L T}=1.5 \pm 0.3$, assuming a $\Lambda$ CDM cosmology. These authors compared the low-redshift sample of Markevitch (1998) to a collection of 22 clusters in the redshift range $0.4<z<0.8$. Lumb et al. (2003) found a similar amount of evolution, $b_{L T}=1.52_{-0.27}^{+0.24}$, using a smaller sample of eight clusters at $z \approx 0.4$, but not all studies find such strong evolution, which exceeds the predictions of the basic models outlined above. For example, Ettori et al. (2003) find $b_{L T}=0.62 \pm 0.28$ for a sample of 28 clusters at $z>0.4$ using the Markevitch (1998) sample as the low-redshift baseline and $b_{L T}=0.98 \pm 0.20$ relative to the Arnaud and Evrard (1999) low-redshift baseline. Furthermore, the strength of the evolution found by Ettori et al. (2003) becomes smaller for highredshift clusters, consistent with no evolution at all $\left(b_{L T}=0.04 \pm 0.33\right)$ when they include only their 16 clusters with $z>0.6$ in the comparison with the Markevitch sample. Apparently, there are some systematic uncertainties in these evolution measurements that need to be accounted for.

\section{Constraints on Baryon Condensation}

The ultimate test for feedback models is that they must account for both the proportion of condensed baryons to hot gas in clusters and any dependence of that proportion on cluster mass. In order to apply that test, we would like to have firm numbers for the amount of condensed baryons in clusters, but such measurements can be difficult. Even if the amount of starlight were perfectly measured, converting integrated starlight to stellar mass involves uncertain assumptions about both the starformation history of a cluster and the distribution function of stellar masses at birth, a quantity known as the initial mass function. Any variation in the star-formation history or initial mass function with cluster mass can lead to spurious systematic trends in the cluster mass function inferred from cluster richness.

Baryons contained in cold clouds are even harder to constrain because gaseous matter in this form can be nearly invisible, if it is sufficiently cold (Ferland et al., 1994, 2002). However, it seems unlikely that large amounts of baryons exist in such a form, at least in rich clusters. Adding the amount of baryons inferred from starlight to the amount of hot gas observed in rich clusters accounts for nearly all the baryons expected from the global ratio of baryons to dark matter, leaving little room left in the baryon budget for cold gas clouds.

The situation is less clear in lower-mass clusters and groups of galaxies, in which the proportion of hot gas to dark matter is significantly smaller. Summing the masses 
of stars and hot gas accounts for only about half the expected number of baryons in some cases, yet there is no observational evidence for large quantities of cold gas (e.g., Waugh et al., 2002). Circumstantial evidence argues against there being large reservoirs of cold baryons in groups. Presumably, the rich clusters in which we now see virtually all the baryons were hierarchically assembled from objects like the baryon-poor groups of galaxies we observe today. If large amounts of baryons in their higher-redshift counterparts were locked away in some cold, condensed form, then how were they released when these groups of galaxies merged to form large clusters?

A more complete accounting of intracluster baryons, especially in low-mass systems, is sorely needed in order test the various feedback models described in Sec. IV.B The rest of this section summarizes some of the recent work on constraining the amount of condensed intracluster baryons in the form of stars, the prospects for measuring baryon condensation through the S-Z effect, and X-ray observations of nearby clusters that may help solve the puzzles surrounding condensation and feedback.

\section{Mass and Light in Clusters}

Inferences of stellar mass from the observed starlight are generally based on a mass-to-light ratio expressed in solar units. That is, the mass-to-light ratio of the Sun in all wavebands equals unity. Because young stellar populations tend to emit large amounts of blue light that quickly dies out as the population ages, most recent assessments of the stellar mass in clusters have concentrated on measurements of infrared starlight in the $K$ band at roughly 2 microns. Observing starlight in this band minimizes the uncertainties owing to a cluster's star formation history. The old stellar populations characteristic of elliptical galaxies tend to have a $K$-band mass-to-light ratio $\Upsilon_{K} \approx 0.8 h_{70}^{-1}$, and mass-to-light ratios in spiral and irregular galaxies can be up to a factor of two smaller (Bell and de Jong, 2000). For the mix of galaxies seen in clusters, Lin et al. (2003) estimate that the mean mass-to-light ratio ranges from $\Upsilon_{K}=0.7 h_{70}^{-1}$ to $0.8 h_{70}^{-1}$ as cluster temperature climbs from $2 \mathrm{keV}$ to $10 \mathrm{keV}$. From this mass-to-light ratio, they infer that the fraction of intracluster baryons in stellar form is $f_{*} \approx 0.1$ for rich clusters (see also Balogh et al., 2001b). Notice that this value is about half that predicted by current simulations of cluster formation including strong feedback, a discrepancy that could become even larger with higher-resolution simulations (Borgani et al., 2003).

Many studies, but not all of them, suggest that the fraction of condensed baryons in stars may be a function of cluster mass. The ratio of $K$-band light to total cluster mass within $r_{500}$ found by Lin et al. (2003) is $\Upsilon_{K}=(47 \pm 3) h_{70}\left(M_{500} / 3 \times 10^{14} h_{70}^{-1} M_{\odot}\right)^{0.31}$, which translates to a temperature dependence $\Upsilon_{K} \propto T_{\text {lum }}^{0.5 \pm 0.1}$. The ratio of stellar mass to total mass in this study therefore ranges from $\sim 2.2 \%$ at $10^{14} h_{70}^{-1} M_{\odot}$ to $\sim 1.2 \%$ at $10^{15} h_{70}^{-1}, M_{\odot}$. Similar trends with shallower slopes are seen at other wavelengths. Bahcall and Comerford (2002) find the ratio of total mass to starlight in the heart of the visible spectrum ( $V$-band) is $\Upsilon_{V} \propto T_{\text {lum }}^{0.3 \pm 0.1}$. In blue light ( $B$-band), Girardi et al. (2002) find $\Upsilon_{B} \propto$ $M^{0.25}$. However, other studies have found no significant dependence on mass. According to Kochanek et al. $(2003)$ the $K$-band mass-to-light ratio inside $r_{200}$ scales as $\Upsilon_{K} \propto M_{200}^{-0.10 \pm 0.09}$.

\section{Intergalactic Stars}

Measurements of the total stellar luminosity in clusters generally focus on the light from galaxies, but what about stars that are not in galaxies? At least some of a cluster's stars float unmoored in the spaces between a cluster's galaxies (Ferguson et al., 1998). These stars are thought to have originated in galaxies but were later stripped from their homes by tidal forces during a close encounter with another galaxy. Current observational limits, however, indicate that no more than 10-20\% of a cluster's stars are outside of galaxies (Durrell et al., 2002), implying that failing to account for intergalactic stars does not lead to large errors in measured mass-tolight ratios.

\section{Global S-Z Effect}

If the baryons missing in low-mass clusters are not in condensed form, then they must be in the form of hot gas beyond the regions detectable with $\mathrm{X}$-ray telescopes. If that is indeed the case, then the best way of finding them may be through the Sunyaev-Zeldovich effect. Section II.C.1 showed that the integrated microwave distortion from a cluster scales with the electron temperature of the cluster and the overall mass in hot electrons. If a significant proportion of baryons have condensed, then the associated electrons are also locked away in cold clouds, where they don't contribute to the S-Z signal.

Simulations of cluster formation that include cooling indicate how the mean value of the $y$-distortion owing to clusters depends on cooling and feedback processes. Models by da Silva et al. (2001) produce $y=3.2 \times 10^{-6}$ in the non-radiative case, dropping to $y=2.3 \times 10^{-6}$ in the case of radiative cooling without feedback. The difference between the radiative case and non-radiative case is somewhat smaller when feedback is implemented. White et al. (2002) find $y=2.5 \times 10^{-6}$ in the nonradiative case and $y=2.1 \times 10^{-6}$ when both cooling and feedback are turned on. Testing for baryon condensation in this way may eventually be possible, but the mean value of the S-Z distortion is also very sensitive to other cosmological parameters, such as $\sigma_{8}$, which will have to be very well constrained before we can use the global $y$ parameter to test feedback models. 


\section{Cooling Flows in Clusters}

Cores of present-day clusters are among the best places in the universe to observe the interplay between condensation and feedback. Gas at the centers of many clusters can radiate an amount of energy equal to its thermal energy in less than a billion years, yet the majority of that gas is not condensing (see Donahue and Voit, 2004, for a recent review). Early interpretations of clusters with central cooling times less than the age of the universe suggested that the core gas should gradually condense and be replaced by the surrounding material in an orderly flow of cooling gas (Cowie and Binnev, 1977; Fabian and Nulsen, 1977; Mathews and Bregman, 1978). The mass condensation rates inferred from X-ray imaging ranged as high as $\sim 10^{2}$ to $10^{3} M_{\odot} \mathrm{yr}^{-1}$ implying that the cores of these "coolingflow" clusters should contain $\gtrsim 10^{12} M_{\odot}$ in the form of condensed baryons. However, exhaustive searches for this mass sink generally have not found stars forming at such a high rate (McNamara and O'Connell, 1989; O'Connell and McNamara, 1989), nor have they found sufficiently large collections of cold baryonic clouds to account for the deposited mass (Braine and Dupraz, 1994; McNamara and Jaffe, 1994; O'Dea et al., 1994, 1998; Voit and Donahue, 1995).

Now X-ray spectroscopy itself is showing that condensation proceeds at a considerably slower rate, if it happens at all. The central gas in clusters with short cooling times appears to reach temperatures $\sim T_{\text {lum }} / 2$, but very little X-ray line emission is seen from gas at $\lesssim T_{\text {lum }} / 3$ (Peterson et al., 2003, 2001). Some sort of heating mechanism seems to be inhibiting condensation below this temperature. There are plenty of candidates for resupplying the radiated heat energy - supernovae, outflows from active galactic nuclei, electron thermal conduction, and turbulent mixing have all been suggested (see Sec. IV.B - but there is still no consensus on the relative importance of these mechanisms.

A reduced amount of condensation still appears to be occurring. For example, plenty of circumstantial evidence links short central cooling times with star formation at the centers of clusters. Objects whose central cooling time is less than the age of the universe frequently contain emission-line nebulae whose properties suggest that they are energized primarily by hot, young stars (Johnstone et al., 1987; Voit and Donahue, 1997). Nebulae like these are never seen in clusters where the central cooling time is greater than the universe's age $\mathrm{Hu}$ et al., 1985). Also, objects with prominent nebulae tend to have abundant cool molecular hydrogen gas, the seed material for star formation (Donahue et al., 2000; Edge, 2001; Edge and Fraver, 2003). Efforts to estimate the star formation rate from the ultraviolet light emanating from the centers of clusters indicate that it may be consistent with the current upper limits on the condensation rate drawn from X-ray spectroscopy (McNamara et al., 2004).

An understanding of what regulates condensation and star formation at the centers of present-day clusters will help to solve more than just the overcooling problem of galaxy formation. It is also relevant to an aspect of bright galaxies that remains difficult to understand. The luminosity distribution function of galaxies cuts off very sharply at the high-luminosity end, far more sharply than called for in standard models of galaxy formation. Extremely powerful feedback can produce a sharp cutoff, but the amount of energy input required seems to implicate active galactic nuclei as the primary feedback source (e.g., Benson et al., 2003; Scannapieco and Oh, 2004). Alternatively, thermal conduction might produce a sharp cutoff because its efficiency rises so rapidly with temperature (Benson et al., 2003; Fabian et al., 2002). As the halo of a massive galaxy grows and its characteristic temperature rises through a critical threshold $\sim 10^{7} \mathrm{~K}$, conduction can strongly suppress further cooling and star formation, if it is not inhibited by magnetic fields. Detailed studies of cluster cores will be needed to test these possibilities. Early efforts are indicating that conduction might not be efficient enough to prevent overcooling (Dolag et al., 2004; Jubelgas et al., 2004).

\section{v. CONCLUDING REMARKS}

The next decade of research into cluster evolution promises to be very exciting. Large optical surveys like the Sloan Digital Sky Survey are greatly increasing the number of well-studied clusters of galaxies in the lowredshift universe. Deep surveys looking for the SunyaevZeldovich effect will be finding thousands of clusters to distances well beyond a redshift of $z=1$. The Chandra and XMM-Newton X-ray observatories are providing our most detailed look yet at the intracluster medium, its thermodynamical state, and some of the feedback processes that regulate condensation of intergalactic gas into galaxies and stars. Also, dedicated X-ray satellite missions to survey a large fraction of the sky for distant clusters are currently being planned.

Making the most of these opportunities will require cooperation between observers in those different wavebands, and theoretical modeling that closely links those cluster observables to cosmological parameters. Optical and infrared followup of S-Z surveys will be critical in order to determine the redshifts of the cluster candidates. X-ray followup of a subset of the S-Z clusters will also be necessary to establish how the thermodynamics of galaxy formation affects evolution of the mass-observable relations in the microwave band. Concentrated efforts to observe a calibration set of clusters in all of these wavebands will be very valuable in helping to establish how well the various observables trace mass and the scatter in each of these observables at a given mass.

If the $\Lambda \mathrm{CDM}$ concordance model is indeed a good description of the overall architecture of the universe and its initial perturbation spectrum, then the parameters describing the cosmological context in which galaxy for- 
mation happens ought to be quite precisely established within this decade. Studies of cluster evolution will be just one part of this overall effort, which also includes distance determinations to high-redshift supernovae, increasingly sensitive observations of the cosmic microwave background, and the mapping of large-scale structure. However, consistency between the dynamics of cluster evolution and the geometry of the universe, as measured with supernova and microwave observations, will stand as a particularly critical test of the overall model. With success, most of the remaining secrets about galaxy formation - other than what dark matter and dark energy actually are - will concern baryons and their complex cooling and feedback processes.

Our understanding of what baryons do is rapidly progressing, thanks in large part to large-scale cosmological simulations on massively parallel computers. Clusters and their evolution place unique constraints on those models because clusters are the only places in the universe where the majority of the baryons emit detectable radiation, revealing their thermodynamic state and elemental abundances. Galaxy formation has clearly left its mark in the intracluster medium, but we are just beginning to decipher what it has written there in the gases between the galaxies. Perhaps in ten more years there will be as much optimism about understanding the baryonic side of galaxy formation as there is now about the understanding the darker side.

\section{Acknowledgments}

Many colleagues have helped me to understand the issues covered in this review, particularly M. Donahue, G. Bryan, A. Evrard, J. Mohr, E. Ellingson, S. Borgani, T. Ponman, M. Balogh, R. Bower, C. Lacey, S. Kay, M. Postman, H. Ebeling, A. Fabian, C. Scharf, P. Tozzi, A. Finoguenov, S. Allen, B. McNamara, R. Mushotzky, C. Sarazin, R. Wechsler, H. Hoekstra, and M. White. Thanks also to A. Vikhlinin, P. Henry, and C. Mullis for providing some of the figures. Parts of this article were written at the very hospitable Aspen Center for Physics.

\section{References}

Abell, G. O., 1958, Astroph. J. Suppl. 3, 211.

Abell, G. O., H. G. Corwin, and R. P. Olowin, 1989, Astroph. J. Suppl. 70, 1.

Adelberger, K. L., C. C. Steidel, A. E. Shapley, and M. Pettini, 2003, Astroph. J. 584, 45.

Allen, S. W., and A. C. Fabian, 1998, Mot. Not. Roy. Ast. Soc. 297, L57.

Allen, S. W., R. W. Schmidt, H. Ebeling, A. C. Fabian, and L. van Speybroeck, 2004, eprint astro-ph/0405340.

Allen, S. W., R. W. Schmidt, and A. C. Fabian, 2001, Mot. Not. Roy. Ast. Soc. 328, L37.

Allen, S. W., R. W. Schmidt, and A. C. Fabian, 2002, Mot. Not. Roy. Ast. Soc. 334, L11.
Arnaud, M., N. Aghanim, and D. M. Neumann, 2002, Astron. Astroph. 389, 1.

Arnaud, M., and A. E. Evrard, 1999, Mot. Not. Roy. Ast. Soc. 305, 631.

Böhringer, H., C. A. Collins, L. Guzzo, P. Schuecker, W. Voges, D. M. Neumann, S. Schindler, G. Chincarini, S. De Grandi, R. G. Cruddace, A. C. Edge, T. H. Reiprich, et al., 2002, Astroph. J. 566, 93.

Babul, A., M. L. Balogh, G. F. Lewis, and G. B. Poole, 2002, Mot. Not. Roy. Ast. Soc. 330, 329.

Bacon, D. J., R. J. Massey, A. R. Refregier, and R. S. Ellis, 2003, Mot. Not. Roy. Ast. Soc. 344, 673.

Bahcall, J. N., and S. Tremaine, 1981, Astroph. J. 244, 805.

Bahcall, N. A., 1977, Ann. Rev. Astron. Astroph. 15, 505.

Bahcall, N. A., and J. M. Comerford, 2002, Astroph. J. Lett. 565, L5.

Bahcall, N. A., F. Dong, L. Hao, P. Bode, J. Annis, J. E. Gunn, and D. P. Schneider, 2003a, Astroph. J. 599, 814.

Bahcall, N. A., and X. Fan, 1998, Astroph. J. 504, 1.

Bahcall, N. A., and L. M. Lubin, 1994, Astroph. J. 426, 513.

Bahcall, N. A., T. A. McKay, J. Annis, R. S. J. Kim, F. Dong, S. Hansen, T. Goto, J. E. Gunn, C. Miller, R. C. Nichol, M. Postman, D. Schneider, et al., 2003b, Astroph. J. Suppl. 148, 243.

Bahcall, N. A., J. P. Ostriker, S. Perlmutter, and P. J. Steinhardt, 1999, Science 284, 1481.

Bahcall, N. A., and R. M. Soneira, 1983, Astroph. J. 270, 20.

Balogh, M. L., A. Babul, and D. R. Patton, 1999, Mot. Not. Roy. Ast. Soc. 307, 463.

Balogh, M. L., D. Christlein, A. I. Zabludoff, and D. Zaritsky, 2001a, Astroph. J. 557, 117.

Balogh, M. L., F. R. Pearce, R. G. Bower, and S. T. Kay, 2001b, Mot. Not. Roy. Ast. Soc. 326, 1228.

Bardeen, J. M., J. R. Bond, N. Kaiser, and A. S. Szalay, 1986, Astroph. J. 304, 15.

Bartelmann, M., and P. Schneider, 2001, Phys. Reports 340, 291.

Battye, R. A., and J. Weller, 2003, Phys. Rev. D 68, 083506.

Bell, E. F., and R. S. de Jong, 2000, Mot. Not. Roy. Ast. Soc. 312, 497.

Benson, A. J., R. G. Bower, C. S. Frenk, C. G. Lacey, C. M. Baugh, and S. Cole, 2003, Astroph. J. 599, 38.

Benson, A. J., and P. Madau, 2003, Mot. Not. Roy. Ast. Soc. 344, 835.

Bertschinger, E., 1985, Astroph. J. Suppl. 58, 39.

Bertschinger, E., and A. Meiksin, 1986, Astroph. J. Lett. 306, L1.

Bialek, J. J., A. E. Evrard, and J. J. Mohr, 2001, Astroph. J. 555, 597.

Binney, J., and L. L. Cowie, 1981, Astroph. J. 247, 464.

Binney, J., and S. Tremaine, 1987, Galactic dynamics (Princeton, NJ, Princeton University Press, 1987, 747 p.).

Birkinshaw, M., 1991, in Physical Cosmology, pp. 177-+.

Birkinshaw, M., 1999, Phys. Rept. 310, 97.

Birkinshaw, M., J. P. Hughes, and K. A. Arnaud, 1991, Astroph. J. 379, 466.

Biviano, A., 2000, in Constructing the Universe with Clusters of Galaxies.

Biviano, A., and M. Girardi, 2003, Astroph. J. 585, 205.

Blanchard, A., D. Valls-Gabaud, and G. A. Mamon, 1992, Astron. Astroph. 264, 365.

Bode, P., N. A. Bahcall, E. B. Ford, and J. P. Ostriker, 2001, Astroph. J. 551, 15.

Bond, J. R., S. Cole, G. Efstathiou, and N. Kaiser, 1991, 
Astroph. J. 379, 440.

Borgani, S., F. Governato, J. Wadsley, N. Menci, P. Tozzi, T. Quinn, J. Stadel, and G. Lake, 2002a, Mot. Not. Roy. Ast. Soc. 336, 409.

Borgani, S., M. Mezzetti, and R. Valdarnini (eds.), 2002b, Tracing Cosmic Evolution with Galaxy Clusters.

Borgani, S., P. Rosati, P. Tozzi, and C. Norman, 1999, Astroph. J. 517, 40.

Borgani, S., P. Rosati, P. Tozzi, S. A. Stanford, P. R. Eisenhardt, C. Lidman, B. Holden, R. Della Ceca, C. Norman, and G. Squires, 2001, Astroph. J. 561, 13.

Borgani, S., et al., 2003, astro-ph/0310794 .

Bower, R. G., 1991, Mot. Not. Roy. Ast. Soc. 248, 332.

Brüggen, M., and C. R. Kaiser, 2002, Nature (London) 418 , 301.

Braine, J., and C. Dupraz, 1994, Astron. Astroph. 283, 407.

Bregman, J. N., and L. P. David, 1988, Astroph. J. 326, 639.

Brighenti, F., and W. G. Mathews, 2003, Astroph. J. 587, 580.

Bryan, G. L., 2000, Astroph. J. Lett. 544, L1.

Bryan, G. L., and M. L. Norman, 1998, Astroph. J. 495, 80.

Bullock, J. S., T. S. Kolatt, Y. Sigad, R. S. Somerville, A. V. Kravtsov, A. A. Klypin, J. R. Primack, and A. Dekel, 2001, Mot. Not. Roy. Ast. Soc. 321, 559.

Buote, D. A., A. D. Lewis, F. Brighenti, and W. G. Mathews, 2003, Astroph. J. 595, 151.

Burles, S., K. M. Nollett, and M. S. Turner, 2001, Astroph. J. Lett. 552, L1.

Burns, J. O., 1990, Astron. J. 99, 14.

Carlberg, R. G., H. K. C. Yee, and E. Ellingson, 1997a, Astroph. J. 478, 462.

Carlberg, R. G., H. K. C. Yee, E. Ellingson, S. L. Morris, R. Abraham, P. Gravel, C. J. Pritchet, T. Smecker-Hane, F. D. A. Hartwick, J. E. Hesser, J. B. Hutchings, and J. B. Oke, 1997b, Astroph. J. Lett. 485, L13+.

Carlstrom, J. E., G. P. Holder, and E. D. Reese, 2002, Ann. Rev. Astron. Astroph. 40, 643.

Carlstrom, J. E., M. K. Joy, L. Grego, G. P. Holder, W. L. Holzapfel, J. J. Mohr, S. Patel, and E. D. Reese, 2000, Physica Scripta Volume T 85, 148.

Carroll, S. M., W. H. Press, and E. L. Turner, 1992, Ann. Rev. Astron. Astroph. 30, 499.

Cavaliere, A., and R. Fusco-Femiano, 1976, Astron. Astroph. 49, 137.

Cavaliere, A., A. Lapi, and N. Menci, 2002, Astroph. J. Lett. 581, L1.

Cavaliere, A., N. Menci, and P. Tozzi, 1997, Astroph. J. Lett. 484, L21+.

Cen, R., N. Y. Gnedin, L. A. Kofman, and J. P. Ostriker, 1992, Astroph. J. Lett. 399, L11.

Cen, R., and J. P. Ostriker, 1999, Astroph. J. 514, 1.

Cole, S., 1991, Astroph. J. 367, 45.

Cole, S., and N. Kaiser, 1989, Mot. Not. Roy. Ast. Soc. 237, 1127.

Collins, C. A., L. Guzzo, H. Böhringer, P. Schuecker, G. Chincarini, R. Cruddace, S. De Grandi, H. T. MacGillivray, D. M. Neumann, S. Schindler, P. Shaver, and W. Voges, 2000, Mot. Not. Roy. Ast. Soc. 319, 939.

Cowie, L. L., and J. Binney, 1977, Astroph. J. 215, 723.

da Silva, A. C., S. T. Kay, A. R. Liddle, P. A. Thomas, F. R. Pearce, and D. Barbosa, 2001, Astroph. J. Lett. 561, L15.

Dahle, H., K. Pedersen, P. B. Lilje, S. J. Maddox, and N. Kaiser, 2003, Astroph. J. 591, 662.

Dalton, G. B., S. J. Maddox, W. J. Sutherland, and G. Efs- tathiou, 1997, Mot. Not. Roy. Ast. Soc. 289, 263.

Davé, R., N. Katz, and D. H. Weinberg, 2002, Astroph. J. $\mathbf{5 7 9}, 23$.

David, L. P., W. Forman, and C. Jones, 1991, Astroph. J. 380, 39.

David, L. P., C. Jones, and W. Forman, 1995, Astroph. J. 445, 578 .

David, L. P., P. E. J. Nulsen, B. R. McNamara, W. Forman, C. Jones, T. Ponman, B. Robertson, and M. Wise, 2001, Astroph. J. 557, 546.

David, L. P., A. Slyz, C. Jones, W. Forman, S. D. Vrtilek, and K. A. Arnaud, 1993, Astroph. J. 412, 479.

Davis, M., G. Efstathiou, C. S. Frenk, and S. D. M. White, 1985, Astroph. J. 292, 371.

Davis, M., and P. J. E. Peebles, 1983, Astroph. J. 267, 465.

De Grandi, S., S. Ettori, M. Longhetti, and S. Molendi, 2003, eprint astro-ph/0310828.

De Grandi, S., and S. Molendi, 2002, Astroph. J. 567, 163.

Diaferio, A., and M. J. Geller, 1997, Astroph. J. 481, 633.

Dolag, K., M. Jubelgas, V. Springel, S. Borgani, and E. Rasia, 2004, Astroph. J. Lett. 606, L97.

Donahue, M., 1996, Astroph. J. 468, 79.

Donahue, M., J. Mack, C. Scharf, P. Lee, M. Postman, P. Rosati, M. Dickinson, G. M. Voit, and J. T. Stocke, 2001, Astroph. J. Lett. 552, L93.

Donahue, M., J. Mack, G. M. Voit, W. Sparks, R. Elston, and P. R. Maloney, 2000, Astroph. J. 545, 670.

Donahue, M., C. A. Scharf, J. Mack, Y. P. Lee, M. Postman, P. Rosati, M. Dickinson, G. M. Voit, and J. T. Stocke, 2002, Astroph. J. 569, 689.

Donahue, M., and G. M. Voit, 1999, Astroph. J. Lett. 523, L137.

Donahue, M., and G. M. Voit, 2004, in Carnegie Astrophysics Series, Vol. 3: Clusters of Galaxies: Probes of Cosmological Structure and Galaxy Evolution, edited by J. S. Mulchaey, A. Dressler, and A. Oemler, p. in press.

Donahue, M., G. M. Voit, I. Gioia, G. Lupino, J. P. Hughes, and J. T. Stocke, 1998, Astroph. J. 502, 550.

Donahue, M., G. M. Voit, C. A. Scharf, I. M. Gioia, C. R. Mullis, J. P. Hughes, and J. T. Stocke, 1999, Astroph. J. $\mathbf{5 2 7}, 525$.

Dos Santos, S., and O. Doré, 2002, Astron. Astroph. 383, 450.

Durrell, P. R., R. Ciardullo, J. J. Feldmeier, G. H. Jacoby, and S. Sigurdsson, 2002, Astroph. J. 570, 119.

Edge, A. C., 2001, Mot. Not. Roy. Ast. Soc. 328, 762.

Edge, A. C., and D. T. Frayer, 2003, Astroph. J. Lett. 594, L13.

Edge, A. C., and G. C. Stewart, 1991, Mot. Not. Roy. Ast. Soc. 252, 414.

Eisenstein, D. J., and W. Hu, 1998, Astroph. J. 496, 605.

Eisenstein, D. J., and W. Hu, 1999, Astroph. J. 511, 5.

Eke, V. R., S. Cole, and C. S. Frenk, 1996, Mot. Not. Roy. Ast. Soc. 282, 263.

Eke, V. R., S. Cole, C. S. Frenk, and J. P. Henry, 1998, Mot. Not. Roy. Ast. Soc. 298, 1145.

Eke, V. R., J. F. Navarro, and M. Steinmetz, 2001, Astroph. J. 554, 114.

Ettori, S., S. De Grandi, and S. Molendi, 2002, Astron. Astroph. 391, 841.

Ettori, S., P. Tozzi, S. Borgani, and P. Rosati, 2003, eprint astro-ph/0312239.

Ettori, S., P. Tozzi, and P. Rosati, 2003, Astron. Astroph. 398, 879 . 
Evrard, A. E., 1989, Astroph. J. Lett. 341, L71.

Evrard, A. E., 1990, Astroph. J. 363, 349.

Evrard, A. E., 1997, Mot. Not. Roy. Ast. Soc. 292, 289.

Evrard, A. E., 2004, in Carnegie Astrophysics Series, Vol. 3: Clusters of Galaxies: Probes of Cosmological Structure and Galaxy Evolution, edited by J. S. Mulchaey, A. Dressler, and A. Oemler, p. in press.

Evrard, A. E., and J. P. Henry, 1991, Astroph. J. 383, 95.

Evrard, A. E., T. J. MacFarland, H. M. P. Couchman, J. M. Colberg, N. Yoshida, S. D. M. White, A. Jenkins, C. S. Frenk, F. R. Pearce, J. A. Peacock, and P. A. Thomas, 2002, Astroph. J. 573, 7.

Evrard, A. E., C. A. Metzler, and J. F. Navarro, 1996, Astroph. J. 469, 494.

Fabian, A. C., 1994, Ann. Rev. Astron. Astroph. 32, 277.

Fabian, A. C., C. S. Crawford, A. C. Edge, and R. F. Mushotzky, 1994, Mot. Not. Roy. Ast. Soc. 267, 779.

Fabian, A. C., E. M. Hu, L. L. Cowie, and J. Grindlay, 1981, Astroph. J. 248, 47.

Fabian, A. C., and P. E. J. Nulsen, 1977, Mot. Not. Roy. Ast. Soc. 180, 479.

Fabian, A. C., J. S. Sanders, S. W. Allen, C. S. Crawford, K. Iwasawa, R. M. Johnstone, R. W. Schmidt, and G. B. Taylor, 2003, Mot. Not. Roy. Ast. Soc. 344, L43.

Fabian, A. C., J. S. Sanders, S. Ettori, G. B. Taylor, S. W. Allen, C. S. Crawford, K. Iwasawa, R. M. Johnstone, and P. M. Ogle, 2000, Mot. Not. Roy. Ast. Soc. 318, L65.

Fabian, A. C., L. M. Voigt, and R. G. Morris, 2002, Mot. Not. Roy. Ast. Soc. 335, L71.

Felten, J. E., R. J. Gould, W. A. Stein, and N. J. Woolf, 1966, Astroph. J. 146, 955.

Ferguson, H. C., N. R. Tanvir, and T. von Hippel, 1998, Nature (London) 391, 461.

Ferland, G. J., A. C. Fabian, and R. M. Johnstone, 1994, Mot. Not. Roy. Ast. Soc. 266, 399.

Ferland, G. J., A. C. Fabian, and R. M. Johnstone, 2002, Mot. Not. Roy. Ast. Soc. 333, 876.

Ferrarese, L., and D. Merritt, 2000, Astroph. J. Lett. 539, L9.

Fillmore, J. A., and P. Goldreich, 1984, Astroph. J. 281, 1.

Finoguenov, A., M. Arnaud, and L. P. David, 2001a, Astroph. J. 555, 191.

Finoguenov, A., A. Burkert, and H. Böhringer, 2003, Astroph. J. 594, 136.

Finoguenov, A., T. H. Reiprich, and H. Böhringer, 2001b, Astron. Astroph. 368, 749.

Fixsen, D. J., E. S. Cheng, J. M. Gales, J. C. Mather, R. A. Shafer, and E. L. Wright, 1996, Astroph. J. 473, 576.

Forman, W., E. Kellogg, H. Gursky, H. Tananbaum, and R. Giacconi, 1972, Astroph. J. 178, 309.

Freedman, W. L., B. F. Madore, B. K. Gibson, L. Ferrarese, D. D. Kelson, S. Sakai, J. R. Mould, R. C. Kennicutt, H. C. Ford, J. A. Graham, J. P. Huchra, S. M. G. Hughes, et al., 2001, Astroph. J. 553, 47.

Frenk, C. S., S. D. M. White, P. Bode, J. R. Bond, G. L. Bryan, R. Cen, H. M. P. Couchman, A. E. Evrard, N. Gnedin, A. Jenkins, A. M. Khokhlov, A. Klypin, et al., 1999, Astroph. J. 525, 554.

Gal, R. R., and L. M. Lubin, 2004, Astrophys. J. 607, L1.

Gebhardt, K., R. Bender, G. Bower, A. Dressler, S. M. Faber, A. V. Filippenko, R. Green, C. Grillmair, L. C. Ho, J. Kormendy, T. R. Lauer, J. Magorrian, et al., 2000, Astroph. J. Lett. 539, L13.

Gibson, B. K., and F. Matteucci, 1997, Mot. Not. Roy. Ast.
Soc. 291, L8.

Gilbank, D. G., R. G. Bower, F. J. Castander, and B. L. Ziegler, 2003, eprint astro-ph/0310809.

Girardi, M., S. Borgani, G. Giuricin, F. Mardirossian, and M. Mezzetti, 1998, Astroph. J. 506, 45.

Girardi, M., P. Manzato, M. Mezzetti, G. Giuricin, and F. Limboz, 2002, Astroph. J. 569, 720.

Gladders, M. D., and H. K. C. Yee, 2000, Astron. J. 120, 2148.

Gunn, J. E., 1977, Astroph. J. 218, 592.

Gunn, J. E., and J. R. I. Gott, 1972, Astroph. J. 176, 1.

Gursky, H., E. Kellogg, S. Murray, C. Leong, H. Tananbaum, and R. Giacconi, 1971, Astroph. J. Lett. 167, L81+.

Guth, A. H., and S.-Y. Pi, 1982, Physical Review Letters 49, 1110.

Haiman, Z., J. J. Mohr, and G. P. Holder, 2001, Astroph. J. 553, 545.

Hamilton, A. J. S., 2001, Mot. Not. Roy. Ast. Soc. 322, 419.

Harrison, E. R., 1970, Phys. Rev. D 1, 2726.

Heath, D. J., 1977, Mot. Not. Roy. Ast. Soc. 179, 351.

Heckman, T. M., 2002, in ASP Conf. Ser. 254: Extragalactic Gas at Low Redshift, pp. 292-+.

Heckman, T. M., L. Armus, and G. K. Miley, 1990, Astroph. J. Suppl. 74, 833.

Heisler, J., S. Tremaine, and J. N. Bahcall, 1985, Astroph. J. 298, 8 .

Helsdon, S. F., and T. J. Ponman, 2000, Mot. Not. Roy. Ast. Soc. 315, 356.

Henry, J. P., 1997, Astroph. J. Lett. 489, L1+.

Henry, J. P., 2000, Astroph. J. 534, 565.

Henry, J. P., and K. A. Arnaud, 1991, Astroph. J. 372, 410.

Herschel, F. W., 1785, Phil. Trans. 75, 213.

Hoekstra, H., 2001, Astron. Astroph. 370, 743.

Hoekstra, H., M. Franx, K. Kuijken, and G. Squires, 1998, Astroph. J. 504, 636.

Holder, G., Z. Haiman, and J. J. Mohr, 2001, Astroph. J. Lett. 560, L111.

Holder, G. P., and J. E. Carlstrom, 2001, Astroph. J. 558, 515.

Horner, D. J., M. E. Donahue, and G. M. Voit, 2004, in preparation .

Horner, D. J., R. F. Mushotzky, and C. A. Scharf, 1999, Astroph. J. 520, 78.

Hu, E. M., L. L. Cowie, and Z. Wang, 1985, Astroph. J. Suppl. 59, 447.

Hu, W., 2003, Phys. Rev. D 67, 081304.

Hu, W., and A. V. Kravtsov, 2003, Astroph. J. 584, 702.

Huterer, D., and M. White, 2002, Astroph. J. Lett. 578, L95.

Ikebe, Y., T. H. Reiprich, H. Böhringer, Y. Tanaka, and T. Kitayama, 2002, Astron. Astroph. 383, 773.

Inoue, S., and S. Sasaki, 2001, Astroph. J. 562, 618.

Irwin, J. A., and J. N. Bregman, 2000, Astroph. J. 538, 543.

Jarvis, M., G. M. Bernstein, P. Fischer, D. Smith, B. Jain, J. A. Tyson, and D. Wittman, 2003, Astron. J. 125, 1014.

Jenkins, A., C. S. Frenk, F. R. Pearce, P. A. Thomas, J. M. Colberg, S. D. M. White, H. M. P. Couchman, J. A. Peacock, G. Efstathiou, and A. H. Nelson, 1998, Astroph. J. 499, 20.

Jenkins, A., C. S. Frenk, S. D. M. White, J. M. Colberg, S. Cole, A. E. Evrard, H. M. P. Couchman, and N. Yoshida, 2001, Mot. Not. Roy. Ast. Soc. 321, 372.

Jing, Y. P., 2000, Astroph. J. 535, 30.

Johnstone, R. M., A. C. Fabian, and P. E. J. Nulsen, 1987, Mot. Not. Roy. Ast. Soc. 224, 75. 
Jones, C., and W. Forman, 1984, Astroph. J. 276, 38.

Jubelgas, M., V. Springel, and K. Dolag, 2004, Mot. Not. Roy. Ast. Soc. 351, 423.

Kaiser, C. R., and J. Binney, 2003, Mot. Not. Roy. Ast. Soc. 338, 837.

Kaiser, N., 1984, Astroph. J. Lett. 284, L9.

Kaiser, N., 1986, Mot. Not. Roy. Ast. Soc. 222, 323.

Kaiser, N., 1987, Mot. Not. Roy. Ast. Soc. 227, 1.

Kaiser, N., 1991, Astroph. J. 383, 104.

Kaiser, N., and G. Squires, 1993, Astroph. J. 404, 441.

Kay, S. T., 2004, Mot. Not. Roy. Ast. Soc. 347, L13.

Kay, S. T., P. A. Thomas, and T. Theuns, 2003, Mot. Not. Roy. Ast. Soc. 343, 608.

Kellogg, E., H. Gursky, H. Tananbaum, R. Giacconi, and K. Pounds, 1972, Astroph. J. Lett. 174, L65+.

Kent, S. M., and J. E. Gunn, 1982, Astron. J. 87, 945.

Kim, W.-T., and R. Narayan, 2003, Astrophys. J. 596, L139.

King, I., 1962, Astron. J. 67, 471.

Klypin, A. A., and A. I. Kopylov, 1983, Soviet Astronomy Letters 9, 41.

Knight, P. A., and T. J. Ponman, 1997, Mot. Not. Roy. Ast. Soc. 289, 955.

Kochanek, C. S., M. White, J. Huchra, L. Macri, T. H. Jarrett, S. E. Schneider, and J. Mader, 2003, Astroph. J. 585, 161.

Komatsu, E., and U. Seljak, 2001, Mot. Not. Roy. Ast. Soc. 327, 1353.

Kravtsov, A. V., and G. Yepes, 2000, Mot. Not. Roy. Ast. Soc. 318, 227.

Kriss, G. A., D. F. Cioffi, and C. R. Canizares, 1983, Astroph. J. 272, 439.

Kuo, C. L., P. A. R. Ade, J. J. Bock, C. Cantalupo, M. D. Daub, J. Goldstein, W. L. Holzapfel, A. E. Lange, M. Lueker, M. Newcomb, J. B. Peterson, J. Ruhl, et al., 2004, Astroph. J. 600, 32.

Lacey, C., and S. Cole, 1993, Mot. Not. Roy. Ast. Soc. 262, 627.

Lahav, O., M. J. Rees, P. B. Lilje, and J. R. Primack, 1991, Mot. Not. Roy. Ast. Soc. 251, 128.

Landau, L. D., and E. M. Lifshitz, 1959, Fluid mechanics (Course of theoretical physics, Oxford: Pergamon Press, 1959).

Larson, R. B., and H. L. Dinerstein, 1975, Proc. Ast. Soc. Pacific 87, 911.

Levine, E. S., A. E. Schulz, and M. White, 2002, Astroph. J. $\mathbf{5 7 7}, 569$.

Lewis, A. D., D. A. Buote, and J. T. Stocke, 2003, Astroph. J. 586, 135.

Lewis, A. D., E. Ellingson, S. L. Morris, and R. G. Carlberg, 1999, Astroph. J. 517, 587.

Lewis, G. F., A. Babul, N. Katz, T. Quinn, L. Hernquist, and D. H. Weinberg, 2000, Astroph. J. 536, 623.

Limber, D. N., and W. G. Mathews, 1960, Astroph. J. 132, 286.

Lin, Y., J. J. Mohr, and S. A. Stanford, 2003, Astroph. J. 591, 749 .

Lloyd-Davies, E. J., T. J. Ponman, and D. B. Cannon, 2000, Mot. Not. Roy. Ast. Soc. 315, 689.

Loewenstein, M., 2001, Astroph. J. 557, 573.

Loewenstein, M., and R. F. Mushotzky, 1996, Astroph. J. 466, 695.

Longair, M. S., and M. Seldner, 1979, Mot. Not. Roy. Ast. Soc. 189, 433.

Lubin, L. M., and N. A. Bahcall, 1993, Astroph. J. Lett. 415,
L17.

Lubin, L. M., J. S. Mulchaey, and M. Postman, 2004, Astroph. J. Lett. 601, L9.

Lumb, D. H., et al., 2003, eprint astro-ph/0311344.

Lumsden, S. L., R. C. Nichol, C. A. Collins, and L. Guzzo, 1992, Mot. Not. Roy. Ast. Soc. 258, 1.

Luppino, G. A., and I. M. Gioia, 1995, Astroph. J. Lett. 445, L77.

Lynden-Bell, D., 1967, Mot. Not. Roy. Ast. Soc. 136, 101.

Mac Low, M., and A. Ferrara, 1999, Astroph. J. 513, 142.

Magorrian, J., S. Tremaine, D. Richstone, R. Bender, G. Bower, A. Dressler, S. M. Faber, K. Gebhardt, R. Green, C. Grillmair, J. Kormendy, and T. Lauer, 1998, Astron. J. 115, 2285.

Majumdar, S., and J. J. Mohr, 2003, Astroph. J. 585, 603.

Majumdar, S., and J. J. Mohr, 2004, eprint astro-ph/0305341.

Malyshkin, L., 2001, Astroph. J. 554, 561.

Malyshkin, L., and R. Kulsrud, 2001, Astroph. J. 549, 402.

Markevitch, M., 1998, Astroph. J. 504, 27.

Markevitch, M., W. R. Forman, C. L. Sarazin, and A. Vikhlinin, 1998, Astroph. J. 503, 77.

Martin, C. L., 1999, Astroph. J. 513, 156.

Mather, J. C., E. S. Cheng, R. E. Eplee, R. B. Isaacman, S. S. Meyer, R. A. Shafer, R. Weiss, E. L. Wright, C. L. Bennett, N. W. Boggess, E. Dwek, S. Gulkis, et al., 1990, Astroph. J. Lett. 354, L37.

Mathews, W. G., and J. N. Bregman, 1978, Astroph. J. 224, 308.

Mathiesen, B. F., and A. E. Evrard, 2001, Astroph. J. 546, 100.

Matteucci, F., and B. K. Gibson, 1995, Astron. Astroph. 304, 11.

Mazzotta, P., E. Rasia, L. Moscardini, and G. Tormen, 2004, eprint astro-ph/0404425.

McNamara, B. R., and W. Jaffe, 1994, Astron. Astroph. 281, 673.

McNamara, B. R., and R. W. O'Connell, 1989, Astron. J. 98, 2018.

McNamara, B. R., M. Wise, P. E. J. Nulsen, L. P. David, C. L. Sarazin, M. Bautz, M. Markevitch, A. Vikhlinin, W. R. Forman, C. Jones, and D. E. Harris, 2000, Astroph. J. Lett. 534, L135.

McNamara, B. R., M. W. Wise, and S. S. Murray, 2004, Astroph. J. 601, 173.

Mellier, Y., 1999, Ann. Rev. Astron. Astroph. 37, 127.

Messier, C., 1784, Connaissance des Temps (Paris).

Metzler, C. A., M. White, and C. Loken, 2001, Astroph. J. $\mathbf{5 4 7}, 560$.

Metzler, C. A., M. White, M. Norman, and C. Loken, 1999, Astroph. J. Lett. 520, L9.

Mo, H. J., and S. D. M. White, 1996, Mot. Not. Roy. Ast. Soc. 282, 347.

Mohr, J. J., B. Mathiesen, and A. E. Evrard, 1999, Astroph. J. 517, 627.

Mohr, J. J., E. D. Reese, E. Ellingson, A. D. Lewis, and A. E. Evrard, 2000, Astroph. J. 544, 109.

Mota, D. F., and C. van de Bruck, 2004, eprint astro$\mathrm{ph} / 0401504$.

Muanwong, O., P. A. Thomas, S. T. Kay, and F. R. Pearce, 2002, Mot. Not. Roy. Ast. Soc. 336, 527.

Muanwong, O., P. A. Thomas, S. T. Kay, F. R. Pearce, and H. M. P. Couchman, 2001, Astroph. J. Lett. 552, L27.

Mulchaey, J. S., A. Dressler, and A. Oemler (eds.), 2004, Carnegie Astrophysics Series, Vol. 3: Clusters of Galax- 
ies: Probes of Cosmological Structure and Galaxy Evolution (Cambridge: Cambridge University Press).

Mullis, C. R., et al., 2004, eprint astro-ph/0401605.

Mushotzky, R. F., 1984, Physica Scripta Volume T 7, 157.

Mushotzky, R. F., 2004, in Carnegie Astrophysics Series, Vol. 3: Clusters of Galaxies: Probes of Cosmological Structure and Galaxy Evolution, edited by J. S. Mulchaey, A. Dressler, and A. Oemler, p. in press.

Mushotzky, R. F., and C. A. Scharf, 1997, Astroph. J. Lett. 482, L13+.

Narayan, R., and M. V. Medvedev, 2001, Astroph. J. Lett. 562, L129.

Nath, B. B., and S. Roychowdhury, 2002, Mot. Not. Roy. Ast. Soc. 333, 145.

Navarro, J. F., C. S. Frenk, and S. D. M. White, 1995, Mot. Not. Roy. Ast. Soc. 275, 720.

Navarro, J. F., C. S. Frenk, and S. D. M. White, 1997, Astroph. J. 490, 493.

Navarro, J. F., and S. D. M. White, 1993, Mot. Not. Roy. Ast. Soc. 265, 271.

Navarro, J. F., et al., 2003, eprint astro-ph/0311231.

Neumann, D. M., and M. Arnaud, 2001, Astron. Astroph. 373, L33.

Nevalainen, J., M. Markevitch, and W. Forman, 2000, Astroph. J. 536, 73.

Nichol, R. C., 2004, in Carnegie Astrophysics Series, Vol. 3: Clusters of Galaxies: Probes of Cosmological Structure and Galaxy Evolution, edited by J. S. Mulchaey, A. Dressler, and A. Oemler, p. in press.

Novicki, M. C., M. Sornig, and J. P. Henry, 2002, Astron. J. 124, 2413.

O'Connell, R. W., and B. R. McNamara, 1989, Astron. J. 98, 180.

O'Dea, C. P., S. A. Baum, P. R. Maloney, L. J. Tacconi, and W. B. Sparks, 1994, Astroph. J. 422, 467.

O’Dea, C. P., H. E. Payne, and D. Kocevski, 1998, Astron. J. 116, 623 .

Oh, S. P., and A. J. Benson, 2003, Mot. Not. Roy. Ast. Soc. 342, 664.

Osmond, J. P. F., and T. J. Ponman, 2004, Mot. Not. Roy. Ast. Soc. 350, 1511.

Oukbir, J., and A. Blanchard, 1992, Astron. Astroph. 262, L21.

Peacock, J. A., 1999, Cosmological Physics (Cosmological physics. Publisher: Cambridge, UK: Cambridge University Press, 1999. ISBN: 0521422701).

Peacock, J. A., and S. J. Dodds, 1994, Mot. Not. Roy. Ast. Soc. 267, 1020.

Pearce, F. R., P. A. Thomas, H. M. P. Couchman, and A. C. Edge, 2000, Mot. Not. Roy. Ast. Soc. 317, 1029.

Pearson, T. J., B. S. Mason, A. C. S. Readhead, M. C. Shepherd, J. L. Sievers, P. S. Udomprasert, J. K. Cartwright, A. J. Farmer, S. Padin, S. T. Myers, J. R. Bond, C. R. Contaldi, et al., 2003, Astroph. J. 591, 556.

Peebles, P. J., and B. Ratra, 2003, Reviews of Modern Physics 75, 559.

Peebles, P. J. E., 1993, Principles of physical cosmology (Princeton Series in Physics, Princeton, NJ: Princeton University Press, —c1993).

Peebles, P. J. E., R. A. Daly, and R. Juszkiewicz, 1989, Astroph. J. 347, 563.

Peebles, P. J. E., and J. T. Yu, 1970, Astroph. J. 162, 815.

Pen, U., 1997, New Astronomy 2, 309.

Penton, S. V., J. T. Stocke, and J. M. Shull, 2002, Astroph.
J. 565, 720 .

Perlmutter, S., G. Aldering, G. Goldhaber, R. A. Knop, P. Nugent, P. G. Castro, S. Deustua, S. Fabbro, A. Goobar, D. E. Groom, I. M. Hook, A. G. Kim, et al., 1999, Astroph. J. 517, 565 .

Peterson, J. R., S. M. Kahn, F. B. S. Paerels, J. S. Kaastra, T. Tamura, J. A. M. Bleeker, C. Ferrigno, and J. G. Jernigan, 2003, Astroph. J. 590, 207.

Peterson, J. R., F. B. S. Paerels, J. S. Kaastra, M. Arnaud, T. H. Reiprich, A. C. Fabian, R. F. Mushotzky, J. G. Jernigan, and I. Sakelliou, 2001, Astron. Astroph. 365, L104.

Pettini, M., A. E. Shapley, C. C. Steidel, J. Cuby, M. Dickinson, A. F. M. Moorwood, K. L. Adelberger, and M. Giavalisco, 2001, Astroph. J. 554, 981.

Pettini, M., C. C. Steidel, K. L. Adelberger, M. Dickinson, and M. Giavalisco, 2000, Astroph. J. 528, 96.

Pierpaoli, E., S. Borgani, D. Scott, and M. White, 2003, Mot. Not. Roy. Ast. Soc. 342, 163.

Pipino, A., F. Matteucci, S. Borgani, and A. Biviano, 2002, New Astronomy 7, 227.

Pizzolato, F., S. Molendi, S. Ghizzardi, and S. De Grandi, 2003, Astroph. J. 592, 62.

Ponman, T. J., D. B. Cannon, and J. F. Navarro, 1999, Nature (London) 397, 135.

Ponman, T. J., A. J. R. Sanderson, and A. Finoguenov, 2003, Mot. Not. Roy. Ast. Soc. 343, 331.

Portinari, L., A. Moretti, C. Chiosi, and J. Sommer-Larsen, 2003, eprint astro-ph/0312360.

Postman, M., J. P. Huchra, and M. J. Geller, 1992, Astroph. J. 384, 404.

Postman, M., T. R. Lauer, W. Oegerle, and M. Donahue, 2002, Astroph. J. 579, 93.

Postman, M., L. M. Lubin, J. E. Gunn, J. B. Oke, J. G. Hoessel, D. P. Schneider, and J. A. Christensen, 1996, Astron. J. 111, 615 .

Pratt, G. W., and M. Arnaud, 2002, Astron. Astroph. 394, 375.

Pratt, G. W., and M. Arnaud, 2003, Astron. Astroph. 408, 1.

Press, W. H., and P. Schechter, 1974, Astroph. J. 187, 425.

Randall, S. W., C. L. Sarazin, and P. M. Ricker, 2002, Astroph. J. 577, 579.

Rasia, E., G. Tormen, and L. Moscardini, 2003, eprint astroph/0309405.

Raymond, J. C., and B. W. Smith, 1977, Astroph. J. Suppl. 35, 419.

Regos, E., and M. J. Geller, 1989, Astron. J. 98, 755.

Reiprich, T. H., and H. Böhringer, 2002, Astroph. J. 567, 716.

Renzini, A., 1997, Astroph. J. 488, 35.

Renzini, A., 2004, in Carnegie Astrophysics Series, Vol. 3: Clusters of Galaxies: Probes of Cosmological Structure and Galaxy Evolution, edited by J. S. Mulchaey, A. Dressler, and A. Oemler, p. in press.

Ricker, P. M., and C. L. Sarazin, 2001, Astroph. J. 561, 621.

Riess, A. G., A. V. Filippenko, P. Challis, A. Clocchiatti, A. Diercks, P. M. Garnavich, R. L. Gilliland, C. J. Hogan, S. Jha, R. P. Kirshner, B. Leibundgut, M. M. Phillips, et al., 1998, Astron. J. 116, 1009.

Riess, A. G., L. Strolger, J. Tonry, S. Casertano, H. C. Ferguson, B. Mobasher, P. Challis, A. V. Filippenko, S. Jha, W. Li, R. Chornock, R. P. Kirshner, et al., 2004, Astroph. J. 607, 665 .

Rines, K., M. J. Geller, M. J. Kurtz, and A. Diaferio, 2003, 
Astron. J. 126, 2152.

Robinson, J., E. Gawiser, and J. Silk, 2000, Astroph. J. 532, 1.

Rood, H. J., T. L. Page, E. C. Kintner, and I. R. King, 1972, Astroph. J. 175, 627.

Rosati, P., S. Borgani, and C. Norman, 2002, Ann. Rev. Astron. Astroph. 40, 539.

Rosati, P., P. Tozzi, S. Ettori, V. Mainieri, R. Demarco, S. A. Stanford, C. Lidman, M. Nonino, S. Borgani, R. Della Ceca, P. Eisenhardt, B. P. Holden, et al., 2004, Astron. J. 127, 230.

Rosner, R., and W. H. Tucker, 1989, Astroph. J. 338, 761.

Ruszkowski, M., and M. C. Begelman, 2002, Astroph. J. 581, 223.

Sanders, R. H., 2003, Mot. Not. Roy. Ast. Soc. 342, 901.

Sanderson, A. J. R., T. J. Ponman, A. Finoguenov, E. J. Lloyd-Davies, and M. Markevitch, 2003, Mot. Not. Roy. Ast. Soc. 340, 989.

Sarazin, C. L., 1988, X-ray emission from clusters of galaxies (Cambridge Astrophysics Series, Cambridge: Cambridge University Press, 1988).

Sasaki, S., 1996, Pub. Ast. Soc. Japan 48, L119.

Scannapieco, E., and S. P. Oh, 2004, eprint astro-ph/0401087.

Schechter, P., 1976, Astroph. J. 203, 297.

Schirmer, M., T. Erben, P. Schneider, G. Pietrzynski, W. Gieren, S. Carpano, A. Micol, and F. Pierfederici, 2003, Astron. Astroph. 407, 869.

Schuecker, P., H. Böhringer, L. Guzzo, C. A. Collins, D. M. Neumann, S. Schindler, W. Voges, S. De Grandi, G. Chincarini, R. Cruddace, V. Müller, T. H. Reiprich, et al., 2001, Astron. Astroph. 368, 86.

Schuecker, P., R. R. Caldwell, H. Böhringer, C. A. Collins, L. Guzzo, and N. N. Weinberg, 2003, Astron. Astroph. 402, 53.

Seljak, U., 2002, Mot. Not. Roy. Ast. Soc. 337, 769.

Seljak, U., J. Burwell, and U. Pen, 2001, Phys. Rev. D 63, 063001.

Sheth, R. K., H. J. Mo, and G. Tormen, 2001, Mot. Not. Roy. Ast. Soc. 323, 1.

Sheth, R. K., and G. Tormen, 1999, Mot. Not. Roy. Ast. Soc. 308, 119 .

Shull, J. M., J. T. Stocke, and S. Penton, 1996, Astron. J. $111,72$.

Shull, J. M., J. Tumlinson, and M. L. Giroux, 2003, Astroph. J. Lett. 594, L107.

Smith, G. P., A. C. Edge, V. R. Eke, R. C. Nichol, I. Smail, and J. Kneib, 2003, Astroph. J. Lett. 590, L79.

Smith, S., 1936, Astroph. J. 83, 23.

Soker, N., 2003, Mot. Not. Roy. Ast. Soc. 342, 463.

Sparks, W. B., 1992, Astroph. J. 399, 66.

Spergel, D. N., L. Verde, H. V. Peiris, E. Komatsu, M. R. Nolta, C. L. Bennett, M. Halpern, G. Hinshaw, N. Jarosik, A. Kogut, M. Limon, S. S. Meyer, et al., 2003, Astroph. J. Suppl. 148, 175.

Spitzer, L., 1962, Physics of Fully Ionized Gases (Physics of Fully Ionized Gases, New York: Interscience (2nd edition), 1962).

Stanford, S. A., B. Holden, P. Rosati, P. Tozzi, S. Borgani, P. R. Eisenhardt, and H. Spinrad, 2001, Astroph. J. 552, 504.

Suginohara, T., and J. P. Ostriker, 1998, Astroph. J. 507, 16. Sugiyama, N., 1995, Astroph. J. Suppl. 100, 281.

Sunyaev, R. A., and Y. B. Zeldovich, 1970, Astroph. Sp. Sci. 7,3 .
Sunyaev, R. A., and Y. B. Zeldovich, 1972, Comments on Astrophysics 4, 173.

Sutherland, R. S., and M. A. Dopita, 1993, Astroph. J. Suppl. 88, 253.

Szalay, A. S., B. Jain, T. Matsubara, R. Scranton, M. S. Vogeley, A. Connolly, S. Dodelson, D. Eisenstein, J. A. Frieman, J. E. Gunn, L. Hui, D. Johnston, et al., 2003, Astroph. J. 591, 1.

The, L. S., and S. D. M. White, 1986, Astron. J. 92, 1248.

Thomas, P. A., O. Muanwong, F. R. Pearce, H. M. P. Couchman, A. C. Edge, A. Jenkins, and L. Onuora, 2001, Mot. Not. Roy. Ast. Soc. 324, 450.

Tornatore, L., S. Borgani, V. Springel, F. Matteucci, N. Menci, and G. Murante, 2003, Mot. Not. Roy. Ast. Soc. 342, 1025.

Tozzi, P., and C. Norman, 2001, Astroph. J. 546, 63.

Tozzi, P., P. Rosati, S. Ettori, S. Borgani, V. Mainieri, and C. Norman, 2003, Astroph. J. 593, 705.

Tran, K. H., D. D. Kelson, P. van Dokkum, M. Franx, G. D. Illingworth, and D. Magee, 1999, Astroph. J. 522, 39.

Tripp, T. M., B. D. Savage, and E. B. Jenkins, 2000, Astroph. J. Lett. 534, L1.

Tucker, W. H., and R. Rosner, 1983, Astroph. J. 267, 547.

Turner, M. S., and M. White, 1997, Phys. Rev. D 56, 4439.

Tyson, J. A., R. A. Wenk, and F. Valdes, 1990, Astroph. J. Lett. 349, L1.

Valageas, P., and J. Silk, 1999, Astron. Astroph. 350, 725.

Valdarnini, R., 2003, Mot. Not. Roy. Ast. Soc. 339, 1117.

van Haarlem, M. P., C. S. Frenk, and S. D. M. White, 1997, Mot. Not. Roy. Ast. Soc. 287, 817.

Viana, P. T. P., and A. R. Liddle, 1996, Mot. Not. Roy. Ast. Soc. 281, 323.

Viana, P. T. P., and A. R. Liddle, 1999, Mot. Not. Roy. Ast. Soc. 303, 535.

Vikhlinin, A., W. Forman, and C. Jones, 1999, Astroph. J. $\mathbf{5 2 5}, 47$.

Vikhlinin, A., L. VanSpeybroeck, M. Markevitch, W. R. Forman, and L. Grego, 2002, Astroph. J. Lett. 578, L107.

Vikhlinin, A., A. Voevodkin, C. R. Mullis, L. VanSpeybroeck, H. Quintana, B. R. McNamara, I. Gioia, A. Hornstrup, J. P. Henry, W. R. Forman, and C. Jones, 2003, Astroph. J. 590, 15.

Voevodkin, A., and A. Vikhlinin, 2004, Astroph. J. 601, 610.

Voigt, L. M., and A. C. Fabian, 2004, Mot. Not. Roy. Ast. Soc. 347, 1130.

Voit, G. M., 1994, Astroph. J. Lett. 432, L19.

Voit, G. M., 1996, Astroph. J. 465, 548.

Voit, G. M., 2000, Astroph. J. 543, 113.

Voit, G. M., M. L. Balogh, R. G. Bower, C. G. Lacey, and G. L. Bryan, 2003, Astroph. J. 593, 272.

Voit, G. M., and G. L. Bryan, 2001, Nature (London) 414, 425.

Voit, G. M., G. L. Bryan, M. L. Balogh, and R. G. Bower, 2002, Astrophys. J. 576, 601.

Voit, G. M., and M. Donahue, 1995, Astroph. J. 452, 164.

Voit, G. M., and M. Donahue, 1997, Astroph. J. 486, 242.

Voit, G. M., and M. Donahue, 1998, Astroph. J. Lett. 500, L111+.

Voit, G. M., A. E. Evrard, and G. L. Bryan, 2001, Astroph. J. Lett. 548, L123.

Voit, G. M., S. T. Kay, and G. L. Bryan, 2004, in preparation

Voit, G. M., and T. J. Ponman, 2003, Astroph. J. Lett. 594, L75. 
Wang, L., and P. J. Steinhardt, 1998, Astroph. J. 508, 483.

Waugh, M., M. J. Drinkwater, R. L. Webster, L. StaveleySmith, V. A. Kilborn, D. G. Barnes, R. Bhathal, W. J. G. de Blok, P. J. Boyce, M. J. Disney, R. D. Ekers, K. C. Freeman, et al., 2002, Mot. Not. Roy. Ast. Soc. 337, 641.

Weinberg, N. N., and M. Kamionkowski, 2003, Mot. Not. Roy. Ast. Soc. 341, 251.

Weller, J., R. A. Battye, and R. Kneissl, 2002, Physical Review Letters 88, 231301.

Weymann, R., 1965, Phys. Fluids 8, 2112.

Weymann, R., 1966, Astroph. J. 145, 560.

White, D. A., C. Jones, and W. Forman, 1997, Mot. Not. Roy. Ast. Soc. 292, 419.

White, M., 2001, Astron. Astroph. 367, 27.

White, M., G. Gelmini, and J. Silk, 1995, Phys. Rev. D 51, 2669.

White, M., L. Hernquist, and V. Springel, 2002, Astroph. J. $\mathbf{5 7 9}, 16$.

White, S. D. M., and C. S. Frenk, 1991, Astroph. J. 379, 52.

White, S. D. M., C. S. Frenk, M. Davis, and G. Efstathiou, 1987, Astroph. J. 313, 505.
White, S. D. M., J. F. Navarro, A. E. Evrard, and C. S. Frenk, 1993, Nature (London) $\mathbf{3 6 6}, 429$.

White, S. D. M., and M. J. Rees, 1978, Mot. Not. Roy. Ast. Soc. 183, 341.

Wittman, D., V. E. Margoniner, J. A. Tyson, J. G. Cohen, A. C. Becker, and I. P. Dell'Antonio, 2003, Astroph. J. 597, 218.

Wittman, D., J. A. Tyson, V. E. Margoniner, J. G. Cohen, and I. P. Dell'Antonio, 2001, Astroph. J. Lett. 557, L89.

Wu, K. K. S., A. C. Fabian, and P. E. J. Nulsen, 2001, Mot. Not. Roy. Ast. Soc. 324, 95.

Wu, X., and Y. Xue, 2002, Astroph. J. Lett. 572, L19.

Xue, Y., and X. Wu, 2000, Astroph. J. 538, 65.

Yee, H. K. C., and E. Ellingson, 2003, Astroph. J. 585, 215.

Yee, H. K. C., and O. López-Cruz, 1999, Astron. J. 117, 1985.

Zaroubi, S., G. Squires, G. de Gasperis, A. E. Evrard, Y. Hoffman, and J. Silk, 2001, Astroph. J. 561, 600.

Zeldovich, Y. B., 1972, Mot. Not. Roy. Ast. Soc. 160, 1P.

Zwicky, F., 1933, Helv. physica acta 6, 110.

Zwicky, F., 1937, Astroph. J. 86, 217. 